\title{
McMaster University
}

\section{DigitalCommons@McMaster}

Open Access Dissertations and Theses

Open Dissertations and Theses

4-1-2012

\section{Detecting Radiation Pressure in Waveguides Using Microelectromechanical Resonators}

Christopher R P Pope

McMaster University, chris.pope@usask.ca

Follow this and additional works at: http://digitalcommons.mcmaster.ca/opendissertations

Part of the Nanoscience and Nanotechnology Commons

\section{Recommended Citation}

Pope, Christopher R P, "Detecting Radiation Pressure in Waveguides Using Microelectromechanical Resonators" (2012). Open Access Dissertations and Theses. Paper 6592.

This Thesis is brought to you for free and open access by the Open Dissertations and Theses at DigitalCommons@McMaster. It has been accepted for inclusion in Open Access Dissertations and Theses by an authorized administrator of DigitalCommons@McMaster. For more information, please contactscom@mcmaster.ca. 
RADIATION PRESSURE IN WAVEGUIDE RESONATORS 


\title{
DETECTING RADIATION PRESSURE IN WAVEGUIDES USING MICROELECTROMECHANICAL RESONATORS
}

By CHRISTOPHER POPE, B.Sc

A Thesis Submitted to the School of Graduate Studies in Partial Fulfilment of the Requirements for the Degree Master of Applied Science

\author{
McMaster University
}

(C) Copyright by Christopher Pope, December 2011 
McMaster University MASTER OF APPLIED SCIENCE (2011) Hamilton, Ontario (Engineering Physics)

TITLE: Detecting Radiation Pressure in Waveguides Using Microelectromechanical Resonators

AUTHOR: Christopher Pope, B.Sc. (University of Saskatchewan) SUPERVISOR: Dr. Rafael N. Kleiman NUMBER OF PAGES: $\mathrm{x}, 84$ 


\section{Abstract}

The phenomenon of radiation pressure has fascinated scientists since it was first proposed by Maxwell in the late $19^{\text {th }}$ century. Numerous experiments involving optical forces have been carried out, however the optical force acting on a curved waveguide does not appear to have been previously investigated. An experiment to measure the force acting on a waveguide due to the optical power it contains is proposed here. This experiment takes advantage of the sensitivity of MicroElectroMechanical Systems (MEMS) and the performance of silicon integrated optics in a single hybrid device.

Devices are fabricated from silicon-on-insulator (SOI) wafers using conventional micromachining techniques. Anisotropic alkali etches are used to

produce smooth vertical side-walls for a mechanical structure and a rib waveguide. An analysis of the electrical systems and measurement techniques is provided. Using these techniques, the resonant operation of the devices is demonstrated by means of capacitive actuation and sensing. The application of this system to the measurement of radiation pressure is discussed. 


\section{Acknowledgements}

I wish to thank my supervisor, Dr. Rafael N. Kleiman for inspiring this project, and for his helpful insight and guidance throughout this project. I am grateful to Abhishaik Rampal for the extensive advice, assistance and support provided during this endeavor. I am thankful for the training and assistance provided by Dr. Zhilin Peng and Doris Stevanovic from the Centre for Emerging Device Technologies, which made fabrication possible. I wish thank my colleages Dr. Sri Priya Sundararajan, Matthew Minnick, Sarah Selesnic and Nikolaus Jewell for helpful discussions and advice during the course of the project. Finally, I would like to thank my parents for their continued support in this endeavor. 


\section{Contents}

List of Figures. . . . . . . . . . . . . . . . . . . vii

List of Tables . . . . . . . . . . . . . . . . . . . . . . viii

Notation ........................ . . ix

Abbreviations ................... . . $\mathrm{x}$

\begin{tabular}{lll}
\hline & Introduction & 1
\end{tabular}

1.1 Radiation Pressure . . . . . . . . . . . . . . . . . . . . 1

1.2 MEMS . . . . . . . . . . . . . . . . . . . . . . 2

1.3 Thesis Overview . . . . . . . . . . . . . . . . . . . 3

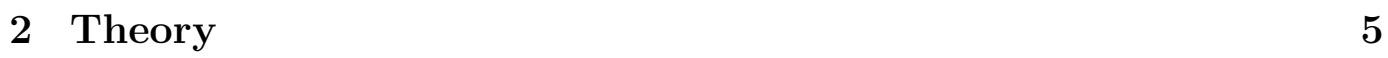

2.1 Abraham and Minkowski . . . . . . . . . . . . . . . . 5

2.2 Optical Forces . . . . . . . . . . . . . . . . . . . . 13

2.3 Elasticity . . . . . . . . . . . . . . . . . . . . . 18

2.4 Waveguides . . . . . . . . . . . . . . . . . . . . . . 23

2.5 Electromechanical systems . . . . . . . . . . . . . . . 25

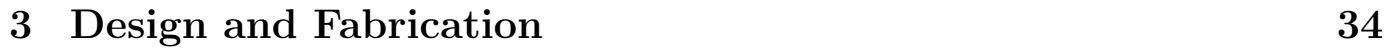

3.1 Fabrication Techniques . . . . . . . . . . . . . . . . . . 34

3.2 Device Design . . . . . . . . . . . . . . . . . . . . . . 36

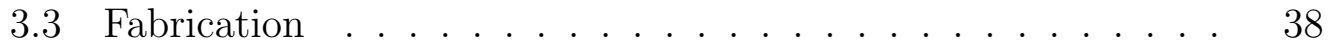

3.4 Fabrication challenges . . . . . . . . . . . . . . . 44

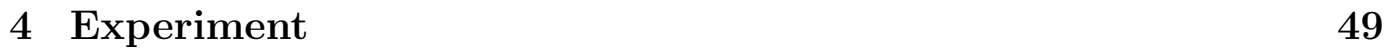

$4.1 \quad$ Electrical Experiment . . . . . . . . . . . . . . . . . . . . . . . 49

4.2 Resonance Measurements . . . . . . . . . . . . . . . . . . . . . 59

4.3 Effective Spring Constant . . . . . . . . . . . . . . . . . . . 64

4.4 Optical Experiment . . . . . . . . . . . . . . . . . . 71

$\begin{array}{lll}5 & \text { Conclusion } & 73\end{array}$

5.1 Summary . . . . . . . . . . . . . . . . . . . . 73

5.2 Future Work . . . . . . . . . . . . . . . . . . . . . . . 74

\begin{tabular}{ll}
\hline References & 76
\end{tabular} 


\section{List of Figures}

1.1 Fabricated device . . . . . . . . . . . . . . . . . 4

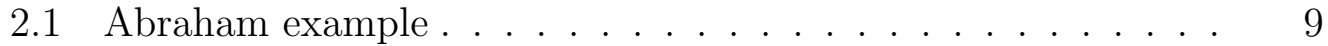

2.2 Minkowski example . . . . . . . . . . . . . . . . . . . . . . . . 10

2.3 Photon interaction $\ldots \ldots \ldots \ldots \ldots \ldots$

2.4 Beam bending . . . . . . . . . . . . . . . . . . . . . . . . . . . . . . . 21

$2.5 \quad$ Mode shape of a bridge . . . . . . . . . . . . . . . . . . . . . . . 23

$2.6 \quad$ Mode shape of a cantilever . . . . . . . . . . . . . . . . . . . . 24

2.7 SOI rib waveguide $\ldots \ldots \ldots \ldots \ldots$

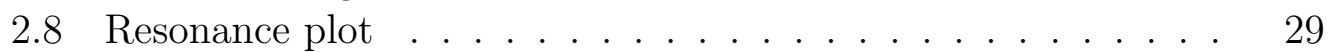

2.9 Pull-in voltage $\ldots \ldots \ldots \ldots \ldots$

$3.1 \quad$ Anisotropically etched bridge $\ldots \ldots \ldots \ldots$. . . . . . . 36

3.2 Rendering of device . . . . . . . . . . . . . . . . . . . . . . 37

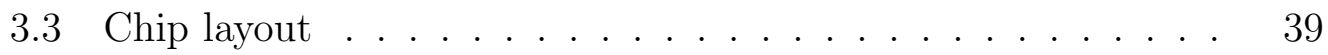

3.4 Photolithography masks . . . . . . . . . . . . . . . . . . 40

3.5 Process cross-sections . . . . . . . . . . . . . . . . . . . . . . . 41

3.6 Fabricated device . . . . . . . . . . . . . . . . . . . . . . . . . 43

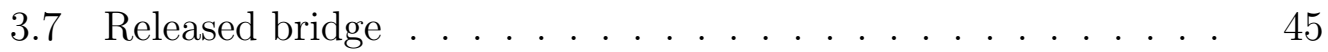

$3.8 \quad$ Cleaved edge $\ldots \ldots \ldots \ldots \ldots$

3.9 Curved bridge . . . . . . . . . . . . . . . . . . . . . . 47

$4.1 \quad$ Electrode schematic . . . . . . . . . . . . . . . . . . . . . 50

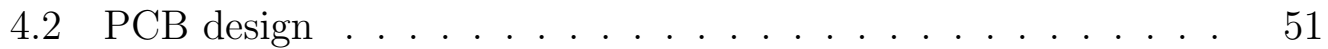

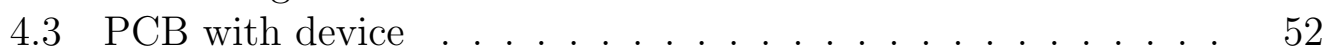

4.4 Three-terminal schematic . . . . . . . . . . . . . . . . . 53

4.5 Measurement schematic . . . . . . . . . . . . . . . . 56

4.6 System block diagram . . . . . . . . . . . . . . . . . . . . 5

4.7 Destroyed device $\ldots \ldots \ldots \ldots \ldots$

$4.8 \quad$ Resonance plot $-1000 \mu \mathrm{m}$ device $\ldots \ldots \ldots \ldots$

4.9 Resonance plot $-600 \mu \mathrm{m}$ device $\ldots \ldots \ldots$. . . . . . 60

4.10 Resonance stability $\ldots \ldots \ldots \ldots$

4.11 Effect of balancing . . . . . . . . . . . . . . . . . . 63

4.12 Overdriven response $\ldots \ldots \ldots$ 
4.13 Resonance shift $-600 \mu \mathrm{m}$. . . . . . . . . . . . . . . 65

4.14 Frequency vs Bias $-600 \mu \mathrm{m}$. . . . . . . . . . . . . . . . . 66

4.15 Response vs Bias $-600 \mu \mathrm{m}$. . . . . . . . . . . . . . . . . . . 67

4.16 Resonance shift $-1 \mathrm{~mm}$. . . . . . . . . . . . . . . . . 68

4.17 Frequency vs Bias $-1 \mathrm{~mm}$. . . . . . . . . . . . . . . 69

4.18 Response vs Bias $-1 \mathrm{~mm}$. . . . . . . . . . . . . . . . . . 70

4.19 Separate bias circuit . . . . . . . . . . . . . . . . . . . . . . . . 70

4.20 Optical experiment setup . . . . . . . . . . . . . . . . . 72 


\section{List of Tables}

2.1 Maxwell's Equations . . . . . . . . . . . . . . . . . . . . 7

2.2 Expressions for electromagnetic momentum. . . . . . . . . . . 8

3.1 Micromachining etches . . . . . . . . . . . . . . . . 35

4.1 Calculated device capacitances . . . . . . . . . . . . . . . . 54

4.2 Measured device capacitance . . . . . . . . . . . . . . . . . . 55

4.3 Measured and calculated resonant frequencies . . . . . . . . . 61 


\section{Notation}

c Speed of light in a vacuum

$\vec{D} \quad$ Dielectric field

$E \quad$ Young's modulus

$\vec{E} \quad$ Electric field

$f \quad$ Frequency, force density

$\vec{g}$ Momentum density

$h$ Planck's constant

$\hbar$ Reduced Planck's constant $h / 2 \pi$

$k$ Spring constant, stiffness

$\mathcal{P}$ Pressure

$P$ Power

$\epsilon \quad$ Dielectric constant, Strain

$\epsilon_{0} \quad$ Electric constant (permittivity of free space)

$\mu_{0} \quad$ Magnetic constant (permeability of free space)

$\lambda$ Wavelength

$\omega$ Angular frequency 


\title{
Abbreviations
}

\author{
AC Alternating Current \\ BHF Buffered HF \\ CVD Chemical Vapour Deposition \\ CPD Critical Point Drying \\ DIP Dual Inline Pin \\ DC Direct Current \\ DUT Device Under Test \\ EM Electromagnetic \\ HF Hydrogen Fluoride, Hydrofluoric Acid \\ IPA Isopropyl Alcohol \\ SNR Signal to Noise Ratio \\ SOI Silicon-on-Insulator \\ PCB Printed Circuit Board
}




\section{Chapter 1}

\section{Introduction}

\subsection{Radiation Pressure}

Radiation pressure has been a subject of interest since the pressure exerted by light on a surface was first calculated by Maxwell in 1873 [1, 2]. The first measurements of the radiation pressure force were made by Nichols and Hull [3, 4] and independently by Lebedew [5] in 1901 .

An interesting use of radiation pressure forces is in optical tweezers, first demonstrated by Ashkin in 1969 [6, 7]. Transparent particles can be trapped at the focus of a laser beam, as in a potential well. Light refracted by the particle experiences a change in momentum which generates an optical restoring force. These techniques have been extended from inert spheres [8] to living cells [9] and to the trapping and cooling of atoms [10, 11], opening up opportunities for new science [12].

Another potential application of radiation pressure is spacecraft propulsion in the form of solar sails [13]. A thin membrane with a large surface area can capture sufficient energy to accelerate a spacecraft while requiring no reaction mass. Solar sails have been employed in the Japanese IKAROS and NASA's NanoSail-D missions [14, 15], and further missions are planned. Solar sails are also popular topics in science fiction where they were popularized by stories such as Arthur C. Clarke's Sunjammer [16].

The momentum carried by a photon or electromagnetic wave propagating in free space is well understood, but the momentum of light in a medium has been the subject of debate. The first expression was proposed by Minkowski [17, 18] who calculated that the momentum density of an EM wave propagating in a dielectric medium should increase relative to its free-space value by a factor $n$ equal to the refractive index of the medium. Abraham [19, 20] calculated a different expression for the momentum density, suggesting that it should instead decrease by a factor of $n$. This is the origin of what is know as the Abraham-Minkowski controversy, which has been discussed by a 
number of authors for the last century. Recent works have claimed to resolve this controversy theoretically [21, 22, 23] and experimentally [24], though the experimental results are disputed [25, 26].

\subsection{MEMS}

Several terms, including microsystems and microelectromechanical systems (MEMS), are used to describe the growing number of microscale devices coupling mechanical structures to electrical, optical, thermal and other physical effects [27]. An early call to take advantage of the opportunities offered by miniturization was made by Richard Feynman in his classic 1959 talk There's Plenty of Room at the Bottom [28. Industrial applications beginning in the 1970s included pressure sensors, print heads, inertial sensors and optical devices with a variety of other possible applications under development [27, 29].

Although not usually classified as a MEMS device, and preceding the IC industry by several decades, the most common micromechanical device is probably the crystal oscillator. First developed around 1920 [30] they allowed for significantly better time-keeping than mechanical mechanisms. Significant effort has been invested in improving the technology due to the value of accurate frequency standards in radio receivers. In modern digital electronics crystal oscillators are commonly used to provide clock references for digital logic, timekeeping and wireless communication systems.

The first reported use of a silicon-based electromechanical system was the resonant gate transistor in 1965 [31, 32] which combines a conventional metaloxide-semiconductor (MOS) transistor with a moving beam which forms the gate. The original experiments demonstrated a band-pass behavior at the mechanical resonant frequency of the beam, foreshadowing later radio-frequency MEMS applications [33]. The integration with MOS circuitry and fabrication techniques demonstrated some of the advantages of silicon over other competing materials.

Silicon is a good material for MEMS applications due to material properties and synergies with integrated circuit (IC) processing methods [34]. Singlecrystal silicon is a nearly perfect Hookesian material with tensile strength and Young's modulus comparable to steel, allowing the construction of durable devices. Many manufacturing techniques developed for CMOS IC processing have been adapted to the production of microsystems including photolithography, wet and dry etching and chemical vapour deposition (CVD) [29]. The availability of processing equipment, raw materials and expertise reduces costs and makes silicon a more attractive material. The possibility of integrating circuitry with silicon-based mechanical devices is another advantage.

An early and ongoing area of interest has been the integration of optical and MEMS devices. Early examples included light modulators [35] and steerable 
mirrors [36]. One of the more wide-spread optical MEMS devices is the Digital Light Processor (DLP), used for video projection [37] and a variety of other applications. Recent examples have included attenuators based on moving waveguides [38] and optical switches [39, 40].

Optical actuation of microsystems has been an area of interest in recent years. An early example demonstrated the force of a laser beam on a MEMS mirror, and used this force to excite the mechanical resonance of the structure [41. Recent works have proposed the existence of optical forces due to evanescent fields from a dielectric waveguide [42]. This effect has been demonstrated in nanoscale silicon waveguides [43, 44] and in a whispering gallery resonator [45.

MEMS devices have been used in research environments to measure very small forces. One example is a force transducer used to measure the contraction force exerted by a single heart cell [46]. Forces in the $\mu \mathrm{N}$ range were successfully measured.

\subsection{Thesis Overview}

This project proposes to measure radiation pressure forces in a novel geometry by harnessing the flexibility of MEMS fabrication and sensing techniques. A section of a silicon waveguide is fabricated as a double-clamped beam which forms a mechanical resonator. As the beam bends the light propagating in the waveguide exerts a restoring force on the beam. This force is an optical stiffness which adds to the mechanical spring constant of the structure. When the mechanical structure is driven in resonance the optical stiffness can be detected as a change in resonance frequency with changing optical power. Using a resonant structure allows for an increased sensitivity by filtering out DC effects.

The design, fabrication and operation of devices which can carry out this experiment is described in this thesis. Devices are fabricated from SOI wafers by means of anisotropic etching, and the mechanical structures have been successfully released. An example of a fabricated device is shown in Figure 1.1. The techniques necessary for capacitive actuation and detection of the MEMS resonator are discussed. The resonant behavior of the device is investigated and an electrostatic softening of the structure is demonstrated by observing a decrease in the resonant frequency. The theory of optical-mechanical interaction is discussed, along with the proposed optical experiment. 


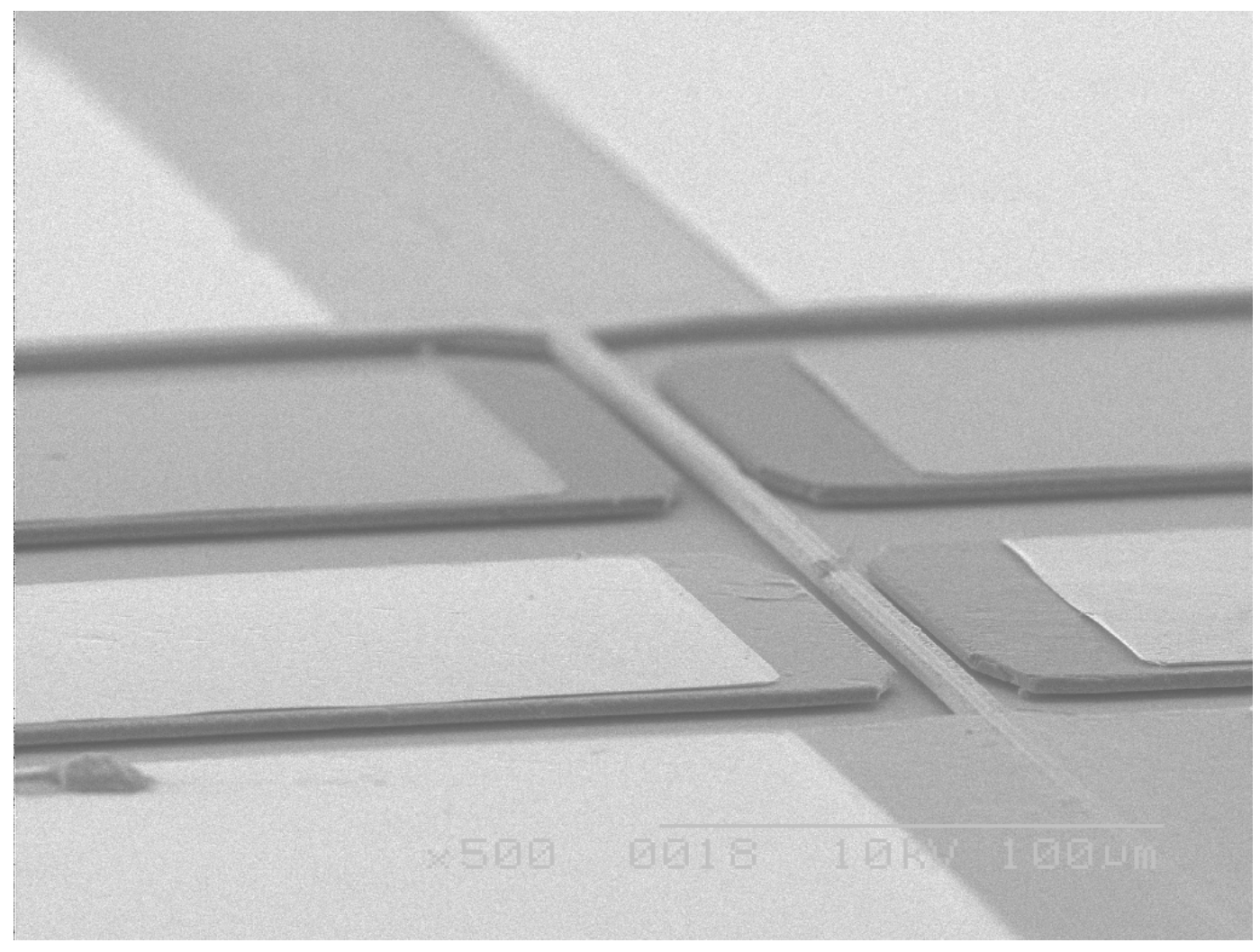

Figure 1.1: SEM micrograph of a fabricated device. The bridge length is 400 microns. 


\section{Chapter 2}

\section{Theory}

\subsection{Abraham and Minkowski}

\subsubsection{Energy and Momentum}

Kinetic energy and momentum are related classically by the expression

$$
E=\frac{p^{2}}{2 m}
$$

which is valid for slow-moving massive particles. Special relativity provides the alternate expression [47]

$$
E=c \sqrt{p^{2}+m^{2} c^{2}}
$$

which is valid for all particles, including photons where it reduces to

$$
E=p c
$$

or alternately

$$
p=\frac{E}{c}=\frac{h f}{c}
$$

The de Broglie wavelength of a particle is related to its momentum by

$$
\lambda=\frac{h}{p}
$$

This expressions is valid for both massive and massless particles. For a photon this reduces to

$$
\lambda=\frac{c}{f}
$$


While the above analysis is based on the particle picture of light it is equally valid to consider the case of a traveling electromagnetic wave. If the wave caries an energy flux $S$ (energy per unit time per unit area) in the direction of propagation then it has an energy density

$$
U=\frac{S}{c}
$$

in the steady state, where $c$ is the speed of propagation. Using the relation from (2.4) the momentum density of the wave is

$$
g=\frac{S}{c^{2}}
$$

This is the natural form when dealing with wave problems, such as light confined in a waveguide.

\subsubsection{Two Approaches}

Electromagnetic problems are typically analyzed through the use of Maxwell's equations in some form. Depending on the nature of the problem these equations can be constructed in a number of forms and popularly in two choices of field variables 48. The first choice is in terms of the electric field $\vec{E}$ and the magnetic field $\vec{B}, \overrightarrow{1}$ sometimes referred to as the microscopic fields. The physics described by Maxwell's equations in this form is universal and is the basis for understanding the electromagnetic behavior of matter. For this reason they are referred to as the microscopic fields.

In the presence of a medium which experiences polarization or magnetization it is sometimes convenient to abstract the material properties into simple constants. Maxwell's equations may then be constructed in terms of the dielectric field $\vec{D}$ and the magnetizing field $\vec{H}$, also called the macroscopic field variables. These fields depend only on the free charge and current respectively and therefore may be more convenient as it is the free parameters that are typically under experimental control $2^{2}$

The photon momentum given in Equation (2.4) is valid for the free-space or microscopic case. This corresponds to a momentum density

$$
\vec{g}=\frac{\vec{S}}{c^{2}}
$$

\footnotetext{
${ }^{1}$ The choice of names for the $\vec{B}$ and $\vec{H}$ fields varies, with some authors preferring to call $\vec{H}$ the magnetic field.

${ }^{2}$ In fact, it is more usual to control the electric potential $V$ rather than the free charge, making $\vec{E}$ and $\vec{H}$ the natural variables in some engineering applications.
} 


$$
\begin{aligned}
\nabla \cdot \vec{E} & =\frac{\rho}{\epsilon_{0}} & \nabla \cdot \vec{D} & =\rho_{f} \\
\nabla \times \vec{E} & =-\frac{d \vec{B}}{d t} & \nabla \times \vec{D} & =-\frac{d \vec{B}}{d t} \\
\nabla \cdot \vec{B} & =0 & \nabla \cdot \vec{B} & =0 \\
\nabla \times \vec{B} & =\mu_{0} \nabla \cdot \vec{J}+\mu_{0} \epsilon_{0} \frac{d \vec{E}}{d t} & \nabla \times \vec{H} & =\nabla \cdot \overrightarrow{J_{f}}+\frac{d \vec{D}}{d t}
\end{aligned}
$$

Table 2.1: Maxwell's equations, in both microscopic and macroscopic forms. [48]

where the Poynting vector $\vec{S}$ is the energy flux carried by the fields. 49. The Poynting vector is given by

$$
\vec{S}=\vec{E} \times \vec{H}
$$

For electromagnetic waves it is more convenient to use the definition

$$
\vec{S}=\frac{1}{2} \mathfrak{R e}\left[\vec{E} \times \vec{H}^{*}\right]
$$

which is known as the time-averaged Poynting vector, representing the average rate of energy transfer, or power, carried by the wave. A simpler form is the ratio of the power carried by a wave to the area that wave passes through

$$
S=\frac{P}{A}
$$

which is the form most useful when considering EM waves in a waveguide.

In the case of a photon traveling in a medium the situation is more interesting. Two forms for the photon momentum have been advanced, both supported by theory and experiment. The first formulation, proposed by Hermann Minkowski in 1908 [17, 18] suggests an increase of the momentum in a medium. The second form, proposed by Max Abraham in 1909 [19, 20] predicts a decrease in the momentum on entering a medium. In a dielectric medium with a refractive index $n$ these two forms differ by a factor of $n^{2}$. The expressions for electromagnetic momentum are summarized in Table 2.2. A number of arguments have been made by various authors for one form or the other. An example from each side is presented here, along with an overview of relevant experiments and the current state of the controversy. Extensive overviews of the literature can be found in references [50, 51, 21]. 
Masters Thesis - Christopher Pope McMaster University - Eng. Physics

\begin{tabular}{c|ccc} 
& Photon Momentum & Momentum Density & Interpretation \\
\hline Maxwell & $p=\frac{h f}{c}$ & $\vec{g}=\frac{\vec{S}}{c^{2}}$ & Free-space \\
Minkowski & $p=\frac{n h f}{c}$ & $\vec{g}=\frac{\vec{E} \times \vec{H}}{c^{2}}$ & Canonical \\
Abraham & $p=\frac{h f}{n c}$ & $\vec{g}=\vec{D} \times \vec{B}$ & Kinetic
\end{tabular}

Table 2.2: Summary of the three expressions for electromagnetic momentum, in both the single-photon and momentum density forms.

\subsubsection{Abraham}

Abraham's form for the electromagnetic momentum appears natural from a mechanical point of view. If the photon behaved like a massive particle then slowing as it entered a medium would naturally reduce its momentum. The argument presented here follows the example of Barnett and Loudon [21] who present a simplification of the work of Balazs [52].

The Abraham momentum may be derived by considering the case of a photon entering a transparent block, as illustrated in Figure 2.1. The block has mass $M$ and is initially at rest while the photon is moving with a velocity $c$ and an energy $h f$. Upon entering the block the photon slows to a speed $c / n$ and travels the block length $L$ in a time $n L / c$. Had the photon passed by the block it would have traveled a distance $n L$ in this time, a difference of $L(n-1)$. Relativity requires that the center of mass-energy of an isolated system experience uniform motion. Thus, the distance traveled by the center of mass-energy must be the same in both cases

$$
\begin{gathered}
0 M c^{2}+n L h f=\Delta x M c^{2}+L h f \\
\Delta x M c^{2}=(n-1) L h f \\
\Delta x=L(n-1) \frac{h f}{M c^{2}}
\end{gathered}
$$

requiring the block to be displaced by a distance $\Delta x 3^{3}$ Conservation of mo-

\footnotetext{
${ }^{3}$ This ignores the effect of the motion of the block on the motion of the photon which is valid for $\Delta x \ll L$ or $h f \ll M c^{2}$
} 
mentum then gives

$$
\begin{gathered}
\frac{h f}{c}=p_{A b r}+M v \\
p_{A b r}=\frac{h f}{c}-M\left[L(n-1) \frac{h f}{M c^{2}}\right] \frac{c}{n L} \\
p_{A b r}=\frac{h f}{n c}
\end{gathered}
$$

where $p_{A b r}$ is the photon momentum. Thus, the photon momentum is reduced upon entering the medium and transfers a fraction $(n-1) / n$ of its momentum to the medium.
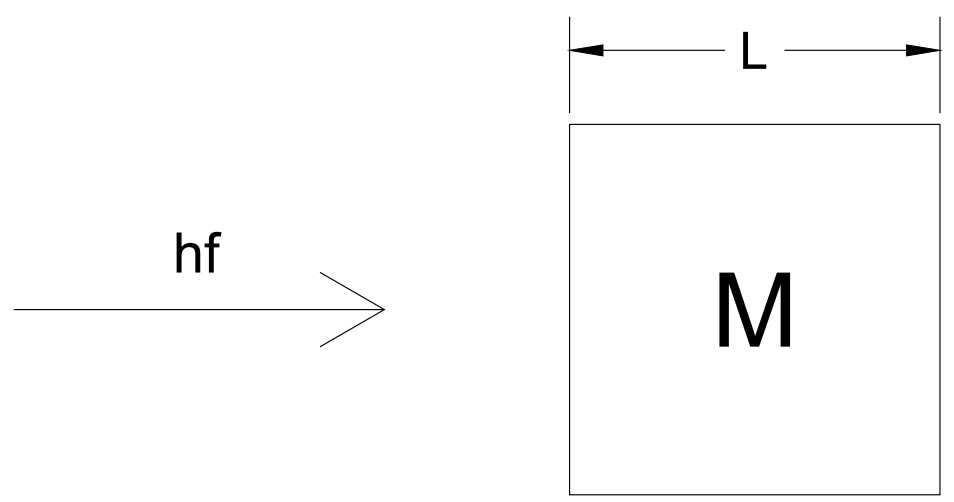

Figure 2.1: Diagram illustrating the argument for the Abraham form of the electromagnetic momentum based on the uniform motion of the center of massenergy.

\subsubsection{Minkowski}

Minkowski's momentum appears natural when one considers the de Broglie relation $p=h / \lambda$ relating momentum to wavelength. If a photon slows as it enters a medium then its wavelength must also shrink, given that its frequency is constant. An analytical approach based on this change in wavelength is given here, again following Barnett and Loudon[21] based on the work of Padgett [53].

A collimated light beam incident on a slit of width $\Delta x$ undergoes diffraction and spreads to an angle

$$
\theta=\frac{\lambda}{\Delta x}
$$


as shown in Figure 2.2. This angle can be approximated as the ratio of the axial to transverse momentum

$$
\theta \approx \frac{\Delta p_{x}}{p_{z}}
$$

for small divergences. In this case the axial momentum $p_{z}$ is approximately equal to the incident momentum $p_{0}$. The uncertainty principle

$$
\Delta x \Delta p \leq \hbar
$$

allows us to rewrite (2.21) as

$$
\theta=\frac{\hbar}{\Delta x p_{0}}
$$

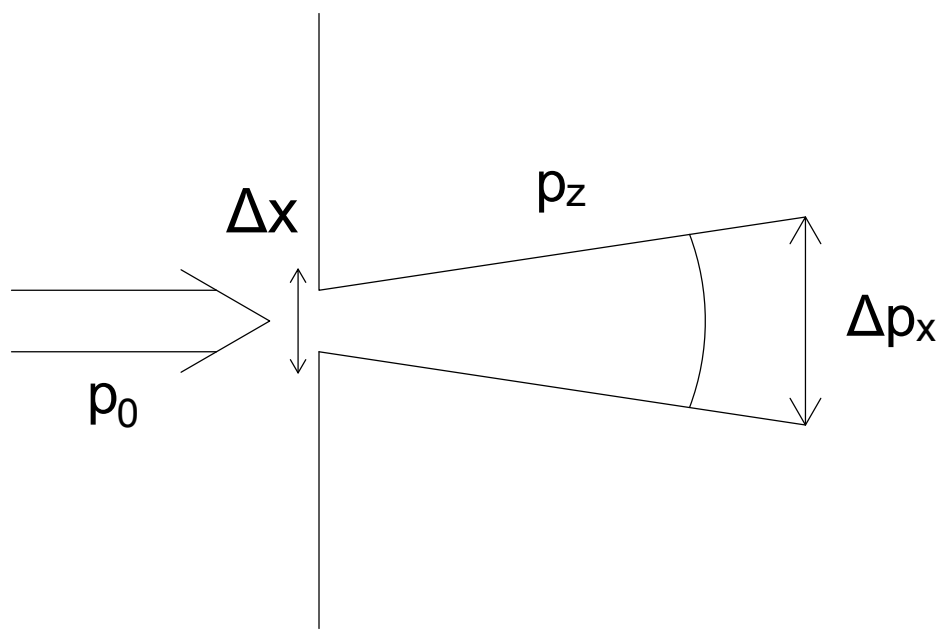

Figure 2.2: Diagram illustrating the argument for the Minkowski form of the electromagnetic momentum based on diffraction and the uncertainty principle.

If the entire experiment is then immersed in a dielectric liquid with refractive index $n$ the wavelength of the incident light is reduced by a factor of $n$. By Equation 2.20 the diffraction angle must also be reduced to

$$
\theta=\frac{\hbar}{\Delta x n p_{0}}
$$

which gives the Minkowski momentum as

$$
p_{\text {Min }}=\frac{n \hbar \omega}{c}
$$

Thus, the photon momentum increases in the medium, representing the additional momentum being carried by the polarization of the medium. 


\subsubsection{Selected experiments}

In addition to the work of Nichols and Hull in 1901 [3, 4, a number of other experiments have been performed both to measure radiation pressure and in an attempt to resolve the Abraham-Minkowski controversy. The key challenge in measuring radiation pressure is the magnitude of the force involved, as even one watt of optical power produces a force of only $3 \mathrm{nN}$ when absorbed. This places stringent requirements on experimental design, with the first work using a torsion balance under high vacuum. A second and closely related challenge is proving that the observed effect is actually due to radiation pressure and not some other mechanism, typically thermally driven. The Crooke's radiometer, now a popular novelty item, consists of rotating vanes, dark on one side and reflective on the other, contained in a partially evacuated bulb. While the radiometer was originally believed to derive its motion from radiation pressure forces, in actuality the vanes rotate due to thermal gradients. Unequal heating of the two sides of the vane causes air currents in an imperfect vacuum[54, a fact that can be readily proven simply by completely evacuating the bulb.4 Experimental design therefore must take into account thermal effects, typically by using high-reflectivity mirrors.

Photons also carry angular momentum of magnitude $h$, and a measurement of the angular momentum transfer required to change the polarization of a light beam was made in 1936 by Beth [55]. A light beam was passed through a quarter wave plate suspended from a torsion balance in vacuum and the resulting rotation was used to determine the force and thus the momentum change.

The Jones-Richardson experiment, performed first in 1954 measured the recoil force on a mirror in a dielectric liquid[56] and found a result that supported the Minkowski momentum. Significant improvements were made by Jones and Leslie in 1977 [57], including the use of a high-reflectance dielectric mirror to reduce convection currents. The authors of the 1977 paper claim that the radiation pressure force increases linearly with the phase refractive index, to an accuracy of $0.05 \%$.

An alternate measurement was performed by Ashkin and Dziedzic in 1973 which measured the force on a liquid surface due to a laser beam exiting the medium [58]. An outward curvature of the surface was observed, consistent with the Minkowski form, using a pulsed laser with a $4 \mathrm{~kW}$ peak power.

An experiment performed in 2002 by Graebner et al. [41] examined the motion of a MEMS mirror in response to the force exerted by an incident laser beam. An optical power of $3 \mathrm{~mW}$ was used to generate deflections on the

\footnotetext{
${ }^{4}$ In fact, simple observation shows that the net force on the vane is directed towards the dark, or less reflective side. This side absorbs more energy, but as can be readily calculated, the radiation pressure force is greatest on the reflective surface. Quite simply, the Crooke's radiometer turns in the wrong direction for the motion to be due to radiation pressure.
} 
$\mathrm{nm}$ scale (measured using an interferometer), giving agreement within $30 \%$ of the theory. Despite a mirror reflectivity of only $95 \%$, the authors made no attempt to characterize the thermal effects on the system, relying on the agreement with theory as proof of the effect.

In 2008, She et al. observed the recoil force due to light leaving the endface of a silica nanofiber 24] and reported a result which favors the Abraham form. The fiber diameter of only $450 \mathrm{~nm}$ leads to a very soft structure and light coupled into it leads to an observable deflection. The authors' conclusions are, however, disputed [25, 26].

Lui et al. have demonstrated a nanoscale plasmonic motor which harnesses the angular momentum of light to rotate a silica disk [59]. The authors report a torque of $140 \mathrm{pN}$ from an linearly polarized beam with an intensity of $1 \mathrm{~mW} / \mu^{2}$. Further, by changing the wavelength of the optical drive beam different resonances could be excited in the structure allowing the direction of the torque, and thus the rotation, to be reversed. This effect opens another avenue for the optical actuation of microstructures.

The primary challenge in making measurements of optical momentum is the extremely small magnitude of the resulting force. This means that not only must the experiment be very sensitive, but it must also be isolated against outside perturbations or spurious responses. A significant concern is avoiding spurious responses due to thermal effects, which may be significant. The mechanical power transfered to a system is

$$
P_{\text {mech }}=\frac{d E_{\text {mech }}}{d t}=\frac{d}{d t} \frac{p_{\text {mech }}^{2}}{2 m}=\frac{p_{\text {mech }}}{m} \frac{d p_{\text {mech }}}{d t}
$$

Using the optical force $F=P_{\text {opt }} / c$ this becomes

$$
P_{\text {mech }}=\frac{p_{m e c h} P_{o p t}}{m c}
$$

The thermal power transfered to the system is simply

$$
P_{\text {therm }}=A P_{\text {opt }}
$$

where $A$ is the total optical absorption in the beam path. For a mirror $A$ equals one minus the reflection coefficient, or about $1 \%$ for typical metal mirrors.

Due to this issue, experiments attempting to measure optical forces must take care to demonstrate that optical momentum is the cause of the observed force, and not thermal effects. Techniques have included decreasing absorption using dielectric mirrors [57, high vacuum to prevent convection currents [3] and thermal time constants longer than the optical response frequency. 
McMaster University - Eng. Physics

\subsubsection{Resolution}

The Abraham-Minkowski controversy has been a subject of debate for the last century due to the simple yet fundamental nature of the question. Experimental resolution has been difficult, owing both to the small forces involved and the apparently contradictory results. Recently a number of publications have offered ${ }^{5}$ a resolution to this controversy which accepts both forms, provided a correct analysis is followed. [21, 22, 23] A simple summary might suggest that the Abraham form represents the Kinetic momentum of the photon while the Minkowski form represents the Canonical momentum.

The arguments presented above for the Abraham momentum are based on the conservation of kinetic momentum and the motion of mass-energy. These mechanical arguments give the correct result when considering the force due to light entering or leaving the medium and in other cases where the kinetic momentum is involved.

The canonical or Minkowski momentum arises in cases where the momentum is quantum in nature and satisfies the canonical commutation relation $[x, p]=i \hbar$. This implies that the momentum is given by the momentum operator $p=-i \hbar \nabla$. Alternately, this is the correct approach where the wavelength is the fundamental property and momentum is given by $p=h / \lambda$ and the wavelength in the medium must be used.

\subsection{Optical Forces}

\subsubsection{Derivation of radiation pressure}

From (2.4), photons carry a momentum with magnitude

$$
p=\frac{E}{c}=\frac{h f}{c}
$$

proportional to their energy. Should a photon with this momentum encounter a reflecting surface, conservation of momentum gives ${ }^{6}$

$$
\begin{gathered}
p_{\gamma i}+p_{R i}=p_{\gamma f}+p_{R f} \\
\frac{h f}{c}+0=-\frac{h f}{c}+\Delta p_{R}
\end{gathered}
$$

where $p_{\gamma}$ and $p_{R}$ are the momenta of the photon and the reflector before and after the interaction. Thus the momentum change experienced by the reflector

\footnotetext{
${ }^{5}$ Or boldly claimed as in 22,23

${ }^{6}$ Assuming a perpendicular angle of incidence
} 
i氵7

$$
\Delta p_{R}=2 \frac{h f}{c}=2 p_{\gamma}
$$

Force on an object is equal to its change in momentum with time

$$
\vec{F}=\frac{d \vec{p}}{d t}
$$

from Newton's second law. In the case of a single photon the interaction time $d t$ is not clear, but in the more practical (and therefore more interesting) case of many photons arriving at a constant rate this becomes

$$
\begin{aligned}
F & =2 \frac{d}{d t} \frac{E}{c} \\
& =2 \frac{P}{c}
\end{aligned}
$$

which is a force proportional to the power incident on the reflecting surface. It is traditional to consider the pressure on a surface

$$
\mathcal{P}=2 \frac{P}{c A}=2 \frac{S}{c}
$$

where $A$ is the surface area receiving the force and $S$ is the time-averaged Poynting vector.

If, instead of being reflected the photon is simply deflected by an angle $\theta$ due to its interaction with some structure, momentum conservation gives

$$
\begin{gathered}
\vec{p}_{\gamma f}=\left(p_{0} \cos \theta, p_{0} \sin \theta\right) \\
\Delta \vec{p}_{i}=\left(p_{0}(1-\cos \theta),-p_{0} \sin \theta\right)
\end{gathered}
$$

for the interaction shown in Figure 2.3. It is interesting to note that this interaction is essentially a 'black box' and nothing need be assumed about the nature of this interaction. If there are many photons passing into the interaction at a constant rate then the above approach may be repeated, giving a force

$$
\vec{F}=\frac{P}{c}(1-\cos \theta,-\sin \theta)
$$

on the structure with which the photons are interacting. The scalar magnitude of this force is

$$
\|F\|=2 \frac{P}{c} \sin \frac{\theta}{2}
$$

which reduces to the form in Equation 2.35) for $\theta=\pi / 2$.

\footnotetext{
${ }^{7} \mathrm{An}$ absorbing surface experiences half this momentum change as the final momentum of the photon in that case is zero.
} 
Masters Thesis - Christopher Pope McMaster University - Eng. Physics

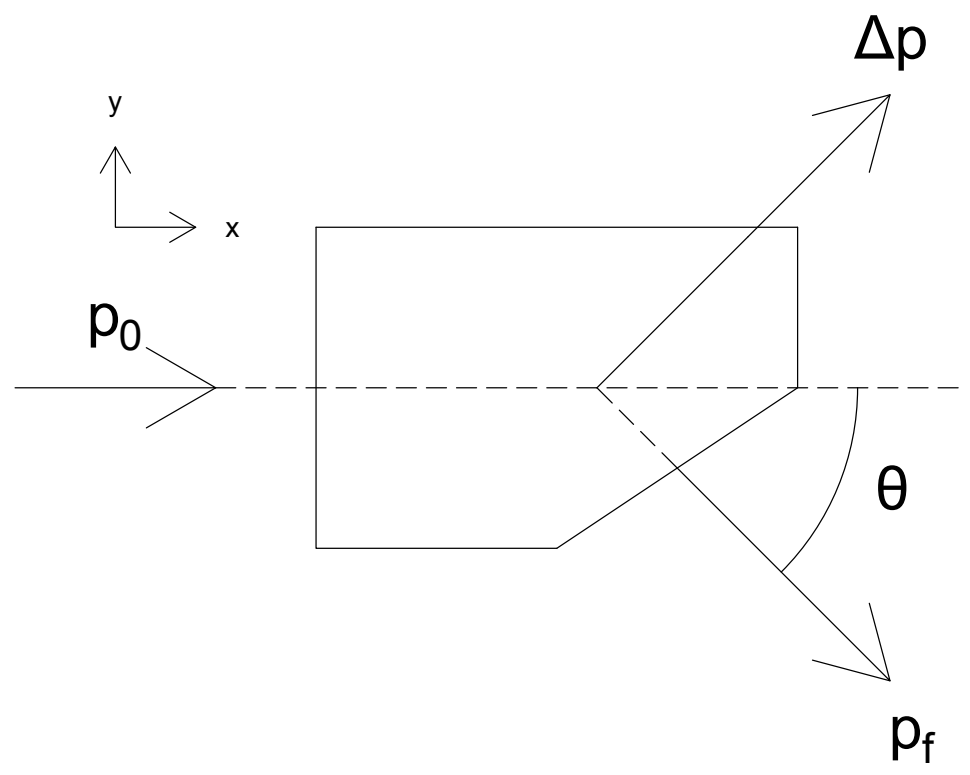

Figure 2.3: Diagram illustrating the scattering of a photon from an arbitrary interaction. The change in momentum of the interacting object is $\Delta \vec{p}=-\left(\vec{p}_{f}-\right.$ $\left.\vec{p}_{0}\right)$.

\subsubsection{Forces on waveguides}

The optical force acting on a waveguide may be understood by considering the case of a particle constrained to move along a curved path. If the particle has some initial momentum $\vec{p}_{0}$ then by Newton's second law it must experience a force

$$
\vec{F}=\frac{d \vec{p}}{d t}
$$

to keep it on that path. If the movement along the path is frictionless it is only the direction of the momentum vector that changes, not the magnitude. At any point along the path the particle is traveling in a direction tangent to the path. If the path is described by a function $\vec{u}(x)$ then the momentum vector can be written

$$
\vec{p}=p_{0} \frac{d \vec{u}}{d x}
$$

and the force is thus

$$
\begin{aligned}
\vec{F} & =\frac{d}{d t}\left(p_{0} \frac{d u}{d x}\right) \\
& =p_{0} \frac{d}{d x}\left(\frac{d u}{d x}\right) \frac{d x}{d t} \\
& =p_{0} c \frac{d^{2} \vec{u}}{d x^{2}}
\end{aligned}
$$


where $c$ is the speed at which the particle travels along the path.

The above derivation makes no assumptions about the nature of the particle and Newton's second law is used in the relativistic form. Therefore, the conclusion in Equation (2.45) is valid for the case of a photon confined in a waveguide. By Newton's third law the waveguide experiences an equal and opposite force, which acts to oppose the curvature. This force is proportional to the curvature of the waveguide and represents an optical stiffness due to the optical energy confined in the waveguide.

The simplest case is a short waveguide section with radius of curvature $R$. The curvature of the waveguide is then $1 / R$ and the optical force has magnitude

$$
F=\frac{p_{0} c}{R}
$$

directed radially inwards. Substituting the free-space photon momentum gives a force

$$
F=h f \frac{1}{R}
$$

per photon in the waveguide. The total force exerted by multiple photons is thus found simply by integrating over the photon energy $h f$ of all the photons in the waveguide.

From an experimental perspective it is more convenient to consider the optical power $P$ in the waveguide. The linear energy density in the waveguide is the power divided by the propagation speed. Thus, the linear force density in the curved waveguide is

$$
\frac{F}{L}=\frac{P}{c R}
$$

assuming the photons are propagating in free space. The Minkowski and Abraham forms are

$$
\begin{aligned}
F_{\text {Min }} & =\frac{n P}{c R} \\
F_{A b r} & =\frac{P}{n c R}
\end{aligned}
$$

respectively, as might be expected.

While the analytical expression in (2.48) for the optical force on a structure with a constant curvature can be derived in a straightforward manner, the situation for a beam is more complicated. The optical force is proportional to the curvature, but the curvature depends on the displacement of the beam and is, in fact, not constant along the length of the beam. Thus, the EulerBernoulli beam equation becomes

$$
\rho A \frac{d^{2} u}{d t^{2}}+\frac{d^{2}}{d t^{2}}\left(E I \frac{d^{2} u}{d x^{2}}\right)=f(x, t)-\frac{P}{c} \frac{d^{2} u}{d x^{2}}
$$


where the optical force effectively adds to the mechanical stiffness of the beam.

If the beam is a cantilever it can be seen readily from the mode shap $\AA^{8}$ that the net force applied by light passing through the structure will apply a force which opposes the curvature of the beam. This follows the same form as outlined in Figure 2.3. The magnitude of this force is estimated below.

The situation for a bridge is more complicated than a cantilever. The curvature of a bridge changes sign at a distance of $L / 4$ from each end. The integral of this curvature over the bridge length is zero and thus from 2.45) the net optical force on a bridge is zero. This can also be seen by simply considering either a photon or the optical mode at each end of the bridge. As the momentum is identical after passing through the bridge (assuming lossless propagation) there is no net change in momentum and thus no net force.

Despite the lack of a net force there is still an optical force acting on each point of the bridge and this force may produce a measurable effect. The bridge stiffness in response to a point load varies along the bridge, being roughly inversely proportional to the mode shape. Thus the center half of the bridge will have a greater response to the optical force than the stiffer ends, and this will lead to an effect on the mode 9

\subsubsection{Optical stiffness}

Any constant $k$ that fits the form of Hooke's law

$$
F=-k x
$$

may be called a stiffness ${ }^{10}$ In the case of a cantilever of length $L$ clamped at one end and deflected by an angle $\theta$ at the free end, the linear deflection can be approximated as $x=L \theta$ for small angles. The force caused by this deflection is given by Equation (2.40) as

$$
F=2 \frac{P}{c} \sin \frac{\theta}{2}
$$

which reduces to

$$
F=\frac{P}{c} \theta
$$

in the case of small angular deflections. The optical "stiffness" of this structure under a linear deflection $x$ is then

$$
k=\frac{F}{x}=\frac{P}{c L}
$$

\footnotetext{
${ }^{8}$ The mode shapes for simple beams will be discussed in Section 2.3.3.

${ }^{9}$ This effect on bridge deformation is demonstrated by accident in Section 3.4 .2 by means of thermally induced strains.

${ }^{10}$ It may be noted that the stiffness of a structure depends on the distribution of the applied force, and on the definition of the resulting deflection.
} 


\section{$2.3 \quad$ Elasticity}

The majority of MEMS devices are designed around flexural motion and thus an understanding of elasticity is the basis for the mechanical analysis of many microsystems. Elastic deformations have small energy losses compared to frictional losses in translational motion.

\subsubsection{Stress and strain}

A beam of length $L$ which is stretched in the x-direction to a length $L+\Delta L$ is said to experience a strain ${ }^{11}$

$$
\epsilon_{x x}=\frac{\Delta L}{L}
$$

which is the fractional extension of the beam. If the beam has a cross-sectional area $A$ perpendicular to the extension and is acted on by a force $F$, then the stress in the direction of extension is

$$
\sigma_{x x}=\frac{F_{x}}{A_{x}}
$$

Identical expressions can be derived in the $\mathrm{y}$ and $\mathrm{z}$-directions for a threedimensional object. In the general case in three dimensions, both stress and strain are symmetric tensors. Subscripts here indicate direction components of these tensors.

For most materials undergoing small extensions the stress is proportional to the strain

$$
\begin{aligned}
\sigma_{x x} & =E \epsilon_{x x} \\
\frac{F_{x}}{A_{x}} & =E \frac{\Delta L}{L}
\end{aligned}
$$

where the proportionality constant $E$ is the Young's modulus or tensile modulus. This expression is another form of Hooke's law. A solid object typically does not deform in only one direction at a time. An extension in the $\mathrm{x}$ direction is accompanied by contractions in the y and z-directions. For an isotropic material the resulting stresses are given by

$$
\sigma_{y y}=\sigma_{z z}=-\nu \sigma_{x x}
$$

where the proportionality constant $\nu$ is known as Poisson's ratio. Shear stresses $\sigma_{i j}$ also exist, which act in the plane of the cross-section $i$ in the $j$ direction.

\footnotetext{
${ }^{11}$ See Landau [60] or Kaajakari [27, ch. 4] for a more extensive overview
} 
In general the elastic constants of a material need not be the same in all directions and the material may be anisotropic. For anisotropic materials, Hooke's law becomes

$$
\sigma_{i j}=E_{i j k \ell} \epsilon_{k \ell}
$$

where $E_{i j k \ell}$ is the stiffness tensor. Symmetries reduce the number of possible unique coefficients for this tensor from 81 to 21 .

Silicon is a material in which the elastic behavior cannot be completely expressed by a single parameter. The elastic constants for silicon are [61]

$$
\begin{gathered}
E_{11}=E_{x x x x}=165.6 \mathrm{GPa} \\
E_{12}=E_{x x y y}=63.9 \mathrm{GPa} \\
E_{44}=E_{y z y z}=79.5 \mathrm{GPa}
\end{gathered}
$$

with only three independent components from cubic symmetry. These elastic constants are for the crystal with axes along the $\langle 100\rangle$ and related directions. Uniaxial tension or compression is governed by the value of $E$ along the direction of tension, which can be calculated from the equation

$$
\frac{1}{E_{h k \ell}}=s_{11}-2\left[\left(s_{11}-s_{12}\right)-\frac{1}{2} s_{44}\right]\left(m^{2} n^{2}+n^{2} p^{2}+m^{2} p^{2}\right)
$$

where $m, n$, and $p$ are the direction cosines giving the angles between the $\langle h k \ell\rangle$ direction and the $\langle 100\rangle$ axes. The coefficients are from the inverse elasticity matrix and are equal to

$$
s_{11}=7.68 \times 10^{-12} \mathrm{~Pa}^{-1} \quad s_{12}=-2.41 \times 10^{-12} \mathrm{~Pa}^{-1} \quad s_{44}=12.6 \times 10^{-12} \mathrm{~Pa}^{-1}
$$

in silicon. Values of this equation computed along the principle crystal directions of interest are

$$
E_{100}=130 \mathrm{GPa}, \quad E_{110}=169 \mathrm{GPa}, \quad E_{111}=188 \mathrm{GPa}
$$

Another direction of interest is the $\langle 211\rangle$ direction, which is perpendicular to both the $\langle 110\rangle$ and $\langle 111\rangle$ directions. The Young's modulus in this direction is

$$
E_{211}=173 \mathrm{GPa}
$$

\subsubsection{Simple beams}

The deformation of a simple beam undergoing small displacements is described by the Euler-Bernoulli beam theory. The derivation begins by considering a 
small beam element of length $d x$, as shown in Figure $2.4^{12}$ The beam is deflected from its rest position by a distance $u(x)$. In dynamic equilibrium the inertial and shear forces balance

$$
0=V-\left(V+\frac{\partial V}{\partial x} d x\right)-\rho A d x \frac{\partial^{2} u}{\partial t^{2}}
$$

as do the torques

$$
V d x=\frac{\partial M}{\partial x} d x
$$

Substituting the value for $V$ from (2.70) into (2.69) gives

$$
\frac{\partial^{2} M}{\partial x^{2}} d x=-\rho A d x \frac{\partial^{2} u}{\partial t^{2}}
$$

Knowing that the bending moment $M$ is proportional to the beam curvature

$$
M=E I \frac{\partial^{2} u}{\partial x^{2}}
$$

gives the Euler-Bernoulli beam equation

$$
\rho A \frac{d^{2} u}{d t^{2}}+\frac{d^{2}}{d t^{2}}\left(E I \frac{d^{2} u}{d x^{2}}\right)=f(x, t)
$$

Here $\rho$ is the beam's mass density, $E$ is its Young's modulus, $A$ is the crosssectional area and $I$ is the second moment of inertia, given by

$$
\begin{aligned}
& I_{r}=\frac{w t^{3}}{12} \\
& I_{c}=\frac{\pi r^{4}}{4}
\end{aligned}
$$

for rectangular and circular beams respectively. The thickness $t$ of a rectangular beam is measured in the direction of displacement while the width $w$ is measured perpendicular to that displacement.

\subsubsection{Solutions: bridges and cantilevers}

The natural resonant shapes, or mode shapes of a beam are found from the solutions of Equation (2.73) with zero applied force. The resonant frequencies are the corresponding eigenfrequencies. When $E$ and $I$ are constants the equation is separable, and we seek a solution

$$
u(x, t)=u(x) T(t)=C e^{i x B} \sin \omega t
$$

\footnotetext{
${ }^{12}$ Following the example of Weaver 62].
} 
Masters Thesis - Christopher Pope $\quad$ McMaster University - Eng. Physics

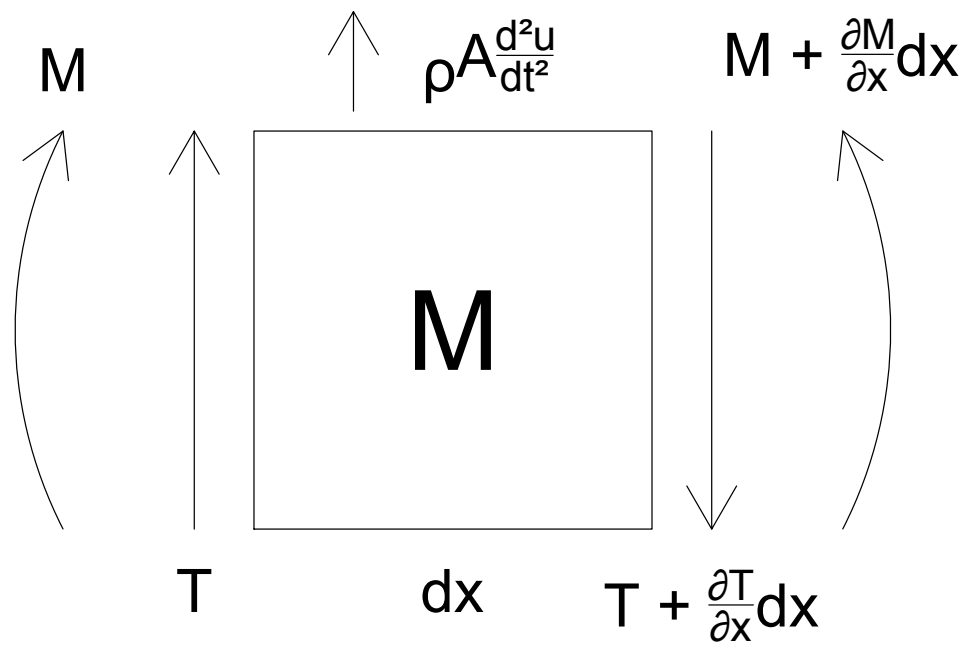

Figure 2.4: Diagram the bending of a differential beam element used in EulerBernoulli beam theory.

where $C$ is a constant. Computing the solutions is straightforward and only the results will be presented ${ }^{13}$ The solutions support a number of modes determined by the constants $\beta_{n}$, which are roots of a characteristic equation that depends on the boundary conditions.

The spatial component is determined by a fourth-order ordinary differential equation which has four constants and requires four boundary conditions. Two cases are of primary interest in MEMS devices, the bridge (fixed-fixed beam) and the cantilever (fixed-free beam). These are discussed in detail below, however general expressions for the resonant frequency and stiffness can be determined. The resonant frequency of a beam is given by

$$
\omega=\beta_{n}^{2} \sqrt{\frac{E I}{\rho A L^{4}}}
$$

and the corresponding stiffness is

$$
k_{n}=\omega_{n}^{2} m=\omega_{n}^{2} \rho A L k_{n}=\beta_{n}^{4} \frac{E I}{L^{3}}
$$

In the common case that the beam has a rectangular cross-section with a thickness $t$ in the direction of bending and a width $\mathrm{w}$, the above expressions

\footnotetext{
${ }^{13}$ Further analysis can be found in Weaver, Timoshenko and Young 62.
} 
reduce to

$$
\begin{gathered}
\omega=\beta_{n}^{2} \frac{t}{L^{2}} \sqrt{\frac{E}{12 \rho}} \\
k_{n}=\beta_{n}^{4} \frac{E w t^{3}}{12 L^{3}}
\end{gathered}
$$

which are useful forms for most MEMS structures. It should be noted that the relationship between force and displacement for a beam will give a slightly different stiffness depending on how the force is distributed, although the form will be similar.

The fixed-fixed boundary condition describes structures where both ends are firmly affixed to some support structure. In this case both the mode shape and the slope (first derivative) are constrained to be zero at the ends

$$
u(0)=u(L)=u^{\prime}(0)=u^{\prime}(L)=0
$$

The mode shape of a fixed-fixed beam is given by

$$
\begin{gathered}
u_{n}(x)=\sinh \beta_{n} x / L-\sin \beta_{n} x / L+a_{n}\left(\cosh \beta_{n} x / L-\cos \beta_{n} x / L\right) \\
a_{n}=\frac{\sinh \beta_{n}-\sin \beta_{n}}{\cos \beta_{n}-\cosh \beta_{n}}
\end{gathered}
$$

where $\beta_{1}=4.73004$. This mode shape is illustrated in Figure 2.5, along with the first and second derivatives. The constants $\beta_{n}$ are the roots of the equation

$$
\cos \beta_{n} \cosh \beta_{n}=1
$$

The fixed-free boundary condition describes cantilever structures where one end may move freely without constraint. This lack of constraint implies that the bending moment and shear force are zero, corresponding to the second and third derivatives

$$
u(0)=u^{\prime}(0)=u^{\prime \prime}(L)=u^{\prime \prime \prime}(L)=0
$$

The mode shape of a fixed-free beam is given by

$$
\begin{gathered}
u_{n}(x)=\sin \beta_{n} x / L-\sinh \beta_{n} x / L-a_{n}\left(\cos \beta_{n} x / L-\cosh \beta_{n} x / L\right) \\
a_{n}=\frac{\sin \beta_{n}+\sinh \beta_{n}}{\cosh \beta_{n}+\cos \beta_{n}}
\end{gathered}
$$

where $\beta_{1}=1.87510$. This mode shape is illustrated in Figure 2.6, along with the first and second derivatives. The constants $\beta_{n}$ are the roots of the equation

$$
\cos \beta_{n} \cosh \beta_{n}=-1
$$


Masters Thesis - Christopher Pope McMaster University - Eng. Physics

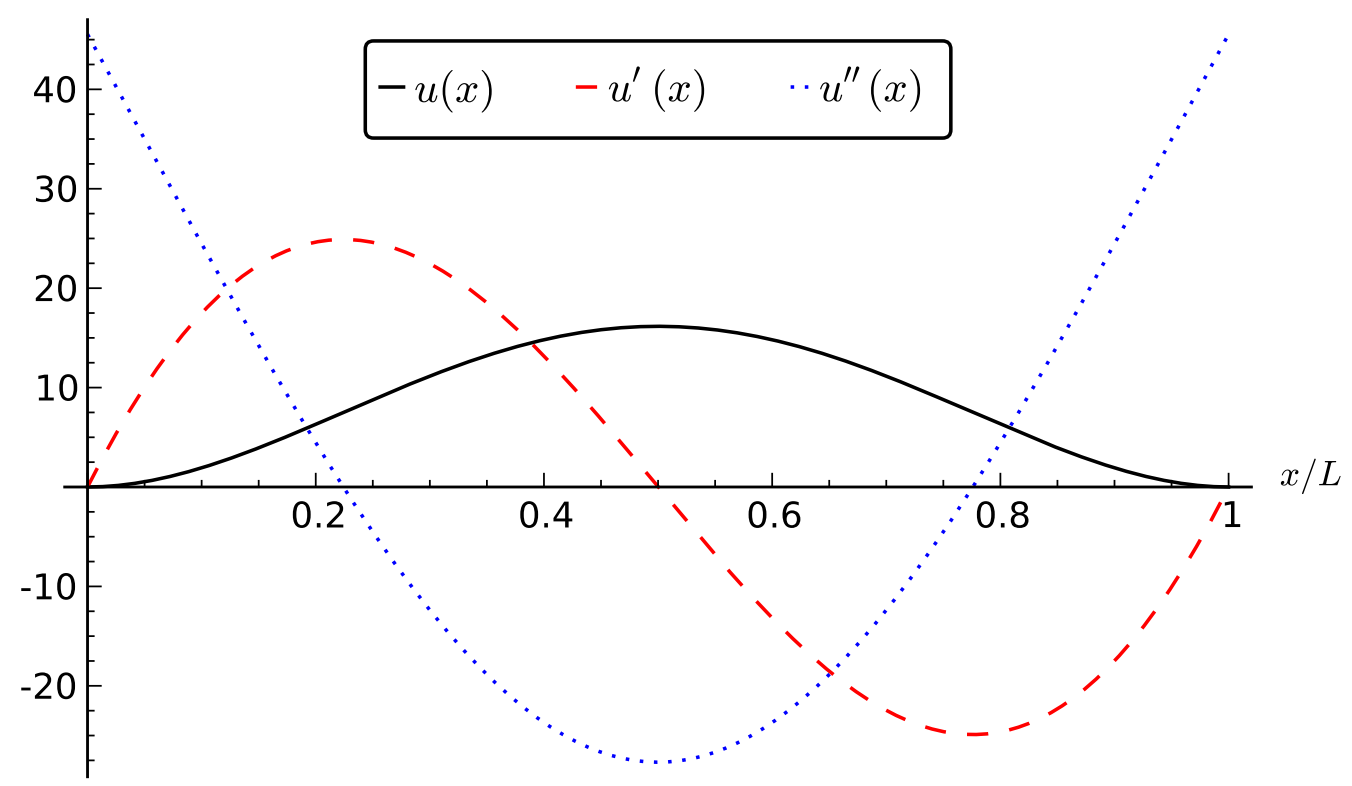

Figure 2.5: Mode shape and its first two derivatives for a fixed-fixed beam (or bridge). Vertical axis is in arbitrary units

\subsection{Waveguides}

A wide variety of structures have been developed to guide the propagation of electromagnetic waves. Similar techniques can be used to analyze fiber optic cables for telecommunications, microwave or radio-frequency waveguides for wireless communications and coaxial cables used for television and numerous other applications.

Waveguides are understood by analyzing the effects of the boundary conditions they impose on the electric and magnetic fields ${ }^{14}$ These boundary conditions allow for the propagation of waves in a number of modes, which describe the electromagnetic field intensity in the waveguide. Maxwell's equations are scale-invariant and thus the analyses presented below are independent of frequency. Practical considerations such as absorption and manufacturing typically dictate the choice of materials.

Many waveguides allow modes to propagate above a cutoff frequency determined by the shape and dimensions of the structure. For a metal-walled waveguide having a rectangular cross-section with dimensions $(a, b)$ the cutoff frequency for TE modes is given by

$$
f_{c(m, n)}=\frac{1}{2 c} \sqrt{\left(\frac{m}{a}\right)^{2}+\left(\frac{n}{b}\right)^{2}}
$$

${ }^{14} \mathrm{~A}$ brief overview of waveguide theory is provided here. Detailed analyses can be found in Ida 63] for metallic waveguides or Reed and Knights 64 for dielectric waveguides. 
Masters Thesis - Christopher Pope McMaster University - Eng. Physics

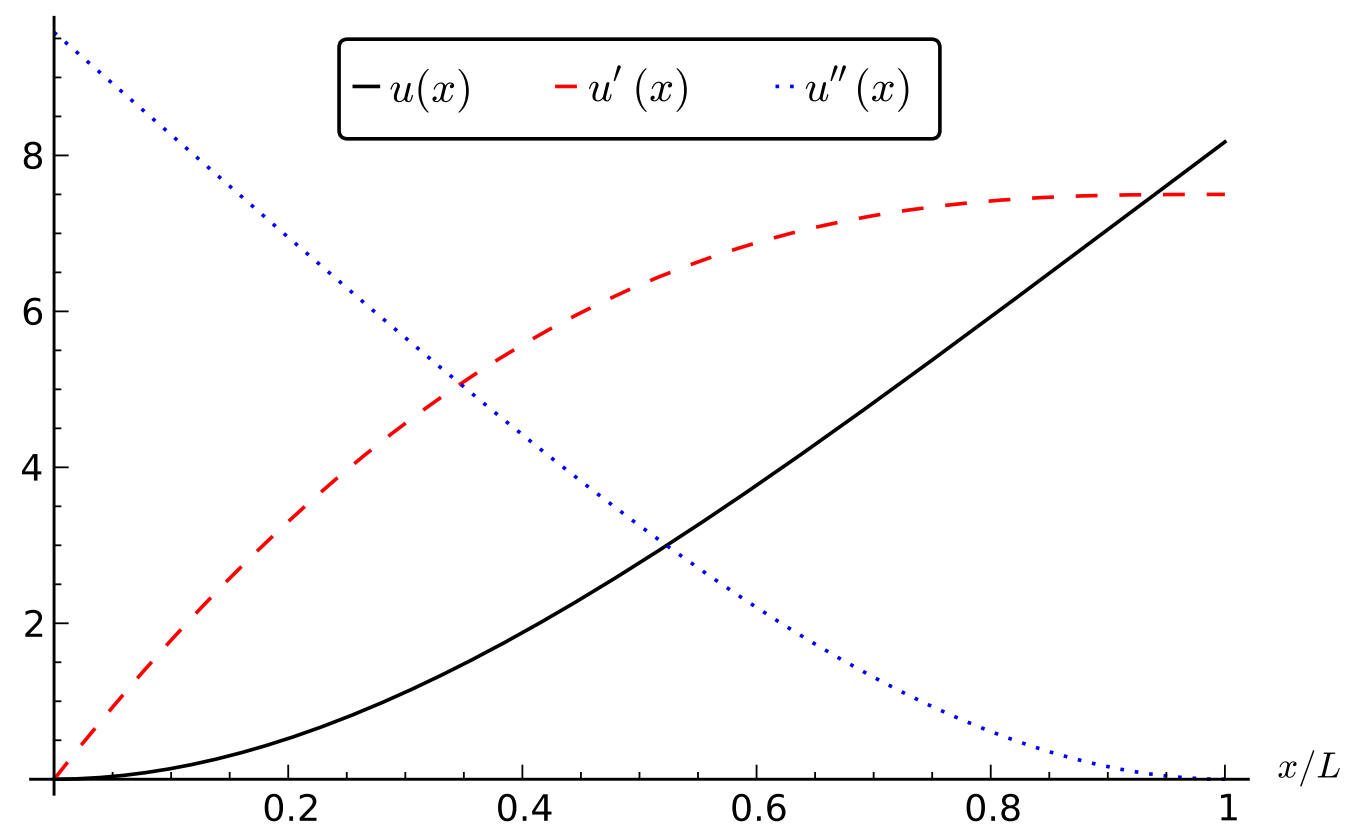

Figure 2.6: Mode shape and its first two derivatives for a fixed-free beam (or cantilever). Vertical axis is in arbitrary units.

where $m, n \in\{0,1,2 \ldots\}$ are called the mode numbers. 63] It is often desirable to have only a single mode propagating in a waveguide as modes differ in propagation speeds. Thus, multi-mode propagation can cause dispersion, with the energy in each mode arriving at an endpoint at slightly different times. This leads to pulses broadening and waveforms distorting, which is detrimental to communications systems.

\subsubsection{Dielectric waveguides}

Electromagnetic waves, typically light, can also be confined inside a dielectric by means of total internal reflection. When a light beam travels from one medium into another it experiences refraction, appearing to bend at the interface. Refraction is described by Snell's law

$$
n_{i} \sin \theta_{i}=n_{t} \sin \theta_{t}
$$

where $n_{i}, n_{t}$ are the indices of refraction on the incident and transmitted sides of the interfaces, and $\theta_{i}, \theta_{t}$ are the corresponding angles the light beam forms with the surface normal. 65] Total internal reflection occurs when the transmitted angle reaches $90^{\circ}$ and the light no longer propagates into the second 
medium. This occurs at an angle of incidence

$$
\theta_{i}=\theta_{c}=\sin ^{-1} \frac{n_{t}}{n_{i}}
$$

called the critical angle. Clearly light is reflected from a larger range of angles as the refractive index difference increases, with $n_{i}>n_{t}$.

If the incident medium is a slab with two parallel surfaces then the light beam can be confined over long distances. If a light beam is totally reflected then it was incident and is reflected at an angle $\theta>\theta_{c}$. However, it will also be incident at the same angle $\theta$ on the other parallel surface and be reflected in the same way. Two-dimensional confinement can be arranged by adding another pair of parallel surfaces perpendicular to the first. A similar line of reasoning can be used in circular geometries such as optical fibers.

\subsubsection{SOI waveguides}

Recent advances in silicon-based manufacturing techniques, combined with its low cost compared to III-V semiconductors and the potential to integrate electronic circuitry has created an interest in silicon-based optical structures, including waveguides. 64] Silicon is transparent in the infrared, for wavelengths longer than $1.1 \mu \mathrm{m}$ and has a high dielectric constant, roughly 3.5 around the commonly used telecom wavelength of $1.5 \mu \mathrm{m}$.

A popular method for manufacturing waveguides out of silicon is the siliconon-insulator (SOI) rib waveguide, illustrated in Figure 2.7. Single-mode waveguides in this geometry can be designed with heights of several microns, significantly larger than the roughly $200 \mathrm{~nm}$ maximum for a rectangular waveguide. The theory behind this geometry was first demonstrated by Soref et al. [66].

For a rib waveguide with a rib height of $2 \lambda b$, a rib width of $2 \lambda a$ and a slab height of $2 \lambda r b$ the single-mode condition is

$$
\frac{a}{b} \leq C+\frac{r}{\sqrt{1-r^{2}}}
$$

where $C$ is a fitting constant. The original value was 0.3 from Soref et al., but a stronger condition of $C=0$ was imposed by Pogossian et al [67], who also suggests using $C=-0.05$ for design purposes. This is an approximation valid for the case of large rib height $(b>1)$ and large slab heights $(r>0.5)$.

\subsection{Electromechanical systems}

\subsubsection{Harmonic motion}

Physical phenomena as diverse as guitar strings and orbiting planets may be understood in terms of a periodic motion. This commonality makes the study 
Masters Thesis - Christopher Pope $\quad$ McMaster University - Eng. Physics

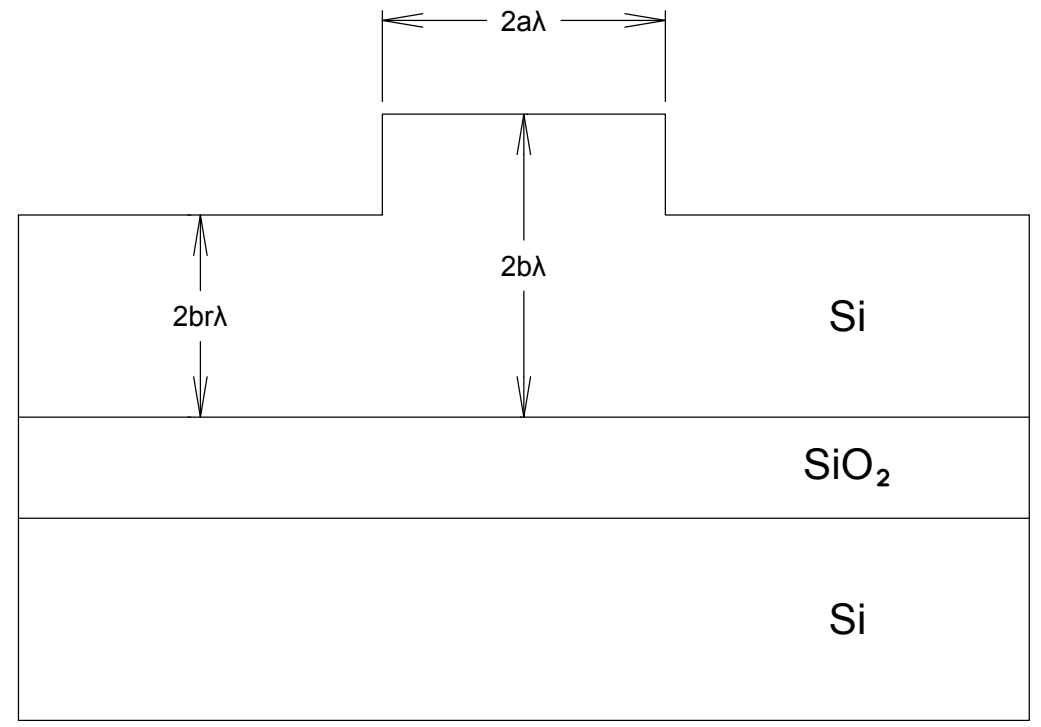

Figure 2.7: Drawing of an SOI rib waveguide

of oscillations a fundamental technique for analyzing physical systems. The general approach usually taken is to consider the simple harmonic oscillator, 68. a one-dimensional system where a point mass $m$ moves in a potential

$$
U=\frac{1}{2} k x^{2}
$$

with a minimum at $x=0,{ }^{15}$ The force on an object due to this potential is

$$
F=-\frac{d U}{d x}=-k x
$$

which is the familiar form of Hooke's law. When combined with Newton's second law this yields the equation of motion

$$
\frac{d^{2} x}{d t^{2}}+\frac{k}{m} x=0
$$

The solution is well-known to be

$$
x(t)=A \sin \omega_{0} t
$$

with the constant

$$
\omega_{0}=\sqrt{\frac{k}{m}} \quad T=\frac{2 \pi}{\omega_{0}}=\frac{1}{f}
$$

\footnotetext{
${ }^{15}$ While higher-order terms may appear in real potentials, they are neglected in the simple case. Both the gravitational and electrostatic potentials are of this form.
} 
Masters Thesis - Christopher Pope McMaster University - Eng. Physics

being the angular resonant frequency. The motion is periodic with a period $T$.

In general a system undergoing harmonic motion is often subject to some dissipative force, which is typically a function of the system's velocity. For small velocities this is given by

$$
F=-b \frac{d x}{d t}
$$

where $b$ is a constant related to the loss mechanism ${ }^{16}$ The resulting equation of motion is

$$
m \frac{d^{2} x}{d t^{2}}+b \frac{d x}{d t}+k x=0
$$

Substituting the resonant frequency from (2.97) and a damping factor

$$
\gamma=\frac{b}{2 m}
$$

results in

$$
\frac{d^{2} x}{d t^{2}}+2 \gamma \frac{d x}{d t}+\omega_{0}^{2} x=0
$$

The general solutions are of the form

$$
x(t)=A e^{a t}, \quad a=\gamma \pm \sqrt{\gamma^{2}-\omega_{0}^{2}}
$$

where the constant $a$ determines the behavior of the system. In the case $\gamma^{2}<\omega_{0}^{2}$ the constant is complex and the solution can be expressed as

$$
x(t)=A e^{-\gamma t} \sin \left(\omega_{d} t+\phi_{0}\right)
$$

where the damped resonant frequency is

$$
\omega_{d}=\sqrt{\omega_{0}^{2}-\gamma^{2}}
$$

This solution behaves like free oscillation modulated by an exponential decay.

\footnotetext{
${ }^{16}$ This is the first term of a Taylor series expansion of the function $F(\dot{x})$. At higher velocities the quadratic term often dominates, but the solution for the linear term is simpler.
} 


\subsubsection{Resonance}

It is common to consider the behavior of an oscillating system that is subject to an external force

$$
F=F_{0} \sin \omega t \quad \text { or } \quad F=F_{0} e^{i \omega t}
$$

which is itself periodic in time. The former form is more typical for describing the driving force while the latter is more convenient for analysis. The equation of motion is then

$$
m \frac{d^{2} x}{d t^{2}}+b \frac{d x}{d t}+k x=F_{0} e^{i \omega t}
$$

The steady-state solution is a response

$$
x(t)=A e^{i(\omega t-\phi)}
$$

at the driving frequency $\omega$ with a phase shift $\phi$ relative to the driving force. The amplitude $A$ and phase shift are given by

$$
\begin{gathered}
A=\frac{F_{0} / m}{\sqrt{\left(\omega_{0}^{2}-\omega^{2}\right)^{2}+4 \gamma^{2} \omega^{2}}} \\
\tan \phi=\frac{2 \gamma \omega}{\omega_{0}^{2}-\omega^{2}}
\end{gathered}
$$

The phase shift is zero at low frequencies and $180^{\circ}$ at high frequencies, passing through $90^{\circ}$ at the free resonance frequency $\omega_{0}$. In the undamped case the phase shift is discontinuous at $\omega=\omega_{0}$ while the amplitude is infinite. The resonance behavior in the damped case is plotted in Figure 2.8 for a normalized frequency $\omega / \omega_{0}$ with a damping coefficient $\gamma=\omega_{0} / 10$. This resonance condition is useful for improving the signal output from MEMS devices.

While it may not be obvious from Equation (2.108), the amplitude resonance of a damped harmonic oscillator is not at the free resonance frequency $\omega_{0}$ but at a resonance frequency

$$
\omega_{r}^{2}=\omega_{0}^{2}-2 \gamma^{2}
$$

which is determined by differentiating (2.108) to find the maximum.

Damping is a dissipative force which implies that energy is leaving the oscillator at some rate, balanced in the steady-state case by the energy supplied by the driving force. This energy balance is responsible for the maximum amplitude at resonance

$$
A_{\max }=\frac{F_{0}}{2 \gamma \sqrt{\omega_{0}^{2}-\gamma^{2}}}
$$


Masters Thesis - Christopher Pope McMaster University - Eng. Physics

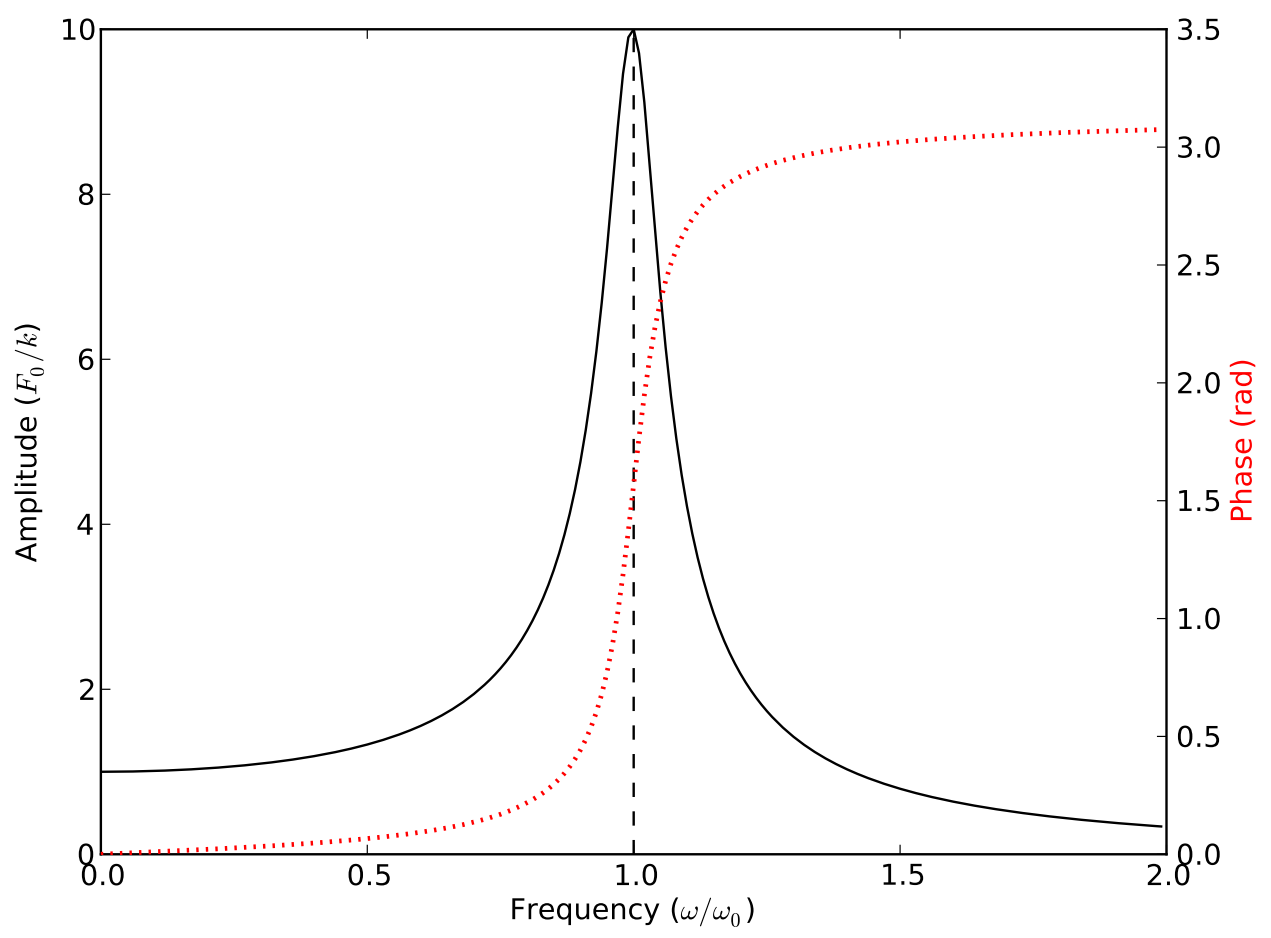

Figure 2.8: Plot of amplitude and phase as a function of a normalized frequency $\omega / \omega_{0}$ for a driven harmonic oscillator with a damping coefficient $\gamma=\omega_{0} / 10$ $(Q=5)$.

and the shape of the resonance peak. The sharpness of the peak is characterized by the quality factor $Q$ of the resonator, given by

$$
Q=2 \pi \frac{E}{\Delta E}=\frac{\omega_{d}}{2 \gamma}
$$

which may be approximated by the fractional with of the peak

$$
Q \approx \frac{\omega}{\Delta \omega}=\frac{f_{0}}{\Delta f}
$$

where the peak width $\Delta f$ is taken at the half-energy points.

\subsubsection{Capacitive detection}

Capacitive detection is commonly used to measure small deflections in MEMS devices. 27] Capacitors can be fabricated simply by a variety of methods and the resulting detectors can be quite sensitive. The capacitance between two 
parallel plates with area $A$ separated by a gap $d$ is

$$
C=\epsilon_{0} \epsilon_{r} \frac{A}{d}
$$

which is a reasonable approximation to many MEMS devices.

Measuring capacitance is performed by measuring the current through a capacitor due to an applied voltage. A capacitor caries a charge $Q=C V$ and thus the current is

$$
i=\frac{d Q}{d t}=\frac{d}{d t}(C V)
$$

for an applied voltage $V$. Expanding the derivative gives the current

$$
i=C \frac{d V}{d t}+V \frac{d C}{d t}
$$

This also illustrates the source of the two methods of measuring capacitance changes. The first term is the displacement current, used where the capacitance changes slowly relative to the applied voltage. This is the case for most capacitance meters used for measuring fixed capacitances. The second term is the motional current due to the change in capacitance. In MEMS devices this effect can be used to generate an $\mathrm{AC}$ voltage in response to mechanical motion. This effect is a natural fit with resonators, requiring only a DC bias voltage.

\subsubsection{Capacitive actuation}

Any charged object, such as the plates of a capacitor will experience an electrostatic force. The energy stored in a charged capacitor is

$$
U=\frac{Q}{2 C}=\frac{1}{2} C V^{2}
$$

where $Q$ is the charge on each plate and $V$ is the electric potential across the plates [48]. Since force is equal to the potential gradient, the force on the capacitor plates is thus

$$
F=\frac{1}{2} V^{2} \frac{d C}{d x}
$$

in the direction of increasing capacitance. For a parallel plate capacitor the change in capacitance with distance is

$$
\frac{d C}{d x}=\epsilon_{0} \epsilon_{r} \frac{A}{(d-x)^{2}}
$$

If the drive voltage is composed of an $\mathrm{AC}$ component and a $\mathrm{DC}$ component

$$
V=V_{D C}+v_{0} \cos \omega t
$$


then the force on the capacitor plate will be given by

$$
F=\frac{1}{2}\left[V_{D C}^{2}+\frac{1}{2} v_{0}^{2}+2 V_{D C} v_{0} \cos \omega t+\frac{1}{2} \cos 2 \omega t\right] \frac{d C}{d x}
$$

which gives three distinct force components. The first two terms are DC forces which set the operating point of the resonator. The third term is the response at the drive frequency. The fourth term is a response at twice the drive frequency which comes from an expansion of the $\cos ^{2}$ term $[17$

\subsubsection{Effective spring constant}

In equilibrium the electrostatic force on a beam is equal to the mechanical restoring force

$$
\begin{aligned}
F_{\text {mech }} & =F_{\text {elec }} \\
k x & =\epsilon V^{2} \frac{A}{(d-x)^{2}}
\end{aligned}
$$

using the electrostatic force for a parallel plate capacitor and the mechanical force due to Hooke's law. Solving this for the displacement $x$ due to an applied voltage is difficult, but it is quite easy to find the voltage as a function of displacement

$$
V^{2}=x(d-x)^{2} \frac{k}{\epsilon A}
$$

It is interesting to consider this equation in terms of the relative displacement $d / x$

$$
V^{2}=\frac{x}{d}\left(1-\frac{x}{d}\right)^{2} \frac{k d^{3}}{\epsilon A}
$$

which allows the behavior to be analyzed independent of the initial gap. This equation is used to generate the plot in Figure 2.9 which shows the voltage required to cause a beam displacement. The required voltage peaks at $x / d=$ $1 / 3$ and then decreases, indicating an unstable region where the stiffness of the beam is no longer able to overcome the electrostatic force. In this region the beam will tend to collapse towards the electrode in a process called pull-in or snap-down, with the potential for destructive results. The pull-in voltage is given by

$$
V_{P}=\sqrt{\frac{8}{27} \frac{k d^{3}}{\epsilon A}}
$$

\footnotetext{
${ }^{17}$ Using the identity $\cos ^{2} x=(1+\cos 2 x) / 2$
} 
Masters Thesis - Christopher Pope $\quad$ McMaster University - Eng. Physics

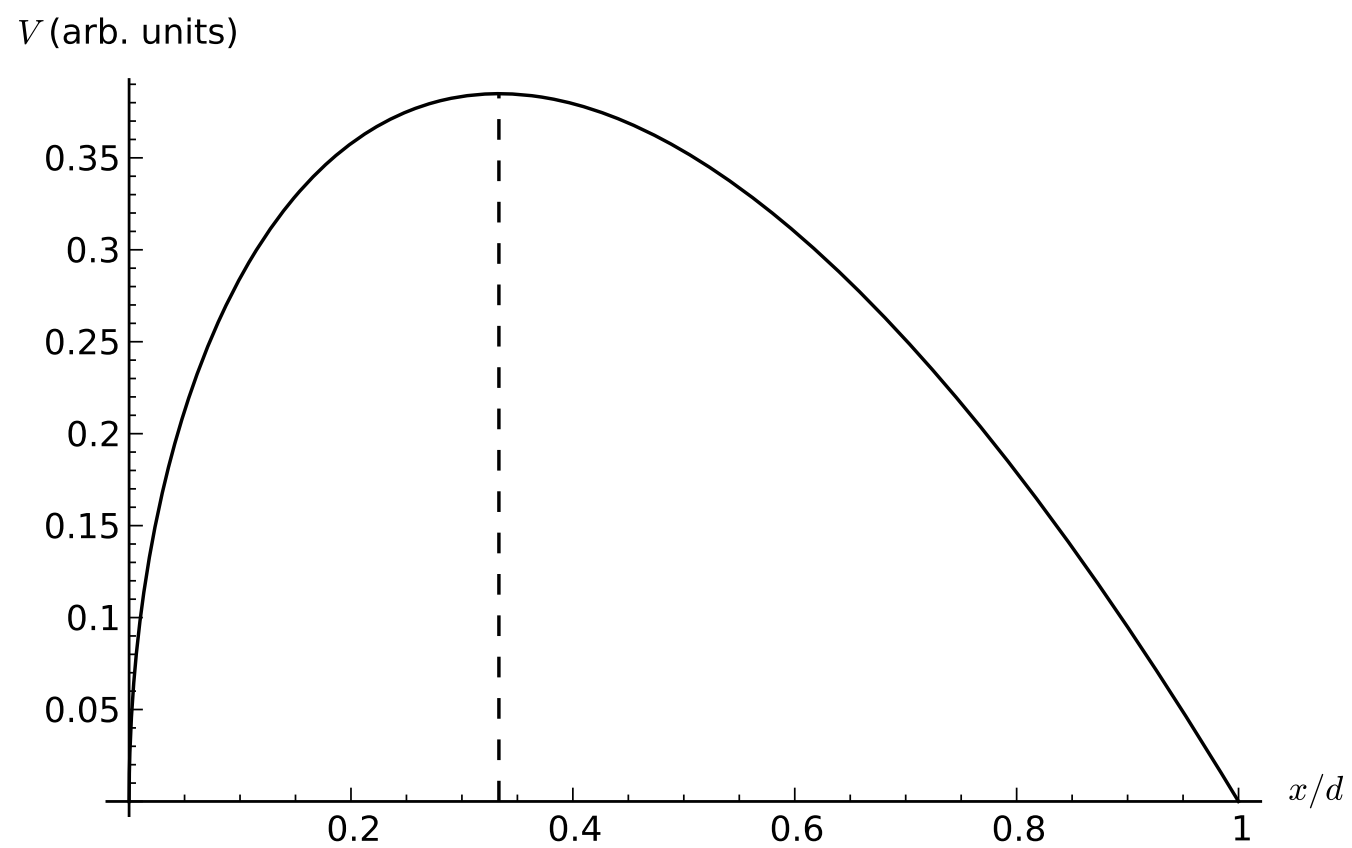

Figure 2.9: Plot of voltage vs the resulting beam deflection $x / d$ referenced to the initial gap. The decrease on the right side represents the unstable region where the beam will pull in.

Consider now the case of a beam that forms a capacitor on each side with electrodes spaced a distance $d$ from the beam. The force balance equation now reads

$$
\begin{aligned}
0 & =-k x+V^{2} \frac{\epsilon A}{d^{2}}\left(\frac{1}{(1-x / d)^{2}}-\frac{1}{(1+x / d)^{2}}\right) \\
& =-k x+V^{2} \frac{\epsilon A}{d^{2}} \frac{4 x / d}{\left[(x / d)^{2}-1\right]^{2}}
\end{aligned}
$$

with each capacitor making an equal and opposite contribution ${ }^{18}$ The equilibrium position is still $x=0$. Suppose a small force $\Delta F$ is applied to the beam. The force balance can then be written as

$$
\Delta F=k x-V^{2} \frac{\epsilon A}{d^{2}} \frac{4 x / d}{\left[(x / d)^{2}-1\right]^{2}}
$$

and we can consider an effective spring constant for this force

$$
k_{\mathrm{eff}}=\frac{\partial \Delta F}{\partial x}=k-V^{2} \frac{\epsilon A}{d^{2}} \frac{4\left(1+3 x^{2} / d^{2}\right)}{d\left[(x / d)^{2}-1\right]^{3}}
$$

${ }^{18}$ The identity $1 /(1-x)^{2}-1 /(1+x)^{2}=4 x /\left(x^{2}-1\right)^{2}$ is used 
and at $x=0$ the spring constant is

$$
\left.k_{\mathrm{eff}}\right|_{x=0}=k-V^{2} \frac{4 \epsilon A}{d^{3}}
$$

Thus, by applying a symmetric bias voltage of magnitude

$$
V=\sqrt{\frac{k d^{3}}{\epsilon A}}
$$

to both capacitor arms we can reduce the effective stiffness of the beam to zero. This technique promises to be useful for increasing the sensitivity of MEMS sensors.

\subsubsection{Electrical transduction}

This thesis proposes the use of both electrostatic actuation and capacitive sensing, using two capacitors with one side of each being formed by the moving structure. For such a device at resonance the electrostatic driving force is

$$
F=V_{D C} v_{0} \cos \omega t \frac{d C}{d x}
$$

and the current through the sensing capacitor is

$$
i=V \frac{d C}{d t}=V_{D C} \frac{d C}{d x} \frac{d x}{d t}
$$

assuming that the capacitors are identical and experience the same potential $V_{D C}$. The motion of the structure is as described above

$$
x=A e^{i(\omega t+\phi)}
$$

and the velocity is

$$
\frac{d x}{d t}=i A \omega e^{i(\omega t+\phi)}
$$

with the amplitude $A$ given by Equation (2.108). The current is then

$$
i=V_{D C}^{2}\left(\frac{d C}{d x}\right)^{2} \frac{v_{0} \omega}{m \sqrt{\left(\omega_{0}^{2}-\omega^{2}\right)^{2}+4 \gamma^{2} \omega^{2}}} e^{i(\omega t+\phi)}
$$




\section{Chapter 3}

\section{Design and Fabrication}

\subsection{Fabrication Techniques}

\subsubsection{Micromachining in Silicon}

Micromachining of silicon has been well-developed in the last decades in order to meet the needs of the semiconductor industry. 69, 29] These techniques have been leveraged to fabricate micromechanical systems for a variety of sensor applications.

The various approaches to microfabrication of microdevices are categorized as either surface micromachining or bulk micromachining. Surface micromachining involves the deposition and patterning of surface layers such as metals, polysilicon or silicon oxynitrides on the surface of a wafer, which exists primarily to provide mechanical support. This process is closest to CMOS processes used for integrated circuits. Bulk micromachining refers to processes which modify the bulk of the wafer, typically by etching, or by bonding multiple wafers together.

Many devices are fabricated by a combination of surface and bulk micromachining processes. Recently a hybrid technique has emerged, based on silicon-on-insulator (SOI) wafers first developed for the electronics industry. A SOI wafer consists of a thin silicon device layer on a thin buried oxide layer on a standard silicon wafer. In CMOS processes a SOI wafer provides electrical isolation between components when the device layer is etched down to the buried oxide. In MEMS processes it provides a single-crystal silicon surface layer on a sacrificial oxide layer.

\subsubsection{Anisotropic Etching}

Micromachining etching processes are broadly categorized according to whether they are directional, wet versus dry, and their selectivity between different ma- 


\begin{tabular}{c|cc} 
& Isotropic & Anisotropic \\
\hline Wet & $\mathrm{HNO}_{3}+\mathrm{HF}(\mathrm{Si}), \mathrm{HF}$ (oxide) & $\mathrm{KOH}$ \\
Dry & $\mathrm{XeF}_{2}$ & DRIE
\end{tabular}

Table 3.1: Examples of etches that can be used for micromachining in silicon. A key advantage of wet etches is that they can be performed in standard laboratory glassware and require no special equipment. Deep Reactive Ion Etching (DRIE) can be used to produce highly anisotropic structures, however it requires processing equipment. Alkali solutions, such as potassium hydroxide $(\mathrm{KOH})$ stop on $\langle 111\rangle$ planes in silicon which allows the creation of etch pits with smooth side-walls, but is limited in the geometry of such structures compared to DRIE. For more details see [70, 69, 29].

terials. Selectivity refers to the ratio of the etch rates in two different materials, for example silicon and oxide. High selectivites are useful for stopping etches on a chosen layer or choosing a masking material for deep etches. Wet etches are those that have chemicals in aqueous solutions while dry etches are gaseous or plasma processes. Typically, dry etches require dedicated equipment while wet etches can be performed with standard laboratory glassware.

Anisotropic or directional etches are those that etch at different rates in different directions, while isotropic etches proceed at the same rate in all directions. Anisotropic etches are useful for defining features such as flat or vertical side-walls. Chemical etches which display anisotropic behavior in crystals typically do so along well-defined crystal planes. In single-crystal silicon this effect can be used to produce structures which are smooth down to nearly the atomic level.

The most common anisotropic wet etches for silicon are alkali or basic solutions such as potassium hydroxide $(\mathrm{KOH})$ and tetramethyl ammonium hydroxide (TMAH). These chemicals attack the $\langle 111\rangle$ crystal planes up to two orders of magnitude slower than either the $\langle 100\rangle$ or $\langle 110\rangle$ planes. In a $\langle 110\rangle$ wafer the $\langle 111\rangle$ planes form a $54^{\circ}$ angle with the surface which produces pyramidal pits. In a $\langle 110\rangle$ wafer there are $\langle 111\rangle$ planes perpendicular to the surface, allowing the fabrication of structures with straight and parallel vertical sidewalls. The two vertical $\langle 111\rangle$ planes form a $109^{\circ}$ angle and therefore cannot be used to create rectangular structures. Figure 3.1 shows the intersection of two $\langle 111\rangle$ planes on a $\langle 110\rangle$ surface produced by a $\mathrm{KOH}$ etch. 


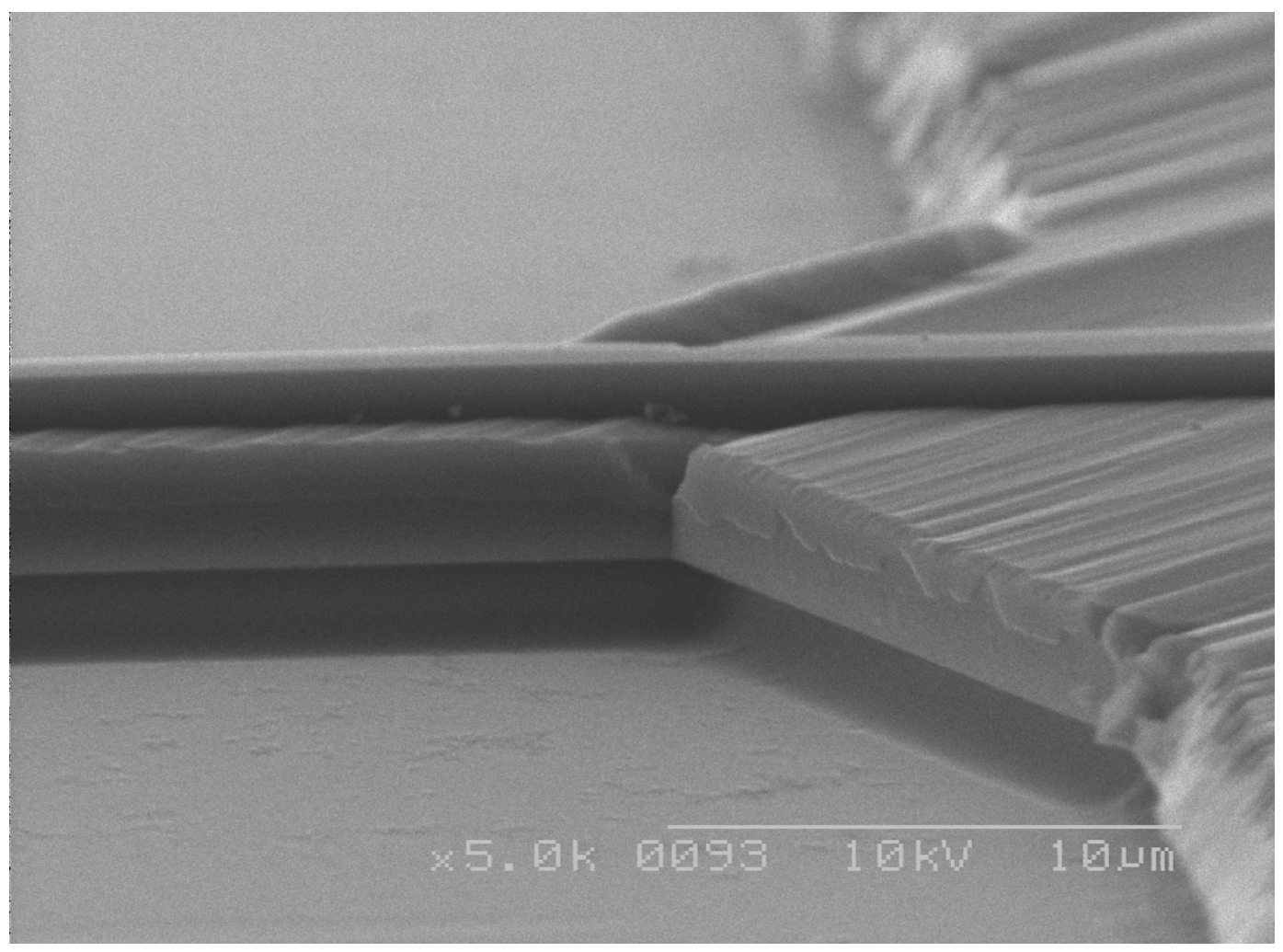

Figure 3.1: SEM micrograph showing a bridge and waveguide fabricated by anisotropic etching in potassium hydroxide.

\subsection{Device Design}

The simplest apparent geometry incorporating a curved moving waveguide is a simple beam, as illustrated in Figure 3.2. In the literature, a similar geometry has been demonstrated bending vertically [38] and in-plane [72]. In order to couple light into the beam one end must be anchored to allow the waveguide to continue on to the edge of the chip. The two options for the other end are free, as in a cantilever, or fixed, as in a bridge. Symmetry and the option to observe light passing through the device drive the choice of a bridge over a cantilever in this project.

In this work SOI was chosen as a substrate for device fabrication due to several key advantages. The mechanical properties of single-crystal silicon are well known and more predictable than deposited layers, which is an advantage for the resonator structure. Additionally, the device can be fabricated without the need to deposit mechanical or sacrificial layers, although several deposition steps are still required. Finally and most importantly, the fabrication of waveguides on SOI wafers is a well established technology which gives good results. 
Masters Thesis - Christopher Pope $\quad$ McMaster University - Eng. Physics

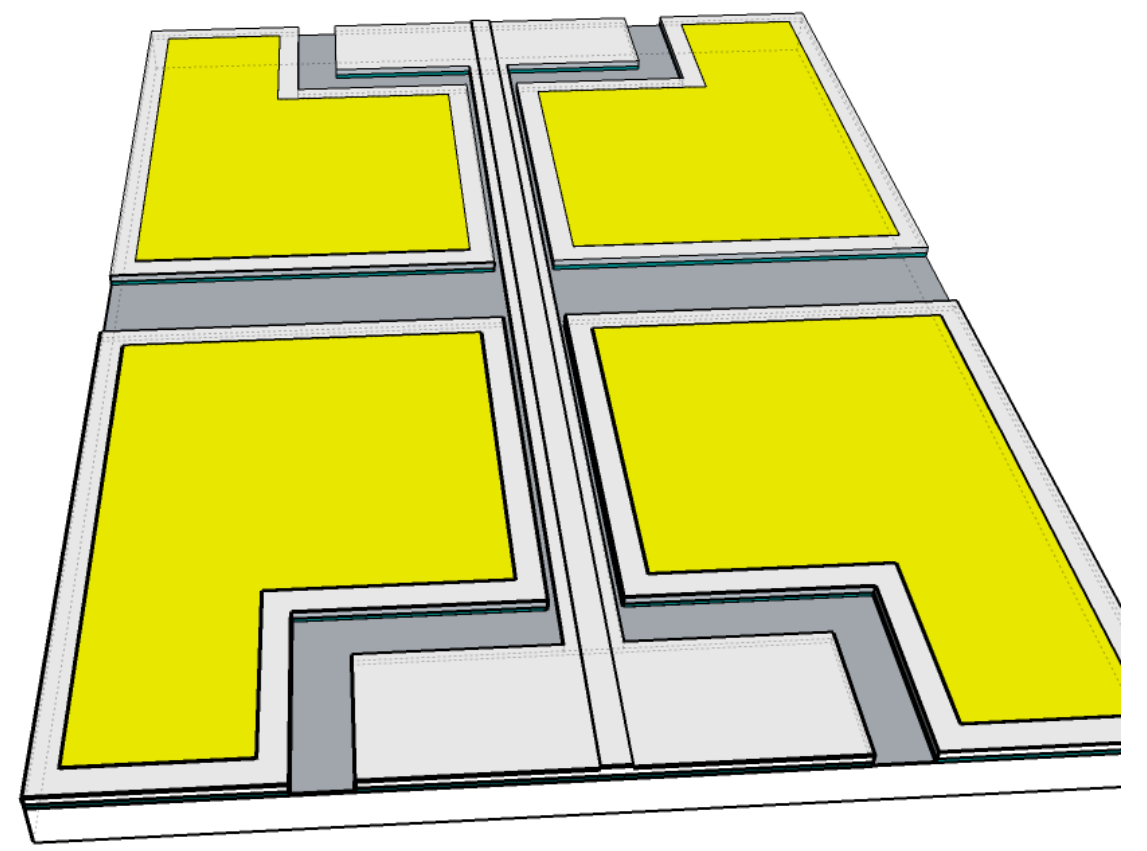

Figure 3.2: Rendering of the device design. Created in Google Sketchup [71].

Electrostatic techniques were chosen for both driving and detecting the motion of the beam. Capacitive detection is very sensitive while capacitive actuation is essentially the only technique available in this geometry. Electrodes are placed parallel to the bridge, with two electrodes on each side. This allows for a variety of electrostatic drive and detection options which are discussed in more detail in Section 4.1.1.

Optical confinement is obtained by fabricating rib waveguides of the type discussed in Section 2.4.2. Rib waveguides were chosen because of their good optical performance [64] and local experience with fabrication and experimental work [73]. To maximize the device sensitivity it was necessary to minimize the bridge and thus the waveguide width. To this end a waveguide width of $4 \mu \mathrm{m}$ was chosen, equal to the device layer thickness. Using the condition

$$
\frac{a}{b} \leq-0.05+\frac{r}{\sqrt{1-r^{2}}}
$$

we get a constraint

$$
\frac{21}{29} \leq r \leq 1
$$

on the height of the slab portion of the waveguide structure, to guarantee single-mode operation. The slab height should therefore be greater than $75 \%$ 
of the rib height. This corresponds to an etch depth of $1 \mu \mathrm{m}$, however, an etch depth of $800 \mathrm{~nm}$ is chosen to provide a safety margin. Polished waveguide end facets were chosen as the means to allow the introduction of light into the waveguides by fiber butt-coupling.

The remaining device dimensions were determined based on several constraints. The thickness of the structures is set by the $4 \mu \mathrm{m}$ device layer of the SOI wafers on-hand. Practical limitations in the photolithography process place a lower limit of $2 \mu \mathrm{m}$ on feature sizes. The bridge-electrode gap is thus chosen to be $3 \mu \mathrm{m}$ so as to provide some safety margin while maximizing sensitivity. A bridge width of $10 \mu \mathrm{m}$ was selected to provide the softest possible mechanical structure while still allowing the alignment and fabrication of a co-located waveguide.

Bridge lengths between $200 \mu \mathrm{m}$ and $1000 \mu \mathrm{m}$ were chosen to provide a variety of resonant frequencies. The longer bridges provide higher sensitivity by means of a lower spring constant, proportional to $1 / L^{3}$. An upper limit is placed on bridge length due to concerns about being able to fabricate and release such long, soft structures. The exact limit is not known for this geometry; a spread of lengths was chosen in the hope that shorter devices would survive if longer ones did not.

Five devices with bridge lengths stepped by $200 \mu \mathrm{m}$ were laid out on a chip to simplify processing and handling. This chip layout is illustrated in Figure 3.3. The chip is approximately $5 \mathrm{~mm}$ by $9 \mathrm{~mm}$, with the longer dimension chosen to fit in a $10 \mathrm{~mm}$ chip holder supplied with the critical point dryer. Eight of these chips, along with test structures and alignment markers were arranged on an approximately $25 \mathrm{~mm}$ square die. Mask fields were designed to enable the fabrication of this die as a whole. A drawing of the device is shown in Figure 3.4a.

\subsection{Fabrication}

The devices are fabricated on a commercially available SOI substrate. The device layer is $4 \mu \mathrm{m}$ thick and has a $\langle 110\rangle$ orientation. The buried oxide is $2 \mu \mathrm{m}$ thick and the handle wafer is $500 \mu \mathrm{m}$ thick with a $\langle 110\rangle$ orientation. The device layer is high resistivity, $>50 \Omega \mathrm{cm}$ to reduce optical absorption.

Broadly, the fabrication procedure consists of patterning the mechanical and optical structures, making electrical contacts, preparing devices and releasing the mechanical structures. The process has five photolithography steps, several wet etches, an ion implantation and a metal deposition. Photolithographic masks are illustrated in Figure 3.4 and cross-sections of the resulting device are shown in Figure 3.5 . 


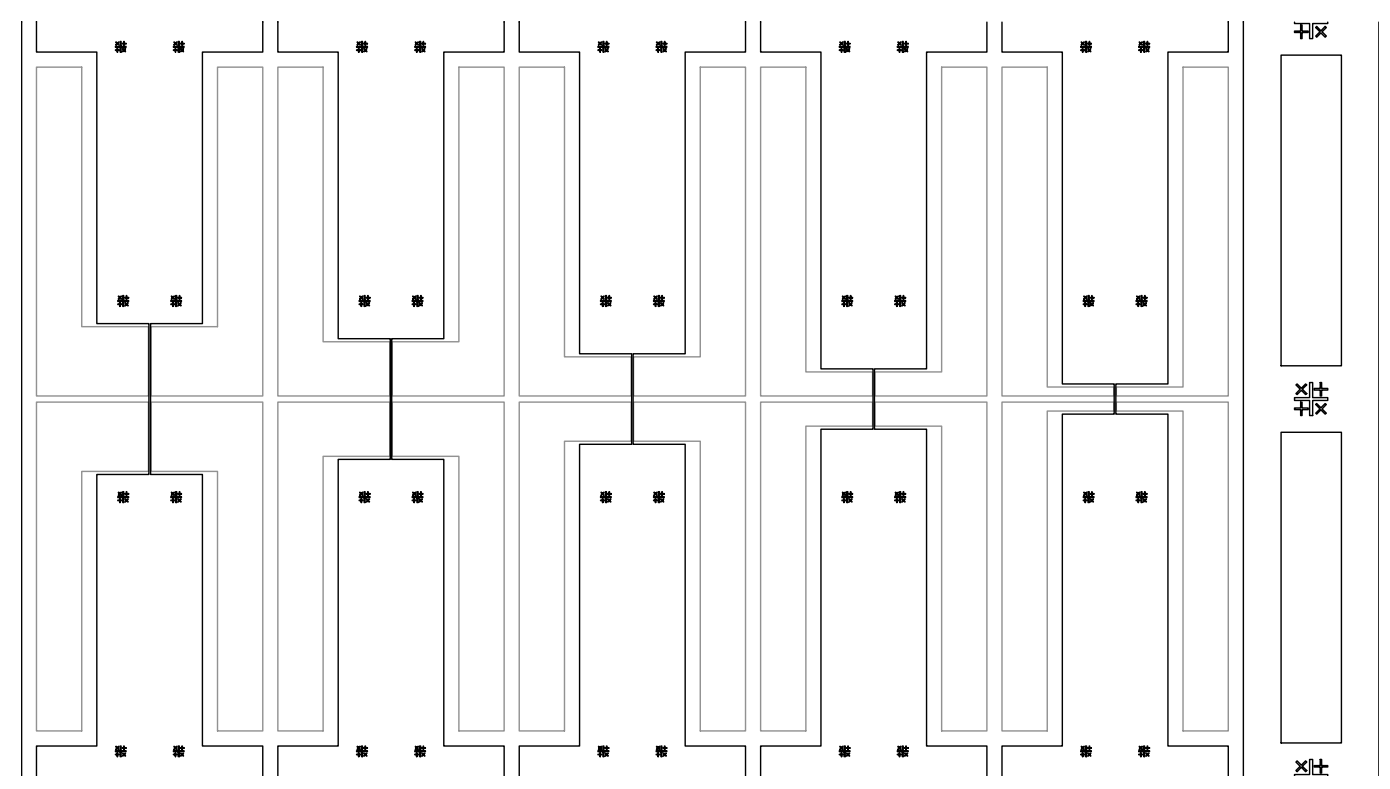

Figure 3.3: Section of the mask showing five bridges laid out on a chip. The pattern is repeated above and below for a total of four rows, and stepped to the left for a total of two columns and eight chips.

\subsubsection{Alignment}

The anisotropic etching of silicon in alkali solutions is sensitive to the exact alignment of the etch mask to the crystal planes in the sample. Misalignment will cause undercutting of the mask and may cause roughening of the sidewalls or distortion of the structure's shape. To improve pattern transfer from the mask to the sample it is thus beneficial to align to crystal planes in the sample with high accuracy. A procedure was developed to identify and align to the $\langle 111\rangle$ crystal plane in a $\langle 110\rangle$ silicon wafer.

This procedure was adapted from an example in literature [74, 75] and uses a single mask, shown in Figure 3.4b. The alignment target is two fan patterns on opposite sides of the sample, formed by line segments rotated in $0.1^{\circ}$ steps around a common point. When these lines are etched into the sample, the etch mask will be undercut where it is misaligned relative to the $\langle 111\rangle$ plane. The line which is undercut the least is therefore the best aligned to the crystal plane. A matching line on the next mask forms an alignment target which allows for accurate rotational alignment to the $\langle 111\rangle$ plane.

The alignment pattern is the first step performed. The pattern is transferred to the sample using the same process described below for the bridge and waveguide fabrication. Longer etch times, up to twice the thickness of the device layer, are used to increase undercutting and allow the alignment to be determined more easily. 


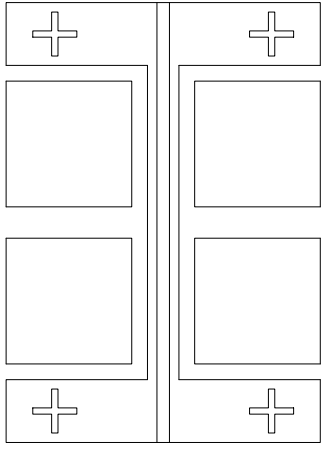

(a) Device layout

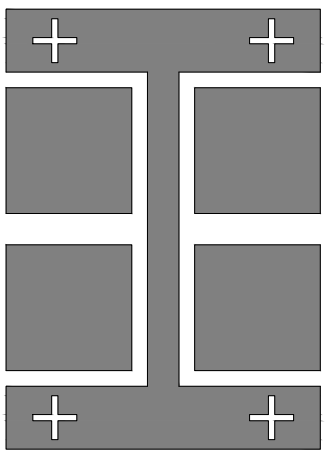

(c) Bridge mask

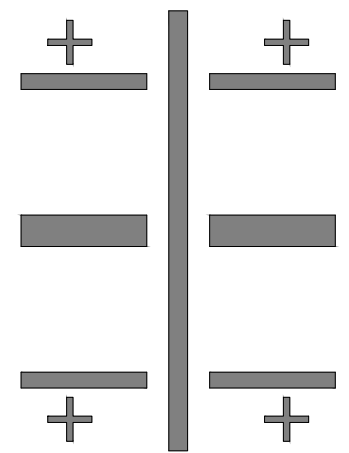

(e) Doping mask

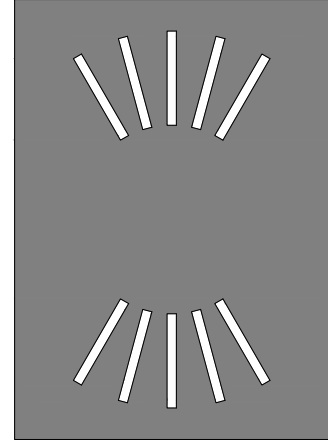

(b) Alignment mask

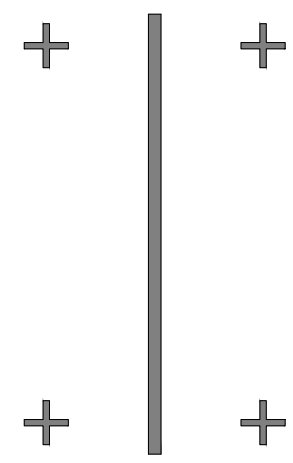

(d) Waveguide mask

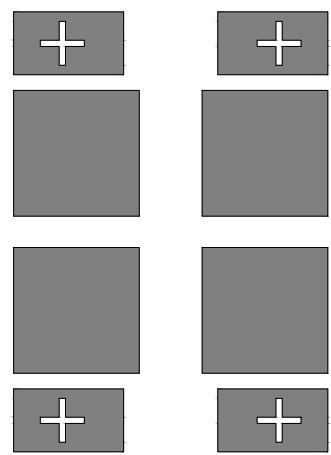

(f) Metalization mask

Figure 3.4: Simplified versions of the photolithography masks used in the fabrication process. Etch and doping masks are protected where dark. Metal mask deposits metal where dark. 
Masters Thesis - Christopher Pope McMaster University - Eng. Physics

\begin{tabular}{|c|}
\hline Si $(4 \mu \mathrm{m})$ \\
\hline $\mathrm{SiO}_{2}(2 \mu \mathrm{m})$ \\
\hline $\mathrm{Si}(500 \mu \mathrm{m})$ \\
\hline
\end{tabular}

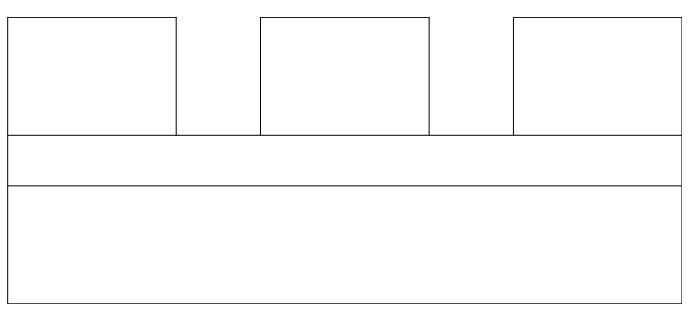

(a) Initial stack

(b) Bridge

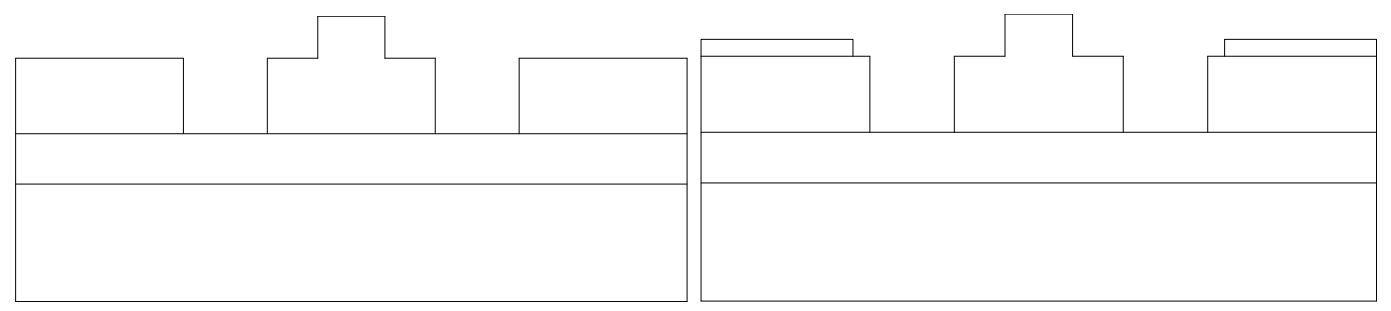

(c) Waveguide

(d) Electrodes

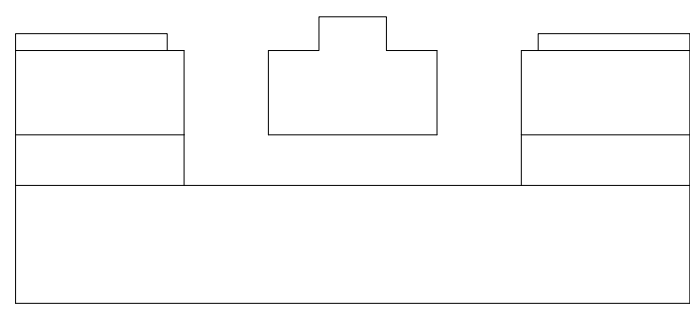

(e) Released

Figure 3.5: Diagram showing the device cross-sections after various process steps. 
McMaster University - Eng. Physics

\subsubsection{Bridges and Waveguides}

The bridge structures and waveguide ribs are defined with very similar processes, differing primarily in the pattern and the etch depth. The bridges and waveguides run parallel to the $\langle 111\rangle$ crystal planes, allowing the creation of smooth vertical sidewalls by means of an anisotropic potassium hydroxide (KOH) etch. The etchant is prepared as $40 \%$ weight $\mathrm{KOH}$ using the ratio of $80.5 \mathrm{~g}$ of $\mathrm{KOH}$ to $100 \mathrm{~mL}$ of water. The addition of $25 \mathrm{~mL}$ of isopropyl alcohol (IPA) reduces the surface tension of the solution to reduce bubble formation and surface roughness, but also changes the etch rate.

An oxide hard mask is used to mask the $\mathrm{KOH}$ etch. The oxide is grown by chemical vapour deposition (CVD) to a thickness of approximately $150 \mathrm{~nm}$. Silicon oxide etches significantly slower than silicon in $\mathrm{KOH}$ [70] and is thus a convenient mask. A photoresist layer was spun on on-top of the oxide to allow photolithographic patterning 1

The photoresist is patterned by contact photolithography using the pattern appropriate to the current process step (see Figure 3.4) and developed according to the manufacturer's instructions. The pattern is then transferred to the oxide mask by etching in buffered HF (BHF). Following this, the photoresist pattern is stripped using acetone. The silicon etch is performed in $40 \%$ weight $\mathrm{KOH}$ at $75^{\circ} \mathrm{C}$ based on a locally developed recipe [73]. The etch depth is confirmed by a surface profilometer (Tencor AlphaStep 200). Following successful completion of the etch the oxide hard mask is stripped in BHF.

The bridge etch is performed to the full thickness of the device layer, stopping on the buried oxide. The alignment marker etch is performed to the same depth and allowed to run longer to increase the degree of undercutting. The waveguide ribs are etched to a depth of $0.8 \mu \mathrm{m}$ to $1 \mu \mathrm{m}$, as discussed in Section 3.2 .

\subsubsection{Contacts}

The device layer has a low conductivity to reduce absorption by free carriers, but this is undesirable for the electrical performance of the device. In order to optimize both the electrical and optical properties of the device, a selective doping step was performed. The doping mask exposes the bridge and electrode sidewalls and the contact pads while protecting the waveguide.

A layer of photoresist is spun on, patterned with the doping mask (figure 3.4e and developed according the the manufacturer's instructions. Doping is performed by ion implantation of phosphorus at an energy of $15 \mathrm{keV}$ to a dose

\footnotetext{
${ }^{1} \mathrm{~A}$ negative photoresist (Futurrex NR9-1500P) is used for the alignment marker and bridge patterning steps while a positive photoresist (Shipley SC1827) is used for the waveguide, doping and metalization steps. The photoresist is chosen based on the mask tone and does not represent any fundamental requirement.
} 
Masters Thesis - Christopher Pope McMaster University - Eng. Physics

of $10^{15} \mathrm{~cm}^{-2}$. Following the implantation the photoresist was stripped using piranha etch. 2 The sample was then annealed at $1000{ }^{\circ} \mathrm{C}$ for 15 minutes to diffuse and activate the dopants.

Metal contact pads are added to allow electrical contact to easily be made to the device. The contacts were formed by depositing a $20 \mathrm{~nm}$ chrome adhesion layer followed by a $200 \mathrm{~nm}$ gold contact layer. The metals were deposited in an ultra-high vacuum electron beam evaporation system. The contacts were patterned by a lift-off process, which begins by applying and patterning a photoresist layer (using the mask in Figure 3.4f). In this process the photoresist is specially developed to induce undercutting of the side-walls. The metal is deposited on top of the photoresist in areas where it is not wanted, and lifts off in an acetone bath. The completed device at this stage is shown in Figure 3.6.

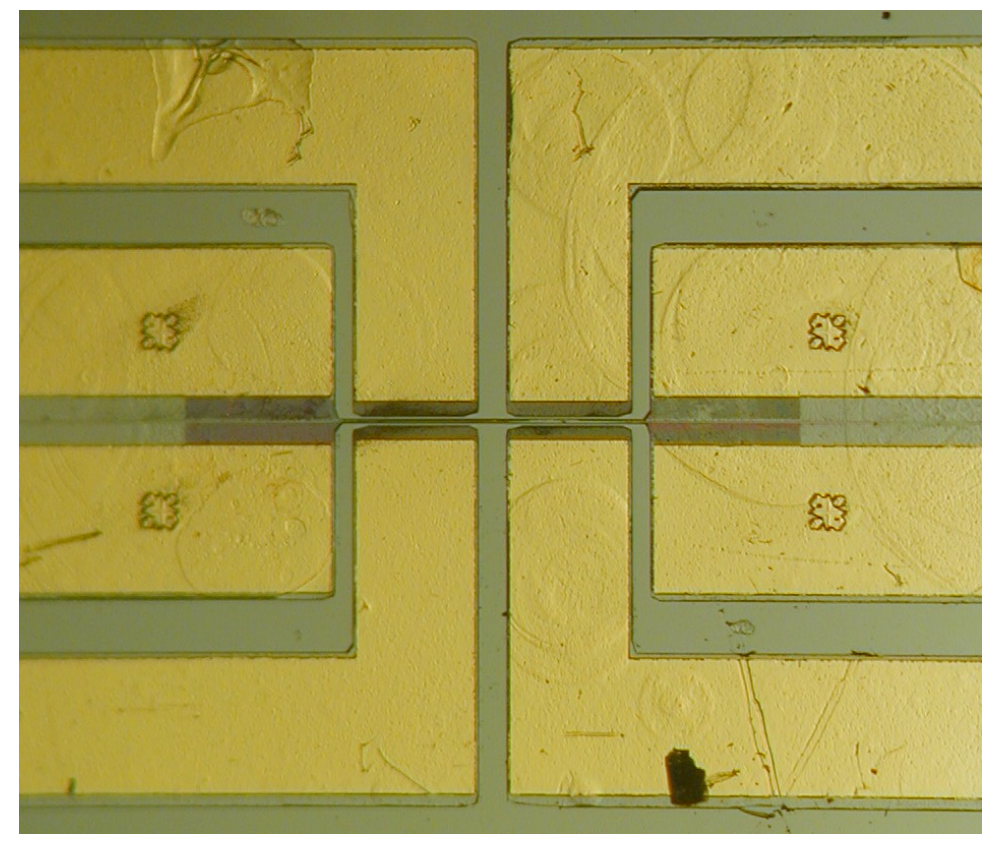

Figure 3.6: Micrograph of a fabricated $600 \mu \mathrm{m}$ bridge before release.

\subsubsection{Release}

The devices are fabricated in an array with five devices on a chip and eight chips on the sample. Separation of the individual chips is done by scribing and cleaving. This process is violent and dirty and is thus best performed before releasing the mechanical structures. There is no cleavage plane in the device

\footnotetext{
${ }^{2}$ Ion bombardment can cause significant hardening of photoresists. Aggressive measures such as pirahna etch may be required to remove resists hardened in this way.
} 
layer perpendicular to the waveguides, so some polishing may be required to get facets of optical quality.

Releasing of the mechanical structures is performed by etching the oxide layer in $49 \% \mathrm{HF}$ to undercut the bridges, followed by processing in a critical point dryer (Tousimis Autosamdri-815 Series B). Critical point drying was used to eliminate surface tension during the drying process which has the potential to destroy structures in this size range. In this process the sample is rinsed in DI water following the HF etch and then transferred to a bath of isopropyl alcohol ${ }^{3}$ without allowing the sample to dry. Water is displaced by the alcohol, and the sample is transferred to the critical point dryer under liquid. Liquid carbon dioxide $\left(\mathrm{LCO}_{2}\right)$ is the working fluid in the critical point dryer. The dryer purges the alcohol from the chamber and carries out the drying cycle automatically.

By raising the temperature and pressure, the $\mathrm{CO}_{2}$ is taken past its critical point $\left(31^{\circ} \mathrm{C}\right.$ and $7.4 \mathrm{MPa}$ for $\left.\mathrm{CO}_{2}\right)$. Controlled decompression allows the $\mathrm{CO}_{2}$ to be exhausted as a gas. Because the gas and liquid phases cease to be distinct past the critical point (where the gas-liquid phase transition curve ends) the sample can be dried without the liquid experiencing a phase transition. Thus, there is no gas-liquid interface to create surface tension which could damage the structures. The structure released by these techniques is shown in Figure 3.7. where the undercut bridge is visible.

In order to perform electrical tests, the chips are mounted to customdesigned printed circuit boards (PCBs). PCBs were found to provide superior electrical performance and repeatability when compared to alternatives such as mounting to DIP headers. The chips were mounted using silver paint. Electrical connections were made by wire-bonding to the PCB using gold wire and a ball bonder (Kulicke \& Soffa 4522). Details of the electrical connections will be discussed in Section 4.1.1.

\subsection{Fabrication challenges}

\subsubsection{Facet quality}

As discussed above, coupling of light into the waveguides is to be accomplished by means of butt-coupling of a cleaved fiber to the waveguide end-facet. Maximizing the coupled power requires that the waveguide facet be smooth and clean to minimize scattering. Typically this is accomplished by cleaving, however this proves to be difficult in this case. Because the waveguides are parallel to the $\langle 111\rangle$ plane in the $\langle 110\rangle$ device layer there is no cleavage plane perpen-

\footnotetext{
${ }^{3}$ Isopropyl alcohol is used at the manufacturer's recommendation. IPA is miscible with both water and $\mathrm{LCO}_{2}$, allowing the water to be completely eliminated.
} 


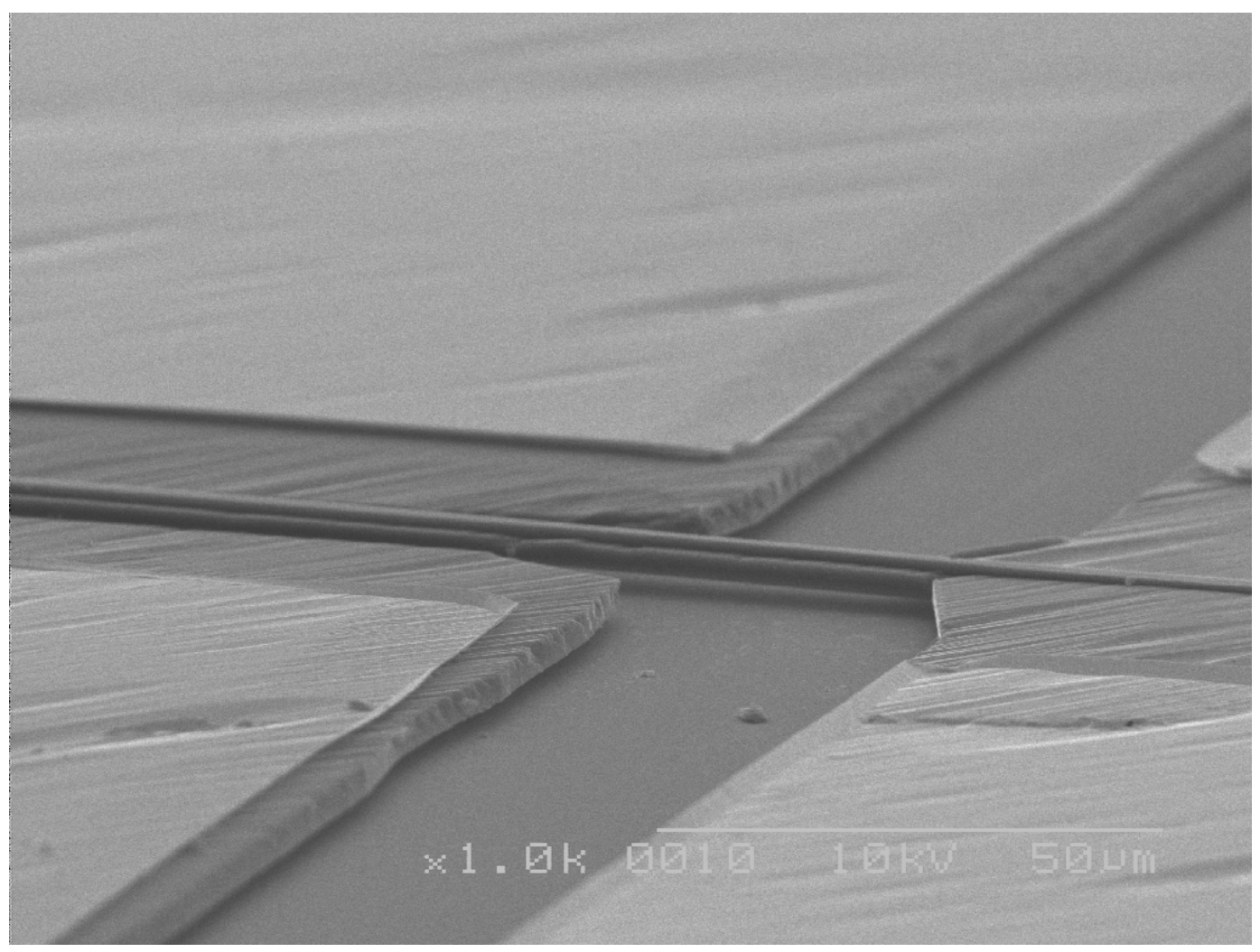

Figure 3.7: SEM micrograph of a fabricated and released bridge.

dicular to the waveguide. A further complication is that the thickness of the handle wafer is, at $500 \mu \mathrm{m}$ quite thick and difficult to cleave. Finally, while it is the device layer that must cleave cleanly to produce a facet, it is the handle wafer that provides the bulk of the mechanical strength and thus dominates the cleavage behavior.

In the first fabrication batch, the device-bearing chips were separated by first scribing along scribe marks on the sample and then cleaving along the scribed line. This was successful at separating the chips but did not produce a high-quality facet. A SEM image of an edge of a typical chip is presented in Figure 3.8 showing the roughness left by the scribing and cleaving process. The illustrated waveguide facet is completely unsuitable for optical coupling.

A simple resolution to this issue would be to polish the facet, as the process for doing so is well understood. Polishing must be done prior to releasing the mechanical structure or else there is a risk of damaging the delicate bridges. An alternate solution would be to use a dicing saw to separate the chips. The quality of the resulting facets is uncertain and it shares at minimum the same damage risks as polishing. A long-term option is to redesign the device to use grating couplers [76] which allow coupling light into the surface of the 


Masters Thesis - Christopher Pope McMaster University - Eng. Physics

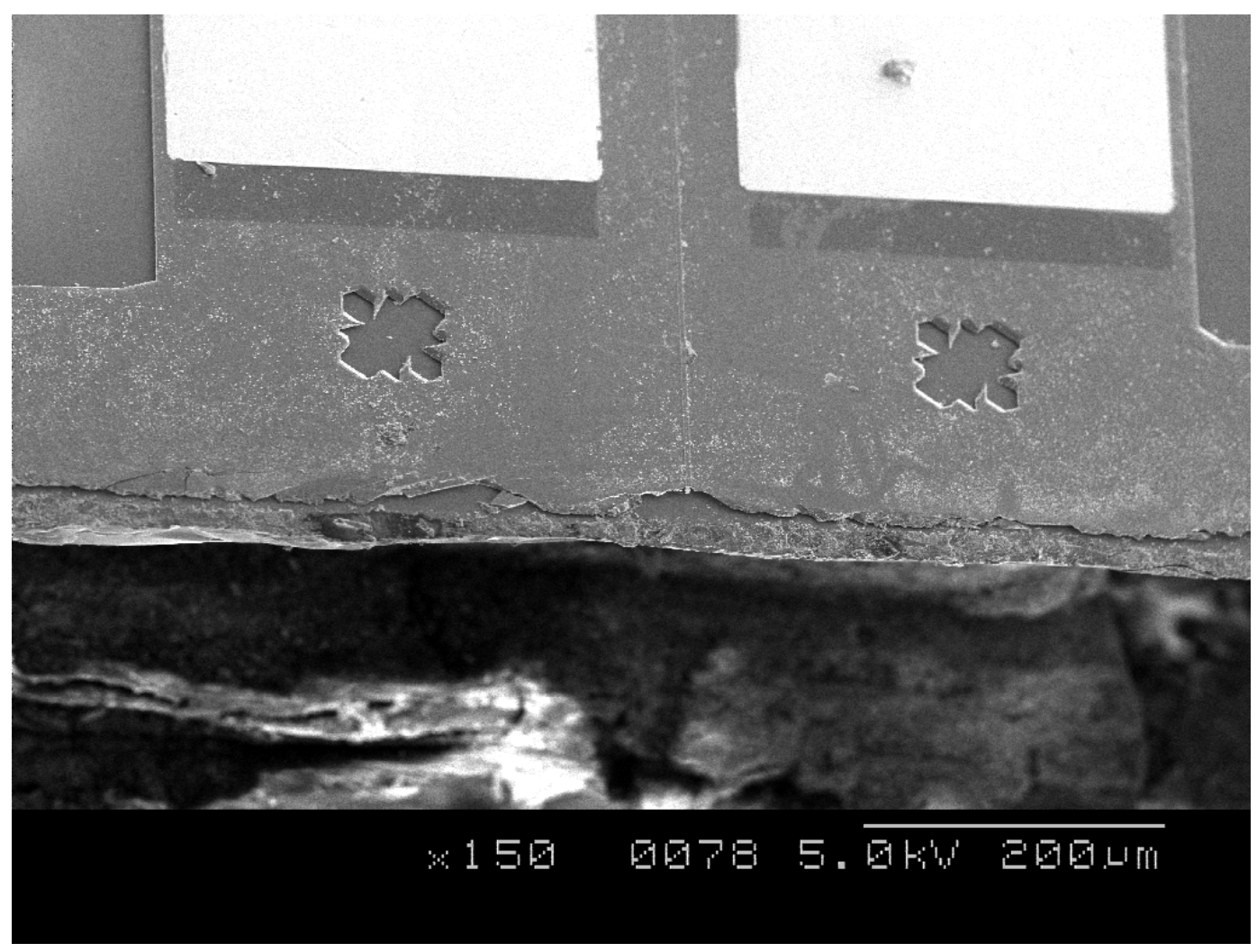

Figure 3.8: SEM micrograph showing the cleaved edge of a device and the resulting damage to the device layer.

waveguide. While a grating coupler eliminates concerns about facet quality (by eliminating their use entirely) it requires more from the fabrication process. As an optically resonant structure, a grating coupler requires features on the order of the optical wavelength which would be difficult to obtain in the current manufacturing process.

\subsubsection{Bridge curvature}

Upon releasing devices from the first fabrication run it was discovered that the longer bridges were curved up out of the plane, as shown in Figure 3.9. While not significant at shorter lengths, the curvature is enough to raise the $1000 \mu \mathrm{m}$ bridge substantially away from its electrodes. This is a cause for concern as it decreases the bridge-electrode capacitance due to the decrease in overlap area. A mitigating factor is that in the proposed experiment design there exists an electrostatic potential between the bridge and the substrate which would oppose and possibly correct this curvature.

While the cause of this curvature is unknown, it is believed to be due to the post-implant anneal process performed in the doping step. The thermal 


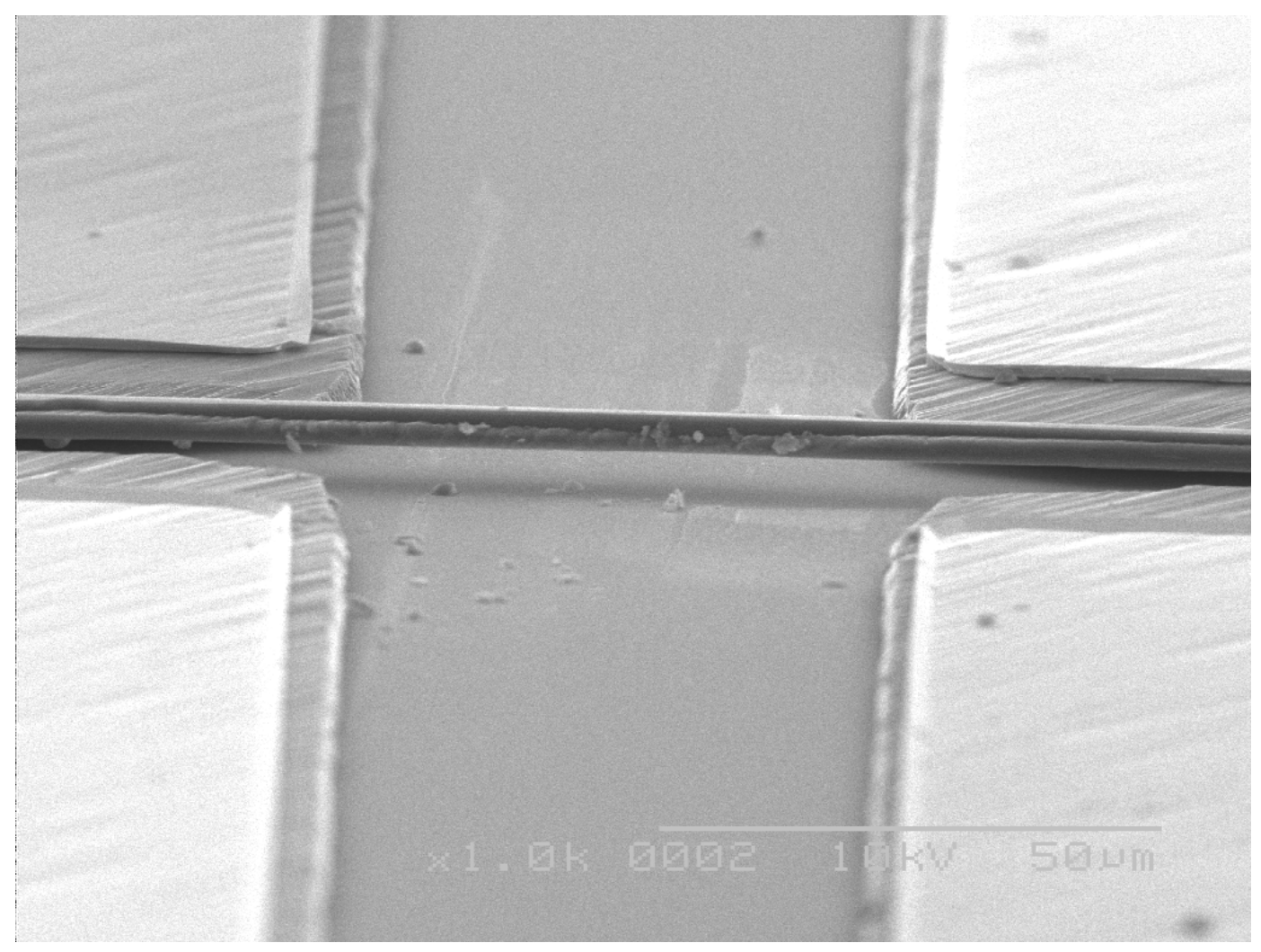

Figure 3.9: SEM micrograph showing a released $1000 \mu \mathrm{m}$ bridge curved up out of the plane of the device layer.

expansion coefficients of silicon and the underlying oxide are different 4 , which would lead to a strain buildup at the annealing temperature of $1000^{\circ} \mathrm{C}$. If any of this strain was relaxed while at this temperature there would be a residual strain when the structure is cooled. Such an effect is mentioned in the user's guide for the commercial SOIMUMPs process [78], which recommends a maximum length of $500 \mu \mathrm{m}$ for $6 \mu \mathrm{m}$ wide structures anchored at both ends.

Because silicon expands and contracts faster than the oxide, the built-in strain after a relaxation at high temperatures will place the silicon side of the interface in tension and the oxide side in compression. When the oxide is etched away during the device release step, the silicon layer will be free to move and contract at the former interface in order to relieve the built-in strain. This causes the upward deformation of the bridge into its fundamental mode, where the middle half of the bridge is in tension on top and compression on the bottom.

\footnotetext{
${ }^{4}$ Fused quartz has a thermal expansion coefficient of $0.55 \times 10^{-6} \mathrm{~K}^{-1}$ while for silicon it is $2.6 \times 10^{-6} \mathrm{~K}^{-1}$ [7]
} 
Masters Thesis - Christopher Pope McMaster University - Eng. Physics

The maximum value for the strain in the silicon layer is

$$
\epsilon=\left(\alpha_{S i}-\alpha_{S i O_{2}}\right) \Delta T
$$

which is equal to $-2.05 \times 10^{-3}$ if the structure is completely relaxed at the annealing temperature. 


\section{Chapter 4}

\section{Experiment}

\subsection{Electrical Experiment}

\subsubsection{Electrical connections}

Devices were fabricated following the procedure outlined in Section 3.3. The resulting device is shown in Figure 3.6. There are nine electrical connections: four electrodes, four (theoretically identical) bridge contacts and the substrate. Figure 4.1 shows the six fundamental terminals in relation to the capacitances of interest. Not shown are parasitic capacitors formed between adjacent electrodes and between each electrode and the substrate. These parasitics are potentially quite significant as they are orders of magnitude larger than the working capacitances due to the larger areas involved. Proper measurement technique can reduce the impact of this issue.

Several methods exist to connect the device to laboratory test and measurement equipment. Three methods that were explored are probes, DIP header mounting and PCB mounting. The key evaluation criteria is electrical performance, requiring low parasitics, low noise and repeatable results. Mechanical stability and ease of use also played a role in the evaluation.

Probes for making contact to integrated circuits are commonly available commercially, and some varieties can offer good performance. Connections to several devices were made using a commercially available two-terminal probe station and capacitance measurements were made successfully. While this method offers a simple way to test the electrical properties of the device, several issues were identified which made it unsuitable for further experiments. The probe tips in this setup were unshielded and therefore had significant mutual capacitance, larger than the working capacitance of the device. This can be seen simply by making consecutive measurements of the device and then lifting the probes slightly from the surface. Additionally, the system is large and expensive compared to other options and lacks the mechanical integrity that 
Masters Thesis - Christopher Pope $\quad$ McMaster University - Eng. Physics

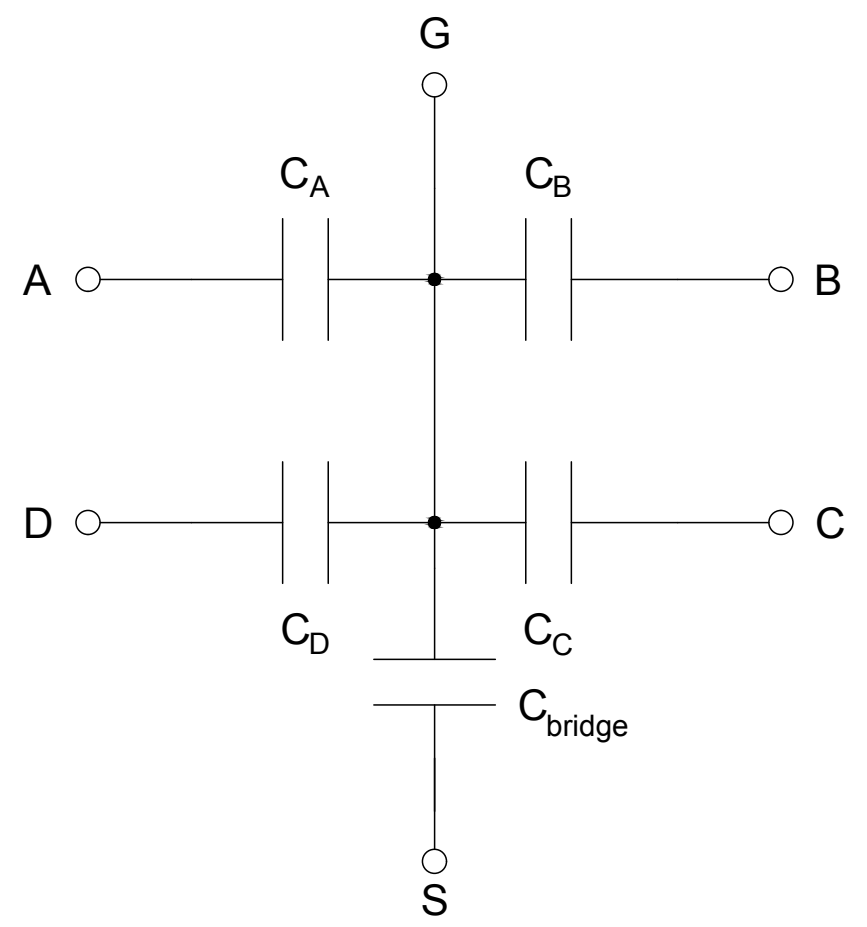

Figure 4.1: Schematic drawing showing the terminals available for making connection to the bridge resonator. The capacitors shown are formed between the bridge (terminal $G$ ) and the drive/detect electrodes. The capacitor $C_{b r i d g e}$ is formed between the bridge and the substrate (terminal $S$ ). Not shown are various parasitic capacitances.

is desired for long-term measurements.

A second option was to use a standard Dual Inline Pin (DIP) package of the type commonly used for integrated circuits commercially. Previous work locally has advanced this method for testing MEMS devices [79]. This was explored by mounting a device to a 20-pin metal DIP package using conductive silver paint. Electrical connections were made by wire-bonding from the device contact pads to the package pins, using gold wire and an ultrasonic ball bonder. This method, standard practice in the IC industry, was found to require some skill but produces reasonably durable connections.

The DIP package was placed inside a metal box which acted as a Faraday cage. Short wires were soldered between the DIP package pins and BNC connectors on the box, allowing the attachment of standard coaxial cables. Measurements were made successfully in this setup, however it was not without problems. The hook-up wires used to connect the device to the cables showed significant mutual capacitance, at lengths of approximately $5 \mathrm{~cm}$. This capacitive coupling allowed significant amounts of drive signal to contaminate the output signal which complicated measurements. 
To some extent this coupling can be reduced by replacing the hook-up wire with coaxial cable. Improvements due to the shielding effect of the coaxial cable's grounded shield were shown experimentally. Soldering connections to the stiffer shielded cable was difficult in the confinement of the Faraday cage. One connection was made successfully, however the difficulty increased with additional connections. While these problems were potentially resolvable through a redesigned enclosure, when combined with ongoing concerns about mechanical reliability it was decided to investigate a simpler and cheaper alternative. This investigation began with printed circuit boards, based partly on their use in a recent paper [80].

Products from the cheapest toys to complex microwave circuits bond semiconductor devices directly to circuit boards for reasons of cost and electrical performance. The motivation in this case was the ability to effectively shield traces on the PCB by means of a large ground plane, reducing issues that affected the alternative methods described above. In addition, standard PCB-mount coaxial connectors could be used to make completely shielded connections to measurement equipment.

Custom PCBs were designed based on the above goals. The second revision of the board is shown in Figure 4.2. The device was mounted to the large pad, with three smaller pads provided on each side for connecting electrodes and the bridge. All electrical connections are routed to SMA connectors allowing the attachment of coaxial cables. The board size is only $50 \mathrm{~mm}$ square, limited by mounting holes and the minimum spacing required by the connectors.

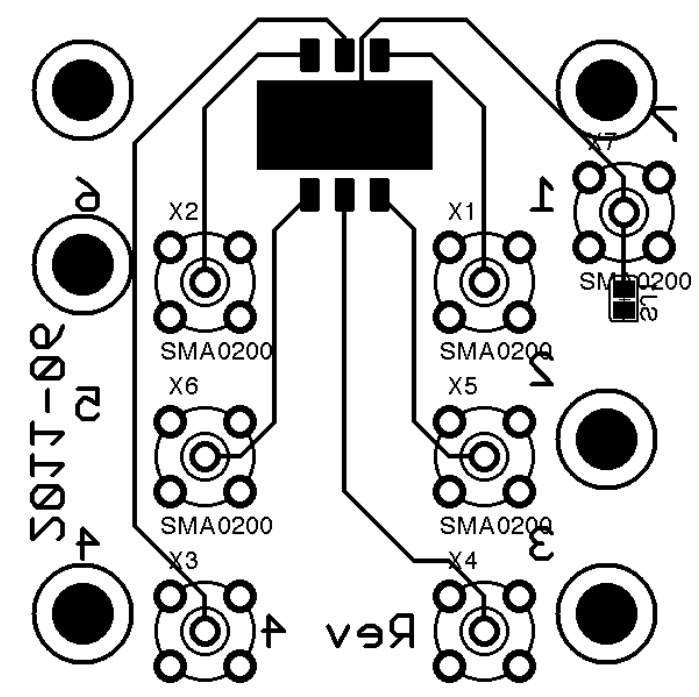

Figure 4.2: Design of the PCB on which devices were mounted and tested. Finished board size is two inches square and contained a full ground-plane. The board was designed using EagleCAD [81]. 
Inspiration was taken from PCB design rules for high-speed electronic circuits, in particular with respect to reducing capacitance issues, although the operating frequencies here are low by modern standards [82, 83]. Signal traces are kept as short as possible and well-seperated to reduce mutual capacitance. The entirety of the board surface area, except where signal traces are routed, forms a single ground plane which is connected to the shields of the SMA connectors. Six mounting holes are provided on 0.5 inch centers to accept \#832 screws, which offers compatibility with standard optical components. The off-center device mounting position is influenced by the needs of the optical experiment described in Section 4.4. A photo of the finished PCB with device mounted is shown in Figure 4.3 .

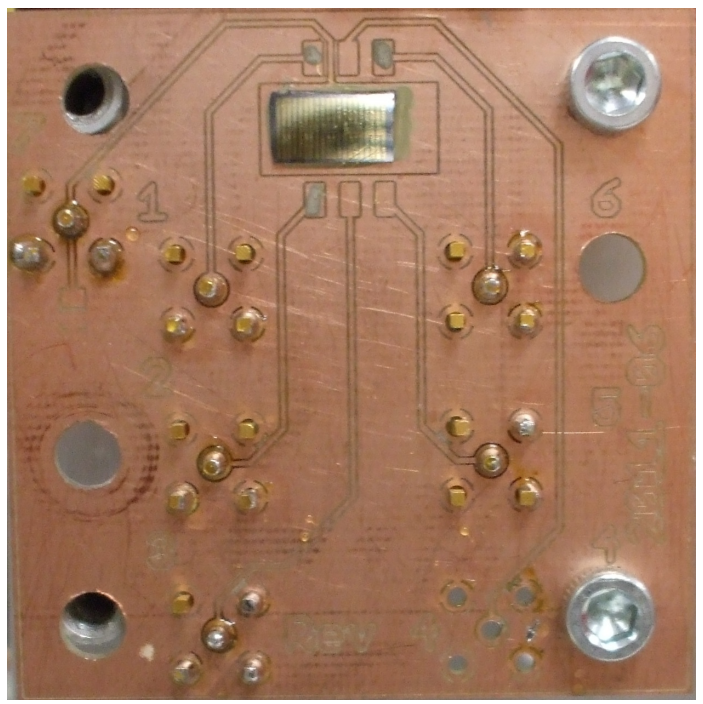

Figure 4.3: Photograph of a completed PCB with a device mounted and ready for testing.

\subsubsection{Capacitance measurements}

Initial capacitance measurements were performed with an Andeen-Hagerling AH 2500A automatic capacitance bridge ${ }^{1}$ This instrument allows measurement of both the capacitance and loss or parallel conductance of the device under test (DUT). In order to reduce the effects of parasitic capacitances which may appear between the terminals of the DUT, or be contributed by cables and test fixtures, the AH2500 makes three-terminal measurements.

The three-terminal measurement technique, illustrated in Figure 4.4, involves ensuring that all spurious or parasitic capacitances are grounded, with

\footnotetext{
${ }^{1}$ The manual for this instrument provides an excellent overview of the techniques of high-precision capacitance measurements 84.
} 
this ground connection forming the third terminal 2 This is typically accomplished by grounding all nearby conductive objects or placing the DUT in a metal box. An added advantage is that such an enclosure forms a Faraday cage, isolating the system from RF interference.

The three terminals used to make a simple capacitance measurement are the excitation (high) terminal, the detection (low) terminal and the ground terminal. When an AC test voltage is applied to the $H$ terminal it also appears across the parasitic capacitor $C_{H G}$, which has no effect on the current through the DUT (provided the total current does not exceed the current compliance of the source). The input of the amplifier at the $L$ terminal is a virtual ground and thus collects essentially all the current flowing through the DUT, which bypasses the higher impedance of $C_{L G}$. In this way the measurement of the capacitance $C_{H L}$ is unaffected by the presence of other parasitic capacitances.

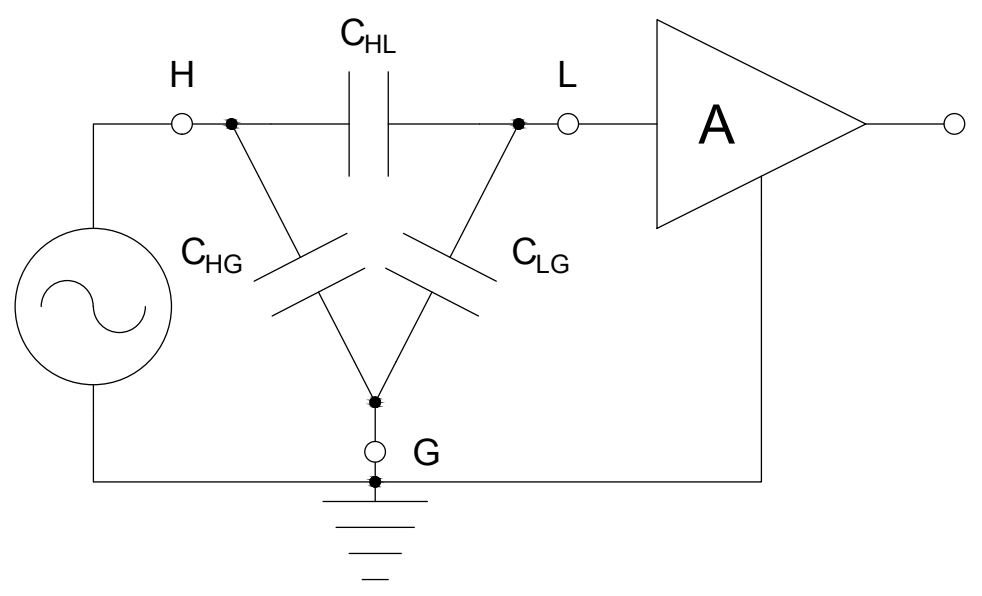

Figure 4.4: Schematic drawing of a three-terminal measurement circuit.

Two measured capacitance values are of interest in characterizing the device. The first is the capacitance measured between the bridge and the electrode, which is the working capacitance responsible for the device motion and the electrical response. The second is the capacitance between two electrodes, which is representative of the parasitic coupling between the drive and detect electrodes. The device design attempts to reduce this parasitic by spacing

\footnotetext{
${ }^{2}$ Capacitance measurements may be made with between two and five terminals, depending on requirements. Two-terminal measurements simply use the current flowing between the instrument terminals to determine the capacitance. Three-terminal measurements, as described here, improve accuracy and noise immunity. Four-terminal measurements follow the example of four-terminal resistance measurements where separate conductor pairs are used to apply the excitation and make the measurement. This allows for improved accuracy where losses in the connecting cables may be significant but is typically not applicable to these small capacitances and distances. A five-terminal measurement combines the features of three and four-terminal measurements.
} 
Masters Thesis - Christopher Pope

McMaster University - Eng. Physics

\begin{tabular}{cccc}
$\begin{array}{c}\text { Length } \\
(\mu \mathrm{m})\end{array}$ & $\begin{array}{c}\text { Bridge-Electrode } \\
\text { Capacitance }(\mathrm{fF})\end{array}$ & $\begin{array}{c}\text { Bridge-Substrate } \\
(\mathrm{pF})\end{array}$ \\
\hline & & 8.9 & \\
200 & 0.53 & 1.8 & 6.3 \\
400 & 1.4 & 2.7 & 6.7 \\
600 & 2.3 & 3.5 & 7.2 \\
800 & 3.2 & 4.4 & 7.6 \\
1000 & 4.1 & 8.0 \\
\hline
\end{tabular}

Table 4.1: Calculated device capacitances based on the parallel plate approximation. This approximation neglects the effects of fringing fields.

electrodes farther apart $(40 \mu \mathrm{m})$ than the working capacitor gap of $3 \mu \mathrm{m}$. Experimental design attempts to reduce the parasitic coupling by grounding unused electrodes and the substrate, and by making (effectively) three-terminal measurements with the bridge as a virtual ground.

Table 4.1 lists theoretical values for the bridge to electrode working capacitance, the bridge to substrate capacitance and the capacitance between the electrode pad and the substrate for all five fabricated bridge lengths. It is of interest that the parasitic capacitance between the electrode and the substrate is three orders of magnitude larger than the working capacitances. These values are calculated using the parallel plate approximation (equation (2.114)) and do not take into account the contributions from fringing fields at the edges of the capacitor plate.

Measured values for the two device capacitances of interest are summarized in Table 4.2. The reported values are measured between two terminals of the device mounting system and referenced to a local ground plane appropriate for the mounting system. Offsets from the capacitance bridge and connecting cables are negligible. Reported values are significantly higher than the estimates reported in Table 4.1 due to contributions from fringing fields and the mounting system. In particular, adjacent bond wires may contribute on the order of $10 \mathrm{fF}$, and the printed circuit board contributes approximately $12 \mathrm{fF}$ between adjacent pads (but only $0.5 \mathrm{fF}$ between non-adjacent pads).

\subsubsection{Experimental setup}

A basic measurement circuit for the bridge resonator is illustrated in Figure 4.5. This circuit is the combination of an actuation circuit using capacitor $C_{a}$ and a sensing circuit using capacitor $C_{s}$. These two components operate and can be analyzed independently. 
Masters Thesis - Christopher Pope $\quad$ McMaster University - Eng. Physics

\begin{tabular}{lccc} 
Setup & $\begin{array}{c}\text { Length } \\
(\mu \mathrm{m})\end{array}$ & $\begin{array}{c}\text { Bridge-Electrode } \\
\text { Capacitance }(\mathrm{fF})\end{array}$ & $\begin{array}{c}\text { Electrode-Electrode } \\
\text { Cander (floating) }\end{array}$ \\
600 & $12000 \pm 1000$ & $10000 \pm 1000$ \\
DIP Header & 600 & $250 \pm 75$ & $100 \pm 50$ \\
DIP Header (grounded) & 600 & $30 \pm 10$ & $20 \pm 10$ \\
DIP Header (shielded) & 400 & $60 \pm 10$ & $10 \pm 5$ \\
DIP Header (shielded) & 600 & $150 \pm 50$ & - \\
PCB Mount & 1000 & $150 \pm 50$ & $20 \pm 5$ \\
PCB Mount & & & \\
\hline
\end{tabular}

Table 4.2: Summary of measured capacitance values for several device mounting options. All measurements were taken using the AH2500A capacitance bridge. Error estimates represent fluctuations in measured values and pad-topad variations rather than instrument limitations. Devices mounted to DIP headers were measured with the substrate floating and grounded, and with a coaxial cable used in place of one hook-up wire.

Actuation of the device is done using an actuation voltage $V_{A C}=v \sin \omega t$ combined with the bias voltage $V_{D C}$ applied across the capacitor $C_{a}$. Based on the result from Equation (2.121) there will be forces on the bridge which are periodic and frequencies $\omega$ and $2 \omega$. As the bridge is a resonant structure it will respond most strongly when the force is applied at the mechanical resonance frequency $f_{r}$. This results in a motion of the bridge which can be measured.

As the bridge moves. the capacitance $C_{s}$ changes, and by Equation (2.116) there is a current

$$
i=V \frac{d C}{d t}=V_{D C} \frac{d C_{s}}{d x} \frac{d x}{d t}
$$

through the capacitor which is proportional to the velocity of the bridge. At resonance the motion of the bridge is periodic with frequency $f_{r}$ and likewise the current is periodic. This current flows into the charge-sensitive amplifier, or rather though the feedback capacitor $C_{f}$. The output voltage is

$$
V_{\text {out }}=-i Z_{f}=\frac{-V_{D C}}{\omega C_{f}} \frac{d C_{s}}{d x} \frac{d x}{d t}
$$

which is independent of the drive frequency $\omega$ if the motion of the bridge is sinusoidal. This may be contrasted with a transimpedance amplifier $\left(Z_{f}=R_{f}\right)$ where the output voltage is proportional to the actuation frequency.

An overview of the experimental setup is in Figure 4.6 which shows the equipment used for determining resonant frequencies. All signal connections 


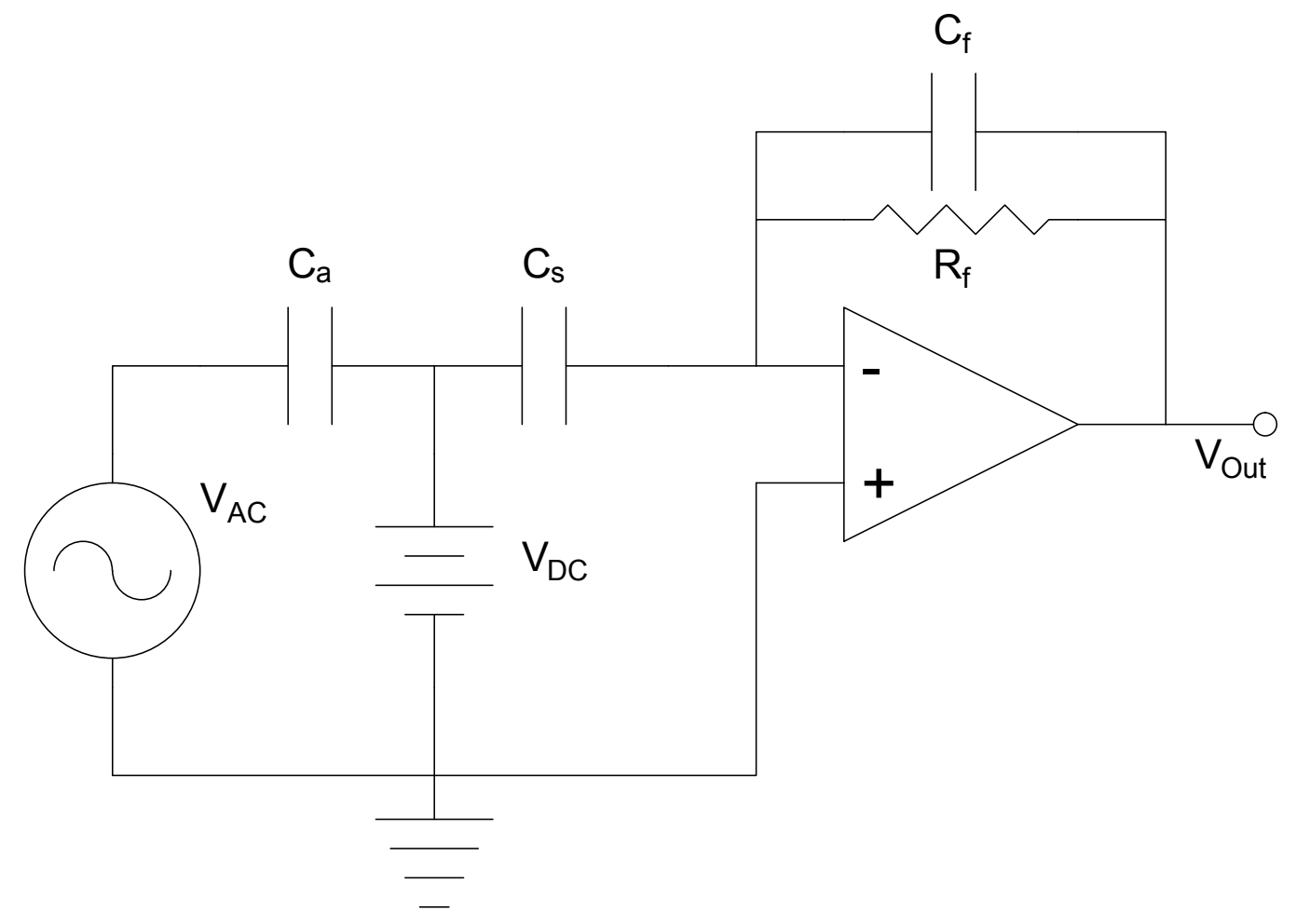

Figure 4.5: Schematic drawing of the basic drive/detect circuit.

are made using coaxial cables with the shields grounded. Avoiding coupling between the drive and detect electronics was a major goal, accomplished largely though careful grounding of stray capacitance. To this end, all unused device terminals were grounded, including the substrate and the ground plane of the $\mathrm{PCB}$, in an attempt to take advantage of the three-terminal measurement technique discussed above.

Actuation is accomplished by means of a sinusoidal drive signal provided by a Hewlett Packard (HP) 33120A function generator ${ }^{3}$ Bias voltages were provided by an HP 3245A universal source or a HP 6212B linear power supply. The HP 3245A has an output range of $\pm 10 \mathrm{~V}$, however this range can be offset from $0 \mathrm{~V}$ by stacking its output in series with the HP $6212 \mathrm{~B}$ as the latter has floating outputs and a $100 \mathrm{~V}$ output range.

Sensing the device response is done through an Amptek A250 charge sensitive preamplifier [85]. This amplifier is chosen for its low noise performance and performs current to voltage conversion. Output from the amplifier feeds a Stanford Research Systems SR844 lock-in amplifier which measures the device

\footnotetext{
${ }^{3}$ The Hewlett Packard (HP) equipment used in these experiments was manufactured prior to the spin-off of HP's instrument division as Agilent Technologies in 1999. Manuals are available from Agilent via http://agilent.com
} 


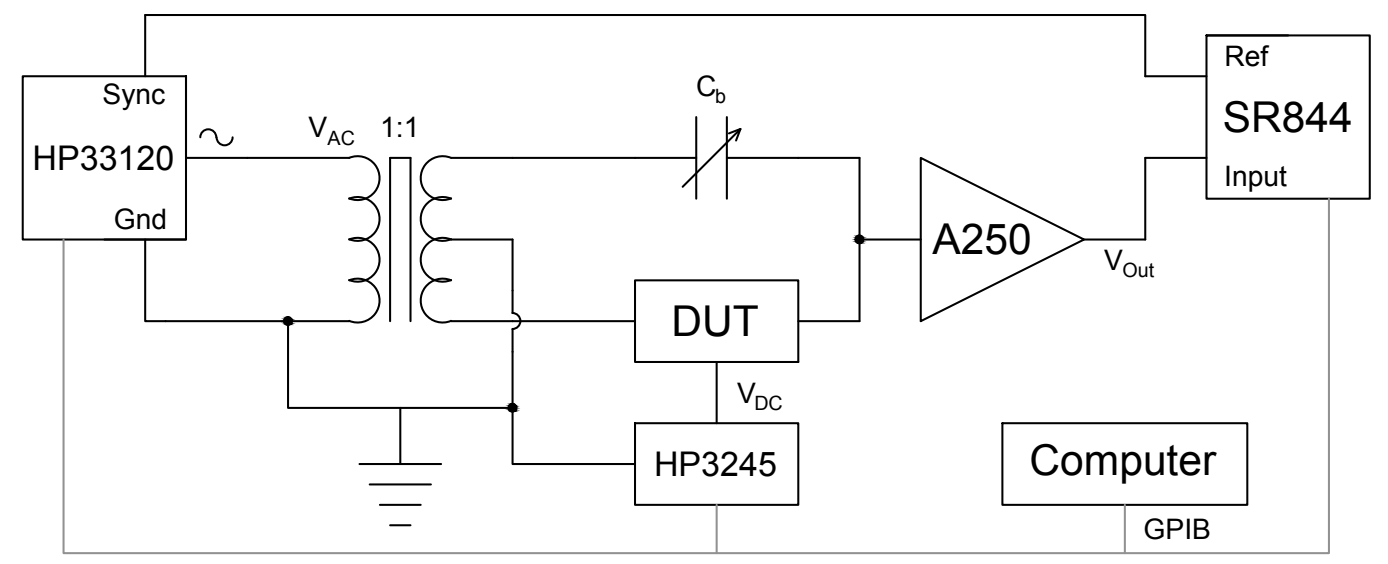

Figure 4.6: Block diagram showing the final experiment setup for electrical measurements.

response at the drive frequency and performs digital to analog conversion [86]. The reference input of the SR844 is fed a square wave from the sync output of the HP 33120A, allowing strong rejection of interfering signals at other frequencies [87].

The remaining spurious response of the system is due to unavoidable capacitive coupling between actuation and sensing electrodes (and their associated bond wires) on the device itself. This leads to an output signal that is the sum of the device response and a static background signal. An attempt to eliminate this was made by balancing out the effects of this parasitic capacitance using a variable capacitor and a phase-shifted copy of the drive signal generated by a center-tapped transformer. The balancing signal is ideally of equal magnitude but $180^{\circ}$ out of phase which results in a cancelation at the input of the amplifier.

In the ideal case, where perfect cancelation is achieved, the entire response of the combined circuit is due to the resonator response. In practice this was not perfectly achieved due to experimental difficulties in precisely adjusting the balancing capacitor. The required value was on the order of $100 \mathrm{fF}$ and getting even $1 \%$ matching requires extremely small capacitance changes. The effect of this imperfect balance is discussed in detail below.

The experiment is controlled by custom software written in National Instruments' LabVIEW programming language [88]. The computer is connected to the instruments via an Agilent 82357A GPIB to USB adaptor. The control software is capable of running automatic frequency sweeps, including multiple sweeps with varying drive and bias voltages.

In order to reduce the damping effects of air on the mechanical structure the electrical experiments were carried out under vacuum. Reducing the damping 
Masters Thesis - Christopher Pope McMaster University - Eng. Physics

coefficient increases the quality factor of the resonator, thereby increasing the amplitude of the response at resonance. This makes the response easier to detect, especially against a noisy background. To enable this the device was placed in a vacuum chamber consisting of a pyrex bell jar on an aluminum base plate. The chamber was evacuated by a Alcatel Drytel 31 dry pump to a pressure of roughly $5 \mathrm{~m}$ Torr. Electrical connections were made by means of coaxial passthrough connectors in the baseplate.

\subsubsection{Experimental challenges}

One experimental setback suffered during electrical testing was the catastrophic failure and destruction of a device. While attempting to improve the signal-to-noise ratio (SNR) of an early device, the bias voltage was increased to approximately $40 \mathrm{~V}$. This caused a catastrophic failure of the oxide under the bridge anchor, shorting it to ground and causing significant damage to the device, as shown in Figure 4.7. It is believed that the failure occurred at one of the alignment markers where the device layer had been etched away, however the exact failure mode is unknown.

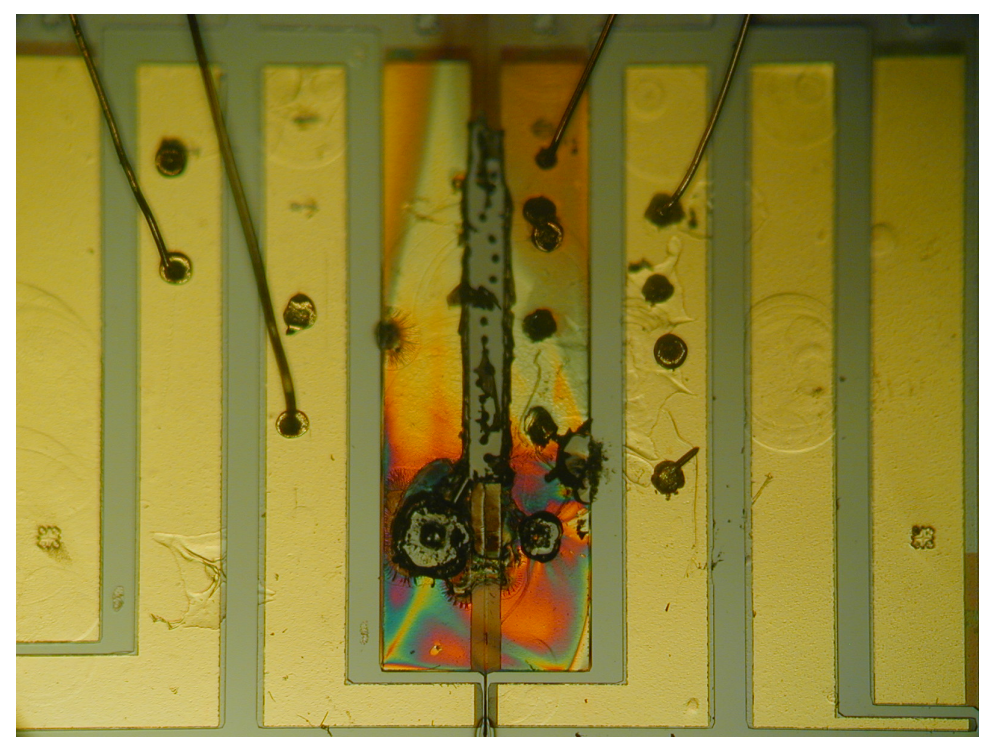

Figure 4.7: Micrograph of the device which suffered catastrophic failure. 


\subsection{Resonance Measurements}

\subsubsection{Results}

The experimental setup shown in Figure 4.6 and described above allows the response of the resonator to the drive signal to be measured as a function of several parameters. The most important parameter is the drive frequency, while the response to changing drive and bias voltages are also of interest. Plotting the response of the resonator as a function of frequency gives a result similar to that shown in Figure 2.8, where the peak amplitude occurs at the resonance frequency $f_{r}$. Figure 4.8 shows the measured response of a resonator with a bridge length of $1000 \mu \mathrm{m}$ in its fundamental in-plane mode, demonstrating a quality factor of 5800. Figure 4.9 shows the response of a $600 \mu \mathrm{m}$ bridge-resonator with a quality factor of 6300 . From plots such as these the resonant frequency and quality factor of the resonators can be determined. Results from such determinations are given in Table 4.3 , along with theoretical resonant frequencies.

The device response curve depends on a number of factors, including the

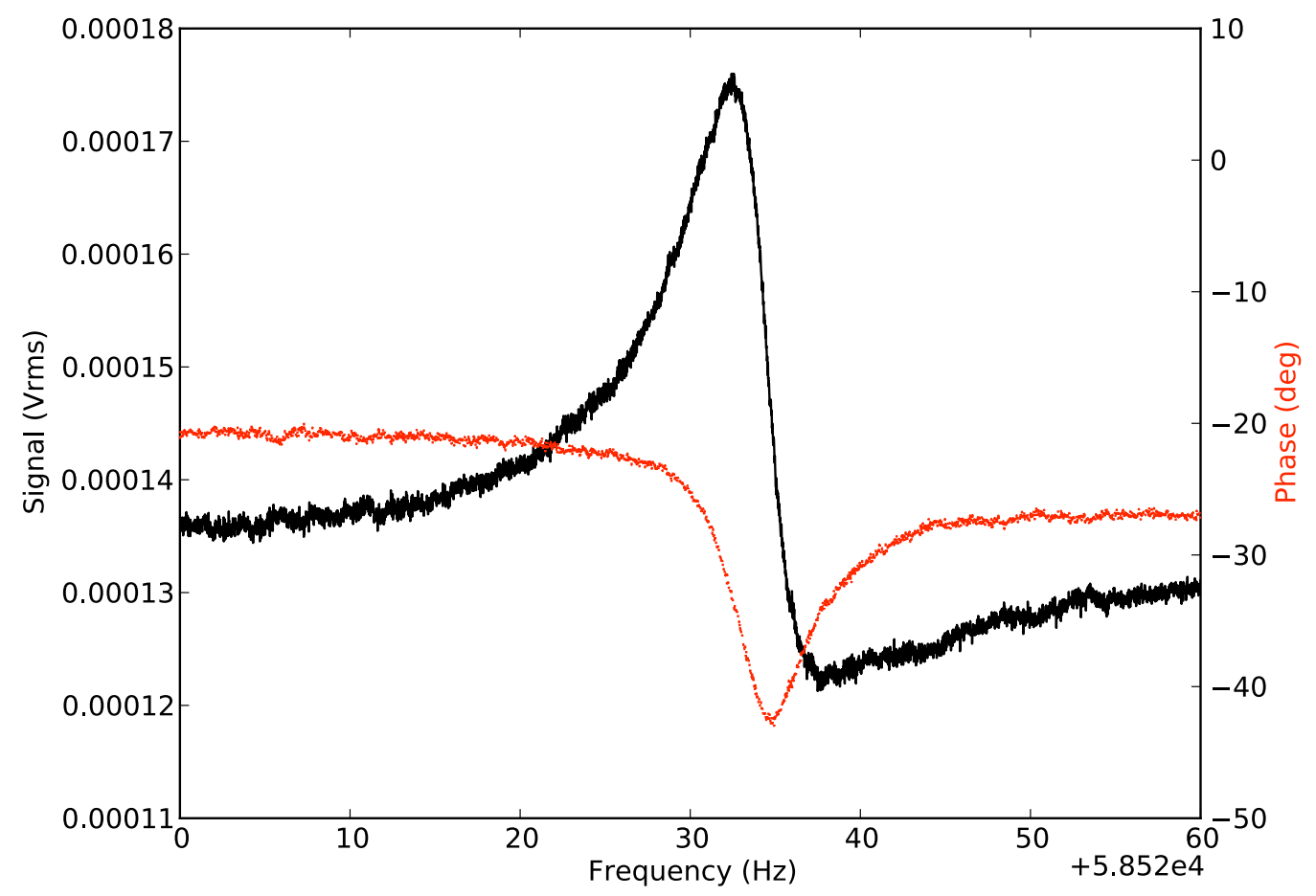

Figure 4.8: Plot showing the response at resonance of a $1000 \mu \mathrm{m}$ bridge resonator in response to a drive voltage of $10 \mathrm{mV}$ and a bias voltage of $5 \mathrm{~V}$. The quality factor of this resonance is approximately 5800 . 
Masters Thesis - Christopher Pope McMaster University - Eng. Physics

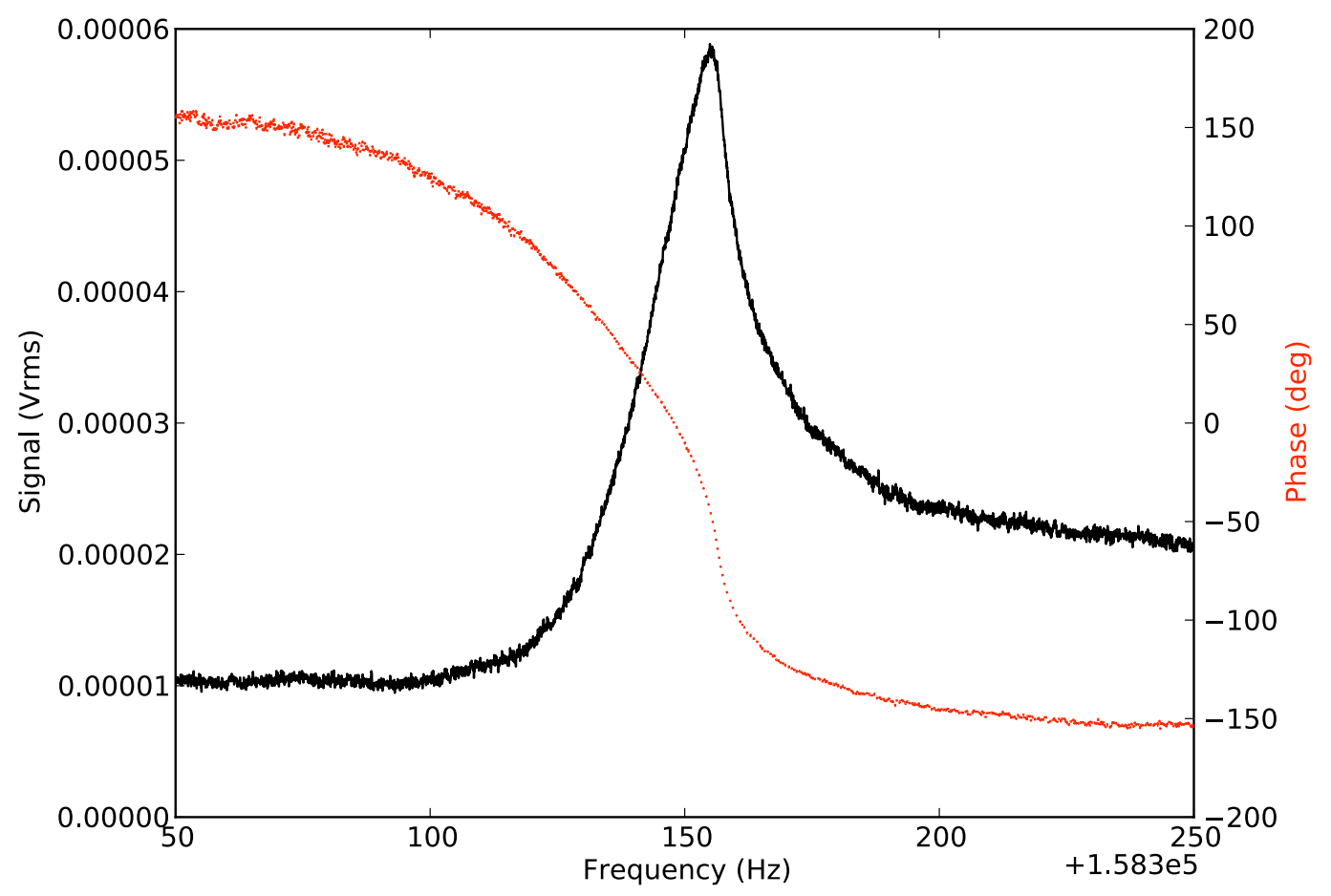

Figure 4.9: Plot showing the response at resonance of a $600 \mu \mathrm{m}$ bridge resonator in response to a drive voltage of $500 \mathrm{mV}$ and a bias voltage of $5 \mathrm{~V}$. The quality factor of this resonance is approximately 6300 .

actuation conditions, the balancing circuit and some apparently random drifts. The stability of a resonance curve with time is illustrated in Figure 4.10 showing the change in curve shape over the course of nearly a day. It may be noted that the curve in Figure 4.8 and the response in general does not perfectly match the theoretical response shown in Figure 2.8. Some of the causes of this are discussed in Section 4.2.2.

A significant divergence exists between the analytically predicted resonant frequencies of the devices and the measured results. This is most likely due to a combination of variations in the manufacturing process and inaccuracies in the approximations used to make the predictions. A narrower than expected bridge would result in a lower than predicted resonant frequency. This may be caused by undercutting of the mask during the anisotropic etching step. The bridge length is greater than the design length due to etching of the anchors, producing the taper shape shown in Figure 3.1. This will also cause a decrease in the resonant frequency. 
Masters Thesis - Christopher Pope $\quad$ McMaster University - Eng. Physics

\begin{tabular}{ccccc}
\hline & & & & \\
Length $(\mu \mathrm{m})$ & $f_{r}(\mathrm{kHz})$ & $Q$ & $f_{a}(\mathrm{kHz})$ & $f_{s}(\mathrm{kHz})$ \\
\hline & & & & \\
200 & - & - & 2229 & \\
400 & - & - & 557 & \\
600 & 158 & 6300 & 248 & \\
800 & - & - & 139 & \\
1000 & 58.5 & 5800 & 89.1 & \\
\hline
\end{tabular}

Table 4.3: Summary of experimental, analytical and simulated resonant frequencies. Experimental frequencies are approximate as they vary with the drive and bias voltages. Frequency is $\pm 2 \%$, quality factor is $\pm 10 \%$ based on observation. Quality factor was determined from Figure 4.8 for the $1000 \mu \mathrm{m}$ device, and from Figure 4.9 for the $600 \mu \mathrm{m}$ bridge. These Figures are representative of the device response. Analytic frequencies are calculated using Equation (2.77) using the Young's modulus for the $\langle 211\rangle$ direction from (2.68).

\subsubsection{Signal and background}

As discussed above, there are parasitic capacitances which inject some fraction of the drive signal into the response of the resonator. This background signal is difficult to prevent or filter out and contributes to some extent to the measured response of the device in all cases. A balancing technique was employed to greatly reduce the contribution of this background signal to the measured response, which improves the sensitivity of the experiment. Figure 4.11 compares the signal measured with and without the addition of the balancing arm.

This background signal can potentially distort both the measured amplitude and the phase of the device response. The input signal, and thus the device response, is measured by the lock-in amplifier in terms the in-phase component $X$ and the out-of phase component $Y$ The signal components are measured at a $90^{\circ}$ phase shift, with the in-phase component being in phase with the reference signal. When the resonator response is combined with the background signal, these components may be expressed as

$$
X=X(\omega)+X_{b} \quad Y=Y(\omega)+Y_{b}
$$

which again take the form of a frequency-dependant response against a static background.

\footnotetext{
${ }^{4}$ These components are sometimes labeled $I$ and $Q$ (for quadrature)
} 
Masters Thesis - Christopher Pope McMaster University - Eng. Physics

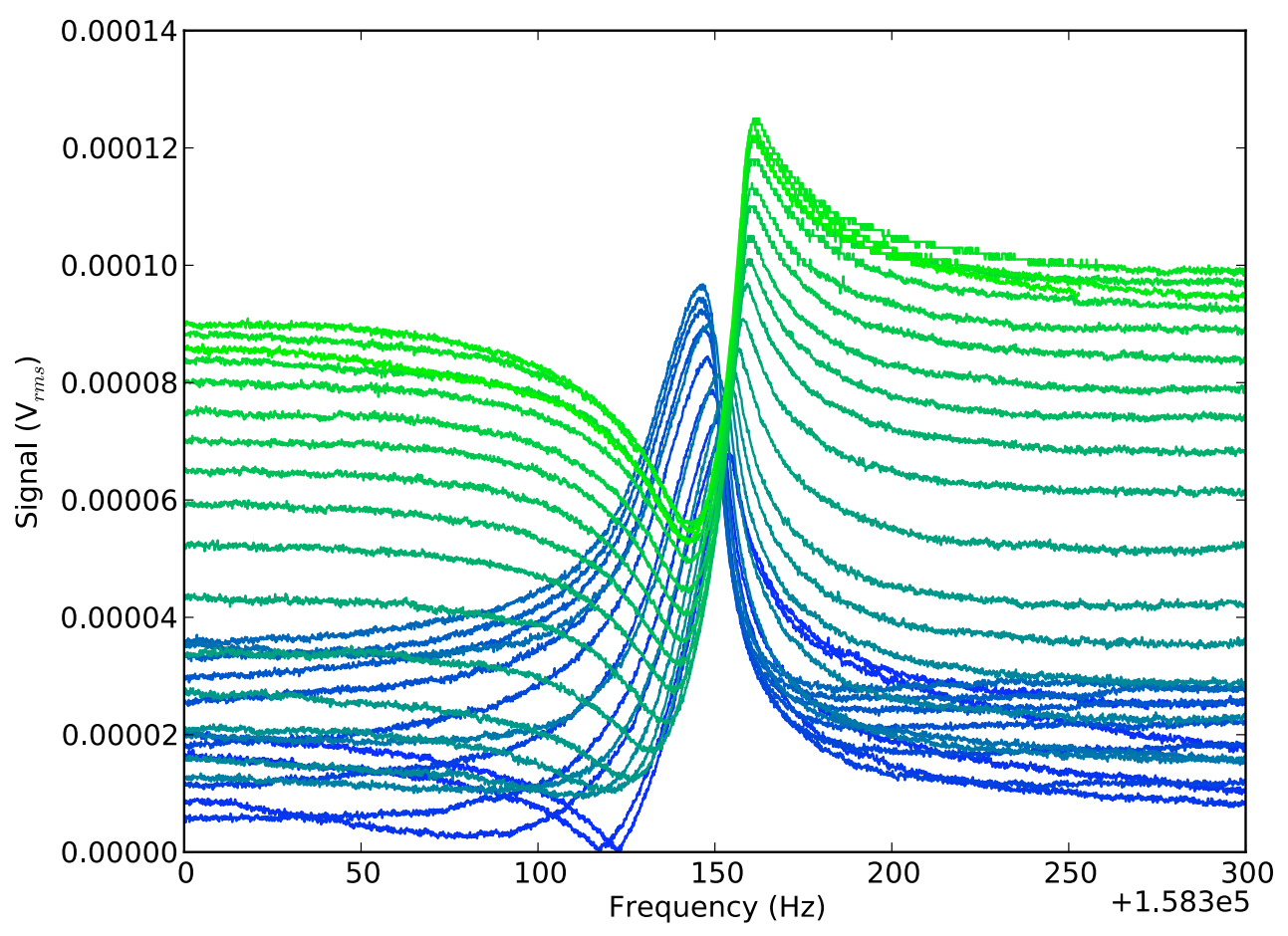

Figure 4.10: Plot showing stability of the resonance peak over time. The first sweep is plotted in green, the last sweep is plotted in blue and the intervening sweeps show a gradient. The data set contains 28 sweeps taken over 21 hours, each sweep lasting 45 minutes. Changes in the shape and position of the curves may be due to drifts in the device response, the balancing circuit or the measurement equipment. Actuation is at $500 \mathrm{mV}$ with a $5 \mathrm{~V}$ bias.

The quantities of interest for analyzing the resonator response are the magnitude

$$
R=\sqrt{X^{2}+Y^{2}}
$$

and the phase

$$
\phi=\arctan \frac{Y}{X}
$$

which correspond to the analysis in Section 2.5.2. If the background signal is large relative to the resonator response then the magnitude of the change in the calculated phase will be significantly smaller than the change in the phase of the real resonator response. This effect was observed experimentally. The effect of the background on the calculated magnitude is more complex, and this is believed to explain the difference between the shape of the experimental resonance curves and the theoretical response of a simple harmonic oscillator. 
Masters Thesis - Christopher Pope McMaster University - Eng. Physics

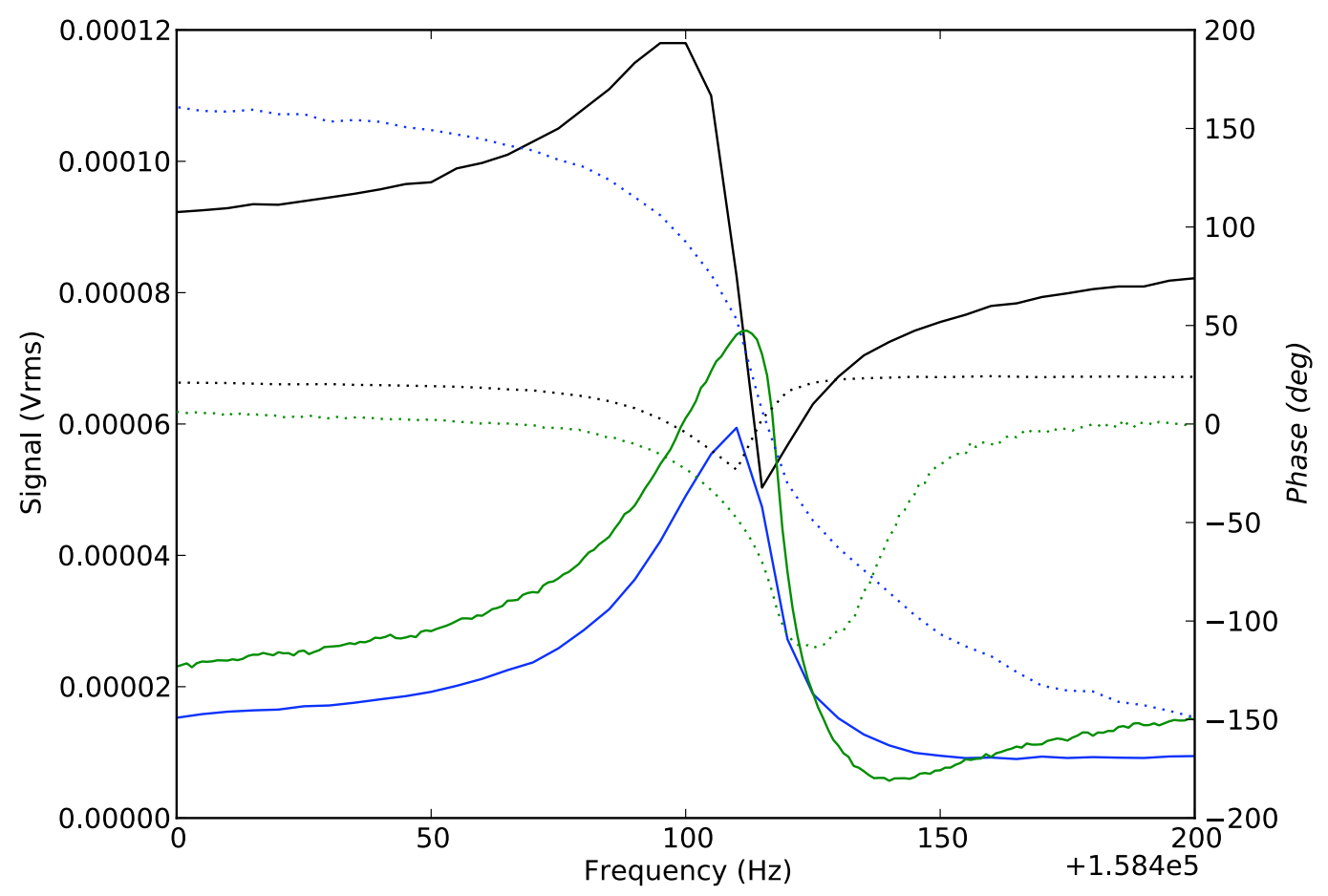

Figure 4.11: Plot showing the device response improved balancing. The background signal is reduced (shifted down) and the phase change increases.

\subsubsection{Overdriving}

The phenomenon of pull-in, whereby a sufficient bias voltage across the resonatorelectrode gap might cause collapse of the structure was discussed in Section 2.5.5. That analysis was performed at DC and does not take into account the quality factor of the resonator which increases its motion at resonance, potentially by several orders of magnitude. Measurements of resonators made at high bias voltages and, in general high values of the average drive voltage $V_{\text {avg }}=\sqrt{V_{A C} V_{D C}}$ lead to the observation of a clipping behavior in the device response. A characteristic plot of this clipped response is shown in Figure 4.12 .

This clipped response takes the form of a sharp drop in the signal amplitude as the drive frequency approaches the resonant frequency of the structure. Many such drops and recoveries are observed as the drive passes through resonance until the device settles to its lower response above resonance. Close examination of this effect revealed that this clipping behavior happened between adjacent data points even as the frequency step between each point was reduced significantly. The cause of this clipping behavior appears to be an excessive amplitude of the actuation force resulting from the product of the 


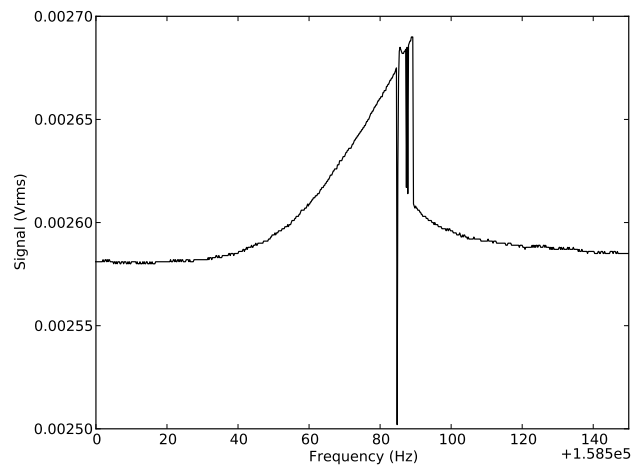

(a) $1 \mathrm{~V}$ actuation

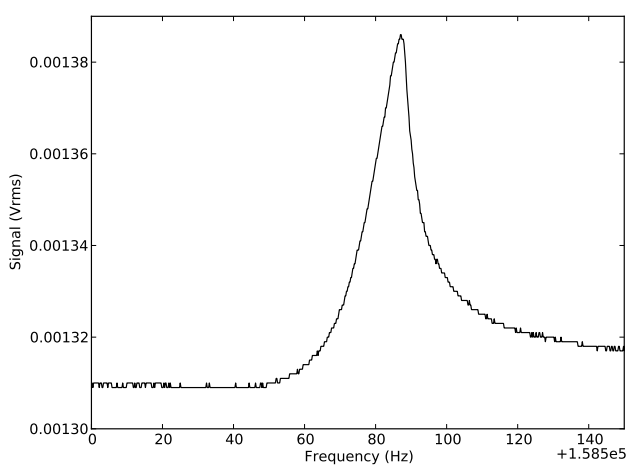

(b) $0.5 \mathrm{~V}$ actuation

Figure 4.12: Contrast between overdriven and normal response. Measurements were made consecutively on a $600 \mu \mathrm{m}$ bridge under $5 \mathrm{~V}$ bias.

actuation and bias potentials exceeding some limit. Reducing the actuation amplitude reduced and then eliminated this distortion.

While the physical mechanism behind the clipping response has not been proven, a reasonable theory may be developed. Recalling from (2.134) that the current response of the resonator is proportional to its velocity, the sudden drop in output is likely due to a sharp drop in that velocity. The proposed cause for this sudden drop in velocity is the resonator coming into contact with an electrode due to the greatly increased amplitude of its motion at resonance $5^{5}$ This drop in velocity would persist for a time period

$$
\tau=2 \pi Q / \omega_{r}
$$

called the ring-down time, where $Q$ is the quality factor. This period is less than one second for the devices measured here which would explain the intermittent nature of the effect. It should be noted that while the cause of this effect has not been proven experimentally the relationship to the actuation voltage has been demonstrated. This is sufficient to allow the effect to be avoided, as has been done in subsequent experiments.

\subsection{Effective Spring Constant}

In Section 2.5.5 it was determined that a symmetric electrostatic force acting on a MEMS device could cause a reduction in the effective stiffness of the device. The electrode geometry described here allows such a force to be applied,

\footnotetext{
${ }^{5}$ It may be considered surprising that the resonator survives such repeated collisions and is a testament to the durability of MEMS devices.
} 
which was accomplished by applying a DC bias voltage to the bridge, placing a potential drop across the gaps formed between the bridge and grounded electrodes. This was accomplished by grounding the unused electrodes (through $50 \Omega$ SMA terminators). Additionally, the amplifier and signal generator are virtual grounds at DC, meaning the potential is symmetric across all four gaps. The only asymmetry comes from the second term of Equation (2.121) due to the $\mathrm{AC}$ actuation voltage.

This softening of the structure should be visible as a decrease in the resonant frequency of the bridge-resonator with increasing bias voltage. This is exactly what is observed, as shown in Figure 4.13 which combines the results of several frequency sweeps on a $600 \mu \mathrm{m}$ bridge. Figure 4.14 shows this decrease in resonant frequency for bias voltages between $10 \mathrm{~V}$ and $40 \mathrm{~V}$. This causes the resonant frequency of the bridge to decrease by $1.5 \mathrm{kHz}$, or nearly $1 \%$. The decrease is quadratic, as predicted by the stiffness in Equation (2.130). Figure 4.15 shows the increase in response amplitude (normalized to the drive amplitude) with increasing bias, as predicted by Equation (2.137).

A similar effect is observed for a $1000 \mu \mathrm{m}$ device, as shown in Figure 4.16. The more limited bias range of $1 \mathrm{~V}$ to $5 \mathrm{~V}$ is due to experimental limitations

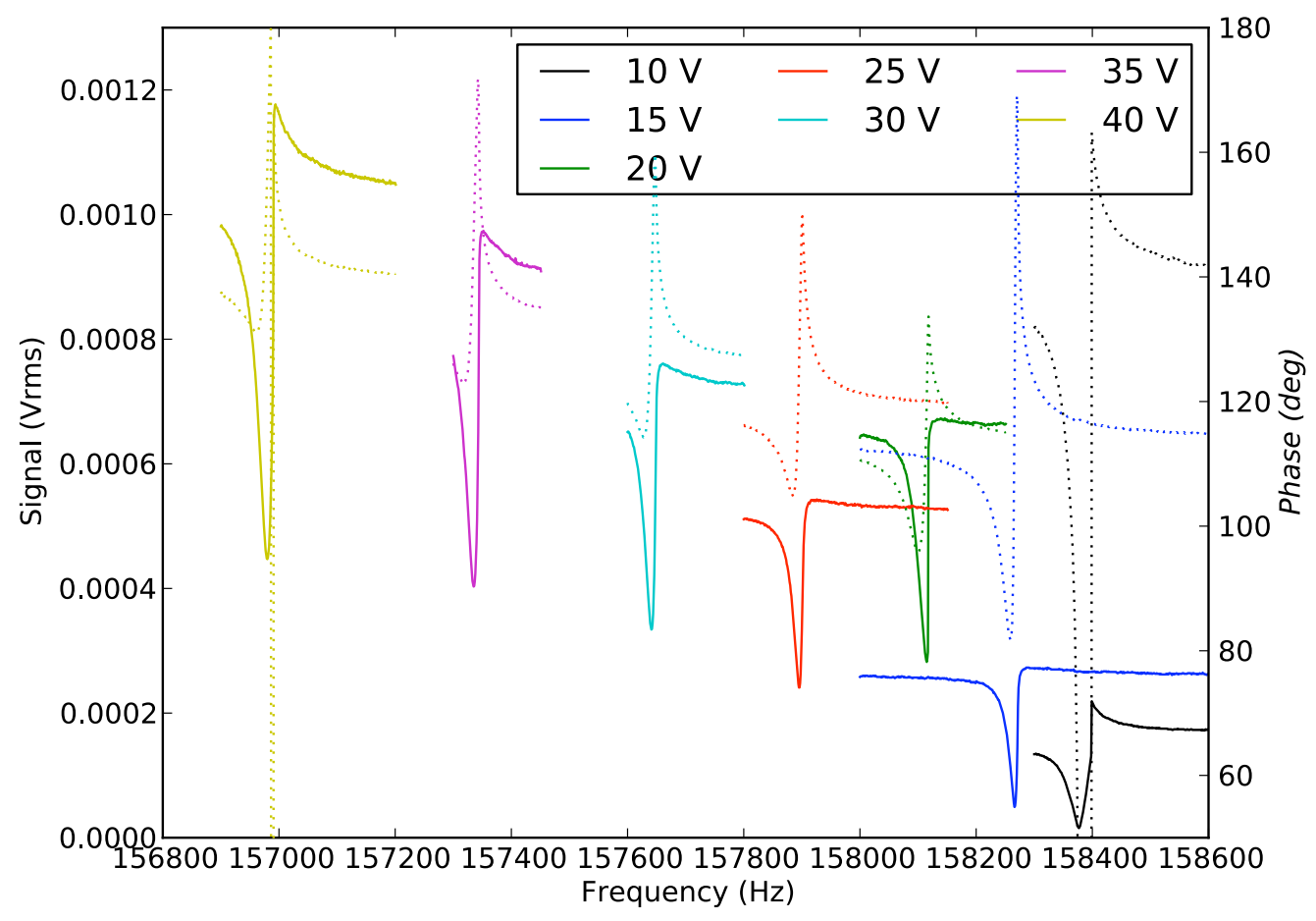

Figure 4.13: Plot showing the shift in resonance frequency and response amplitude of a $600 \mu \mathrm{m}$ resonator due to increasing bias voltage. The drive amplitude varies between $500 \mathrm{mV}$ and $100 \mathrm{mV}$ with increasing bias to prevent clipping. 
Masters Thesis - Christopher Pope McMaster University - Eng. Physics

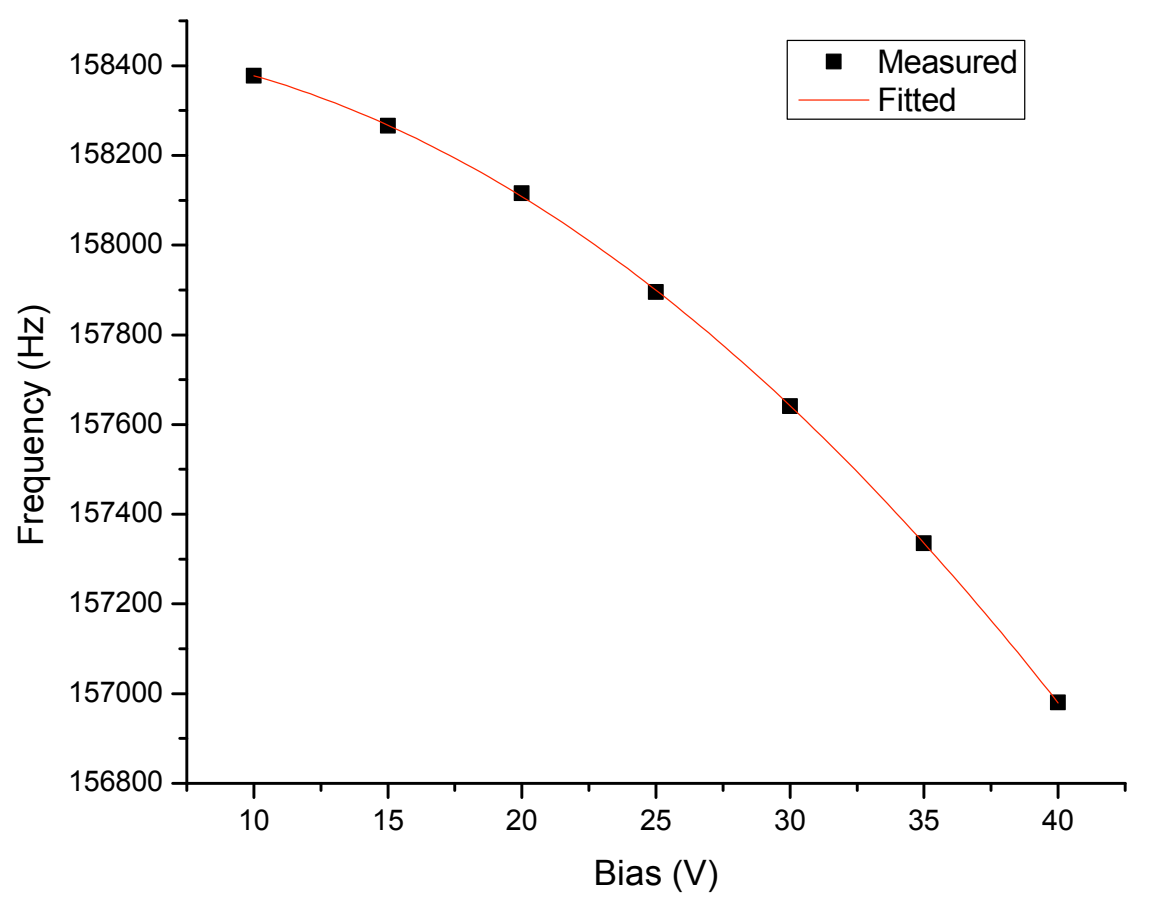

Figure 4.14: Plot of the shift in resonance frequency vs. bias voltage for a $600 \mu \mathrm{m}$ resonator, using the data from Figure 4.16. The polynomial fit shows an intercept at $158451 \mathrm{~Hz} \pm 11 \mathrm{~Hz}$ with a slope of $2.50 \mathrm{~Hz} / \mathrm{V} \pm 0.94 \mathrm{~Hz} / \mathrm{V}$ and a quadratic coefficient of $-0.982 \mathrm{~Hz} / \mathrm{V}^{2} \pm 0.019 \mathrm{~Hz} / \mathrm{V}^{2} .\left(R^{2}=1.00\right)$

involving the vertical pull-in voltage of the structure. Figure 4.17 shows the decreasing frequency as a result of the electrostatic softening effect in the same way as the $1000 \mu \mathrm{m}$ bridge. Figure 4.18 shows the corresponding increase in response amplitude.

The softness of the out-of-plane mode is a limitation when attempting to increase the bias voltage to improve the device response. This is also a key complication when attempting to reduce the effective spring constant of the resonator. The structure is thinner in this direction by roughly a factor of two which gives a factor of four in the stiffness. This in turn means that the pull-in voltage in the vertical direction is less than that for the in-plane motion. Thus the DC bias applied to the bridge is limited by this value, when the substrate is grounded ${ }^{6}$

There are essentially two solutions to this problem. The first is to bias the actuation and sensing electrodes separately, as in Figure 4.19. On the sensing side, the resistor $R_{b}$ must large compared to the input impedance of

\footnotetext{
${ }^{6}$ In practice the bias voltage is limited to be less than the calculated pull-in voltage plus some safety margin as determining the pull-in voltage experimentally risks destroying the device.
} 
Masters Thesis - Christopher Pope McMaster University - Eng. Physics

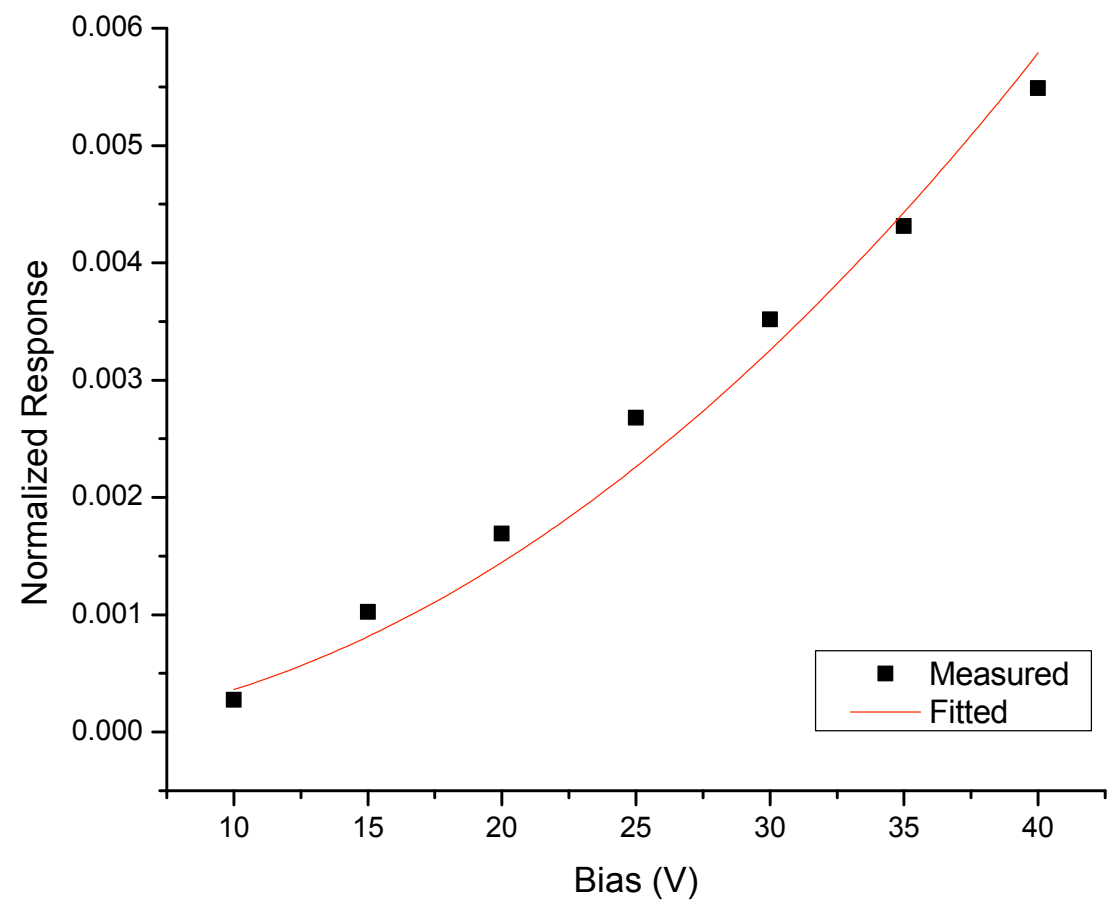

Figure 4.15: Plot of the device response (peak height) vs. bias voltage for a $600 \mu \mathrm{m}$ resonator, using the data from Figure 4.13. The device response has been normalized to the drive amplitude. The curve is quadratic coefficient $(3.62 \pm 0.12) \times 10^{-6} \mathrm{~V} / \mathrm{V}^{2} \cdot\left(R^{2}=0.978\right)$

the amplifier (which is hopefully small) to prevent any of the signal current from flowing into the bias supply instead. The blocking capacitors $C_{b}$ are present to protect the drive and detect electronics from the $\mathrm{DC}$ voltages. This approach has the benefit of allowing independent control over the bias voltages. This is in fact a disadvantage when trying to match the bias voltages to soften the bridge as precise matching of the bias supplies may be required.

A simple alternative available in this geometry is to simply tie the bridge and substrate together, placing them at the same potential. This provides all the benefits of biasing the bridge while eliminating the electrostatic force between the bridge and substrate. The noise performance should be similar as the DC supply ideally offers a low AC impedance to ground. Isolating the substrate was accomplished by placing an isolated pad on the PCB to accept the chip and providing it with an independent electrical connection, as seen in Figure 4.2. A solder jumper was provided to easily ground the substrate if required. Future PCB revisions may tie the bridge and substrate together directly. This technique will be useful for future optical experiments which intend to induce small changes in the stiffness of the structure. Reducing the 
Masters Thesis - Christopher Pope McMaster University - Eng. Physics

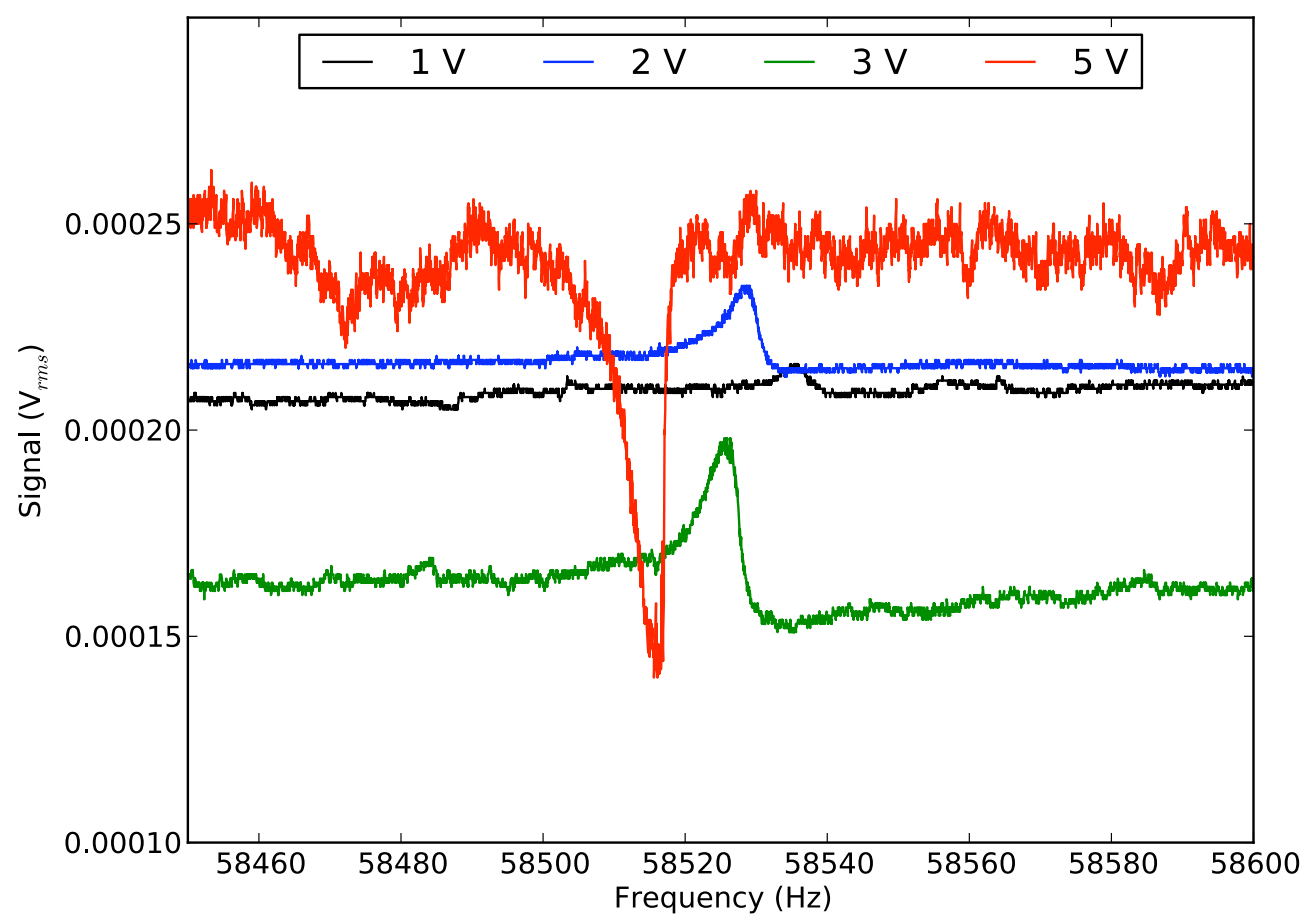

Figure 4.16: Plot showing the shift in resonance frequency and response amplitude of a $1 \mathrm{~mm}$ resonator due to increasing bias voltage. Actuation is at $200 \mathrm{mV}$.

stiffness electrostatically will allow small absolute changes in the stiffness to cause larger relative changes. 
Masters Thesis - Christopher Pope McMaster University - Eng. Physics

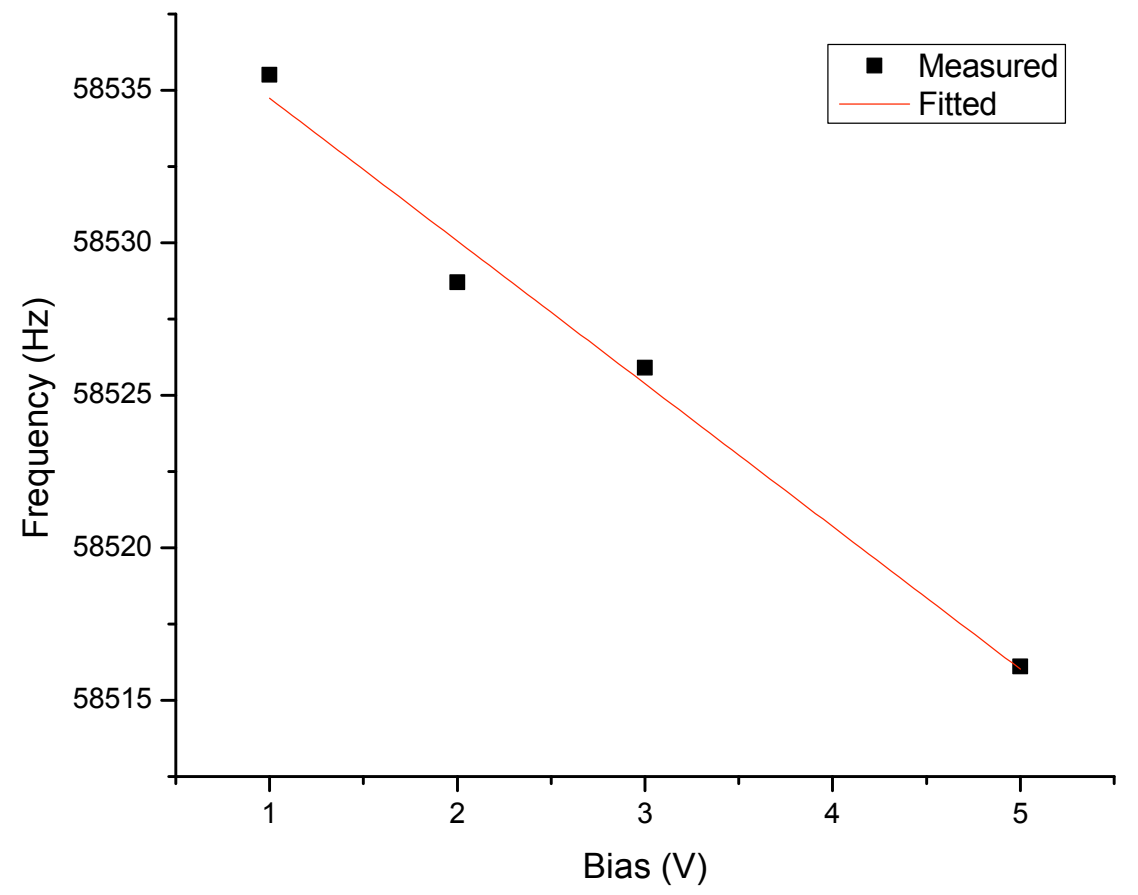

Figure 4.17: Plot of the shift in resonance frequency vs. bias voltage for a $1 \mathrm{~mm}$ resonator, using the data from Figure 4.16. The linear fit shows an intercept at $58539.4 \mathrm{~Hz} \pm 1.2 \mathrm{~Hz}$ with a slope of $-4.68 \mathrm{~Hz} / \mathrm{V} \pm 0.39 \mathrm{~Hz} / \mathrm{V} .\left(R^{2}=0.979\right)$ 
Masters Thesis - Christopher Pope McMaster University - Eng. Physics

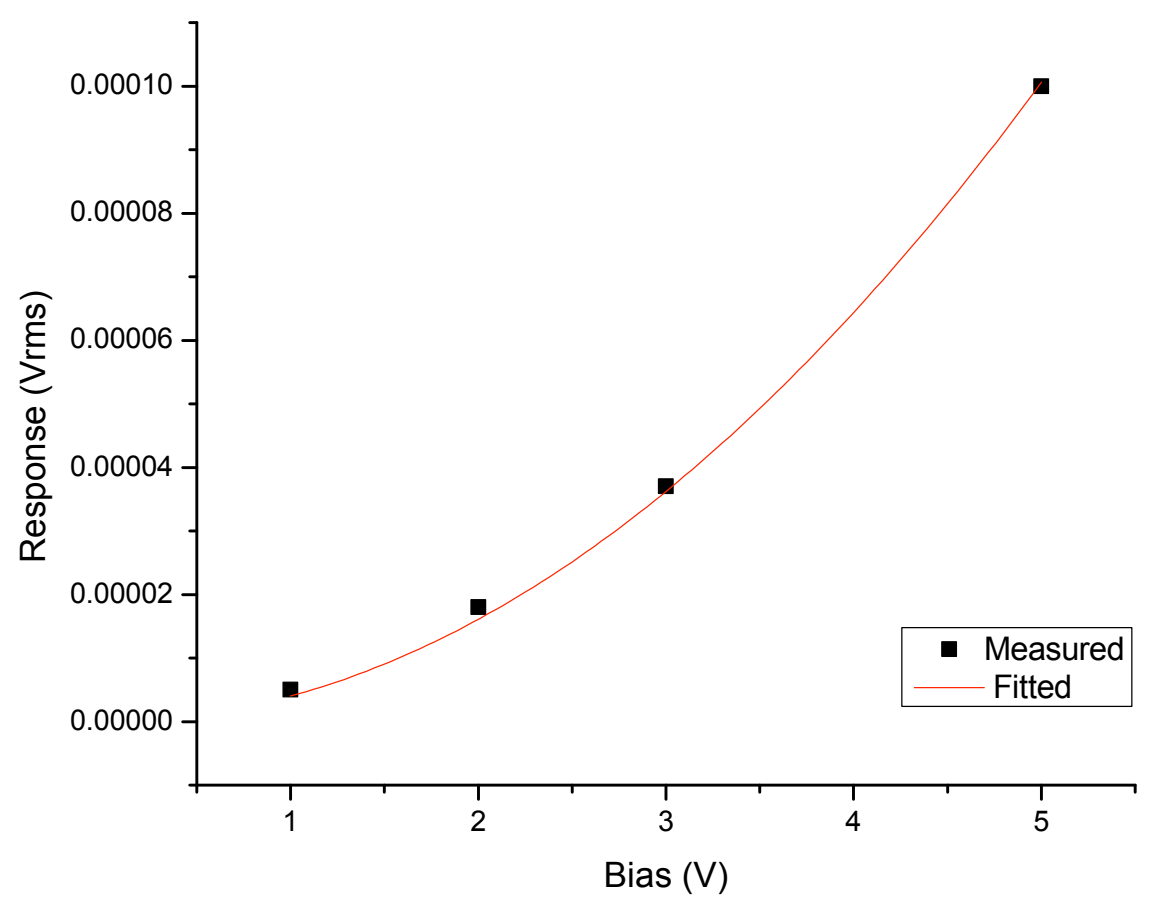

Figure 4.18: Plot of the device response (peak height) vs. bias voltage for a $1 \mathrm{~mm}$ resonator, using the data from Figure 4.16. The curve is quadratic coefficient $(4.024 \pm 0.051) \times 10^{-6} \mathrm{~V} / \mathrm{V}^{2} .\left(R^{2}=0.999\right)$

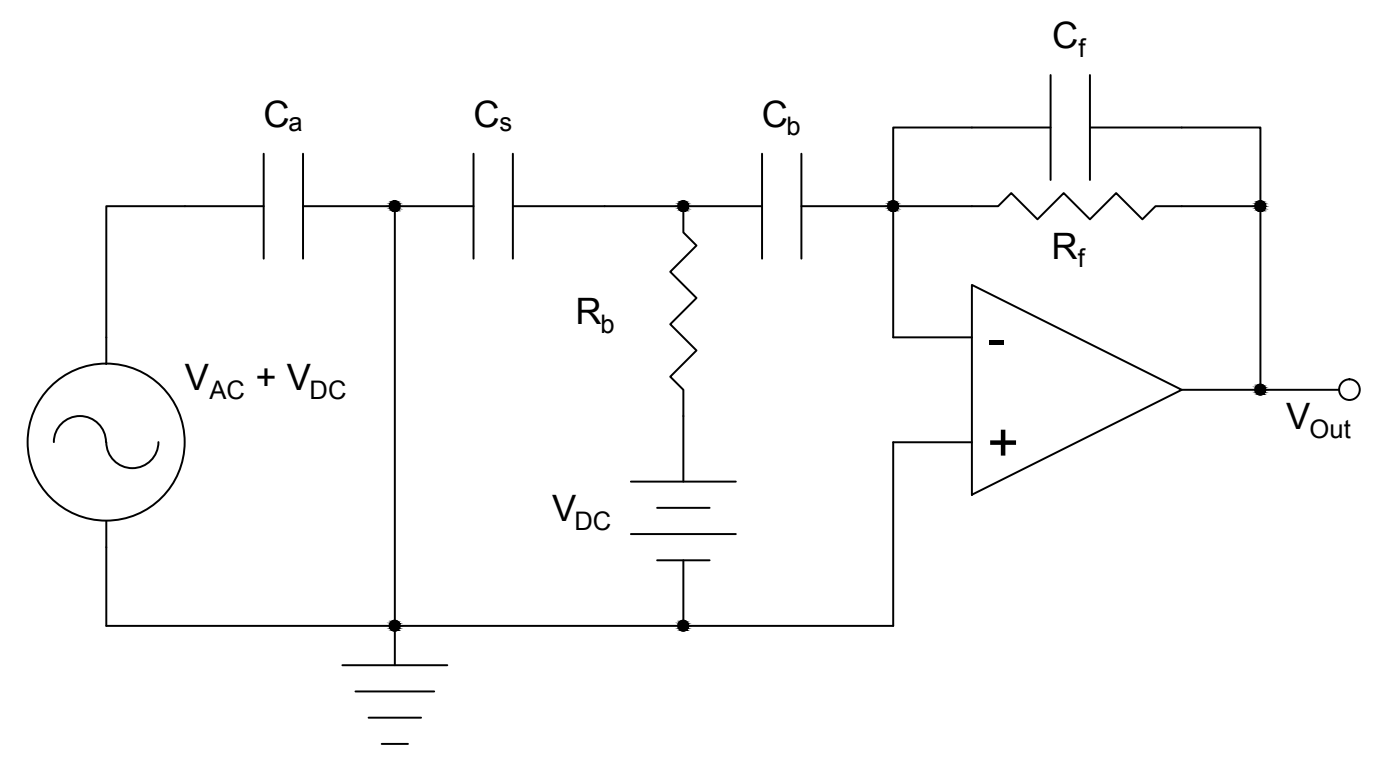

Figure 4.19: Schematic showing the circuit for separately biasing the actuation and sensing electrodes. 


\subsection{Optical Experiment}

The electrical and mechanical device design described here is planned to allow two key optical experiments to be performed. The first is to measure the effective stiffness of the optical power confined in the waveguide, as described in Section 2.2.3. This effect may be observed as a shift in the resonance frequency of the structure due to a change in the total stiffness of the structure with increasing optical intensity. Such an effect does not appear to have been previously proposed in the literature. The ability to reduce the effective spring constant of the structure, as demonstrated in Section 4.3 allows an optical stiffness to be observed more readily by increasing the relative magnitude of the optical stiffness vs. the mechanical stiffness.

The second experiment would be to actuate the device optically and observe the resulting resonance capacitively, as has been done for electrical actuation. In order to apply an optical force to the structure, the waveguide must be pre-curved, as the force is proportional to the curvature. This curvature can be applied electrostatically by means of a DC bias between the bridge and an electrode, which would induce a deflection of the bridge. If the light coupled into the waveguide is pulsed at a frequency $f$ then the structure will experience a force opposing the curvature that is pulsed at the same frequency. Such a force can be used to excite the structure at resonance.

The devices described above were designed and fabricated for the purpose of demonstrating this effect. Introducing light into the waveguides may be accomplished by means of butt coupling from the face of a cleaved optical fiber to the end-face of the waveguide. This requires careful alignment of the $10 \mu \mathrm{m}$ fiber core to the $4 \mu \mathrm{m}$ waveguide cross-section to provide maximum coupling. To allow this coupling, the system shown in Figure 4.20 was constructed using a combination of commercial optical components and custom mounting brackets.

Using this setup, coupling of light into the waveguides was attempted. The light source was a $1550 \mathrm{~nm}$ semiconductor laser (JDS Fitel custom assembly) coupled via an FC connector to a single-mode fiber.7 Despite best efforts, coupling of light into the waveguides could not be demonstrated. This is believed to be due to the low quality of the waveguide facets. Efforts to fabricate devices with improved waveguide and facet quality are underway.

\footnotetext{
${ }^{7}$ A standard telecom fiber, Corning SMF-28 was used. For better coupling efficiency a tapered fiber was on hand, but not used. Both fibers were purchased from OZ Optics of Ottawa, Ontario.
} 


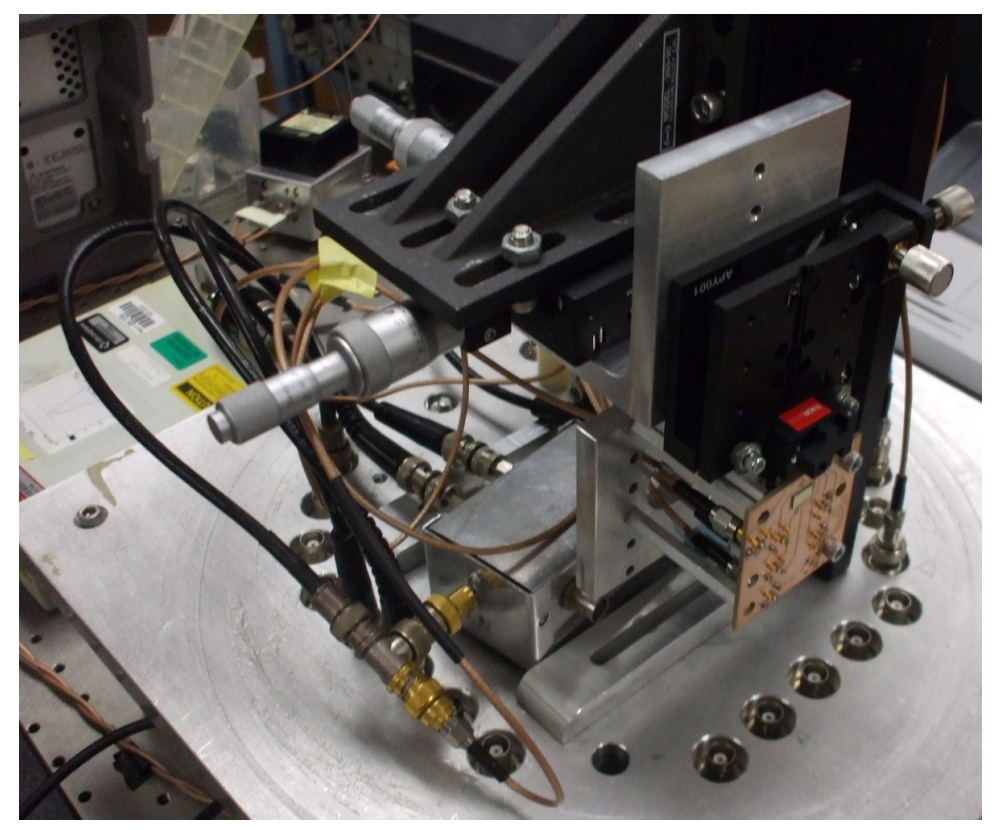

Figure 4.20: Photograph of the assembled electrical-optical-mechanical experiment. The system is mounted to a baseplate which accepts a bell jar, allowing the experiment to be carried out under vacuum. 


\section{Chapter 5}

\section{Conclusion}

\subsection{Summary}

This thesis describes the design, fabrication and operation of silicon micromechanical resonators and a procedure for using these devices to measure radiation pressure due to light confined in a waveguide. The resonators detailed here consist of a fixed-fixed beam fabricated and released from a SOI wafer by wet chemical etching. A process flow for this fabrication was developed, photolithographic masks were designed and the process flow was executed. These bridges were actuated and their motion sensed by capacitive methods. The frequency response of the resonators was used to locate and excite the fundamental in-plane mode of the bridge. Electrostatic tuning of the resonance frequency via the effective stiffness of the structure was described theoretically and demonstrated experimentally.

A number of challenges have been identified in the fabrication process, in the electrical experiment and in the optical experiment. Causes and potential solutions have been discussed, with many solutions being demonstrated. The usefulness of printed circuit boards as low-cost custom test jigs has been demonstrated, with results which improve on other available options. Additionally, the techniques necessary for detecting resonant behavior against background signals have been developed and implemented.

The optical experiment developed in this thesis represents a novel technique for the measurement of electromagnetic momentum. The MEMS devices needed to perform this experiment have been designed, fabricated and

tested. The electrical, mechanical and software support infrastructure has been designed, acquired, and integrated as necessary. 


\subsection{Future Work}

\subsubsection{Optical experiment}

This thesis has described an experiment which would allow the measurement of the optical momentum carried by light in a waveguide. This measurement was to be performed by the effect of radiation pressure acting inside a curved waveguide which was also a mechanical resonator. This geometry does not appear to have been previously proposed for measuring radiation pressure. A successful implementation of this experiment with sufficient resolution would allow a statement to be made regarding the Abraham-Minkowski controversy regarding the correct magnitude of the optical momentum in a medium.

Making a quantitative measurement of the momentum carried by light in the waveguide would represent a significant scientific contribution. As the proposed experiment is different from those carried out so far it would provide a unique perspective on the Abraham-Minkowski controversy. An argument can be advanced for the Abraham form of the photon momentum being the obvious result. This is due to the optical force acting inside the mechanical structure and its interaction with the motion of that structure. This would fit with the identification of the Abraham momentum as the mechanical momentum carried by light. An appropriate formulation of the problem should also allow the role of the Minkowski momentum to be demonstrated, as the light is indeed propagating inside the medium. This would be a demonstration of both forms simultaneously and provide experimental evidence for their equivalence, as has been previously discussed.

While the fabrication and characterization of the mechanical system has been completed, unforeseen experimental difficulties have delayed the optical experiment. As the infrastructure for this experiment is in place it should be relatively straight-forward to complete this measurement in the future. Existing designs may be used for the device and the PCB mount. The only remaining fabrication challenge is the preparation of optical quality waveguide facets. Coupling light into the waveguides is the only remaining experimental challenge, aside from whatever work may be needed to increase the sensitivity of the experiment.

\subsubsection{Revised device design}

A new revision of the mask design would allow several improvements to be made which have the potential to make the experiment easier and more sensitive. The first is to locate electrical contacts farther away from the waveguide facet, separating the electrical and optical connections to the device. Additionally, it would be beneficial to design a structure which implements the balancing circuit on the same chip as the device. This would allow the reduc- 
tion of the background signal by nulling it though a structure matched to the resonator.

The sensitivity of the future optical experiment could be improved by two main changes. Since the experiment seeks to measure small changes in the stiffness of the mechanical structure, it is desirable to decrease the initial stiffness so that the relative magnitude of the change is maximized. One way to decrease the stiffness of the bridge is to increase its length, as the stiffness is proportional to $1 / L^{3}$. This work has demonstrated the feasibility of releasing $1 \mathrm{~mm}$ bridges. However that does not appear to be a maximum and longer structures may be possible. A second option is to fabricate cantilevers instead of bridges, as the stiffness is lower.

A third technique for decreasing the stiffness of the fabricated devices would be to decrease the bridge width, as the stiffness is proportional to $w^{3}$ for the in-plane mode. The current fabrication techniques are near their limit with respect to alignment of the waveguide on the bridge, however these are not fundamental physical limitations. Taking advantage of improved optical lithography processes or electron beam lithography would allow the width and therefore the stiffness of the structures to be reduced. The lower limit on the bridge stiffness would then be due to the need to release the structure.

\subsubsection{Electrostatic softening}

The results described in Section 4.3 may be considered preliminary work in this area. The symmetry of the bridge design allows, in theory, for the effective stiffness of the structure to be decreased to zero. An expanded demonstration of this effect would be useful for the tuning of MEMS resonators, which would have applications for both frequency references and RF filters. Carrying out this experiment should be straightforward, taking advantage of the techniques demonstrated in this thesis. 


\section{References}

[1] J. Maxwell, A treatise on electricity and magnetism., 1st ed. Oxford, 1873. [Online]. Available: http://posner.library.cmu.edu/Posner/books/ book.cgi?call=537_M46T_1873_VOL._2

[2] J. Maxwell, A treatise on electricity and magnetism., unabridged and unaltered republ. of 3. ed. 1891. ed. New York: Dover Publ., 1954.

[3] E. F. Nichols and G. F. Hull, "A preliminary communication on the pressure of heat and light radiation," Physical Review (Series I), vol. 13, no. 5, p. 307, Nov. 1901. [Online]. Available: http://link.aps.org/doi/10.1103/PhysRevSeriesI.13.307

[4] E. F. Nichols and G. F. Hull, "The pressure due to radiation," Proceedings of the American Academy of Arts and Sciences, vol. 38, no. 20, pp. 559-599, Apr. 1903. [Online]. Available: http://www.jstor. org/stable/20021808

[5] P. Lebedew, "Untersuchungen über die druckkräfte des lichtes," Annalen der Physik, vol. 311, no. 11, pp. 433-458, Jan. 1901. [Online]. Available: http://onlinelibrary.wiley.com/doi/10.1002/andp.19013111102/abstract

[6] A. Ashkin, "Acceleration and trapping of particles by radiation pressure," Physical Review Letters, vol. 24, no. 4, p. 156, Jan. 1970. [Online]. Available: http://link.aps.org/doi/10.1103/PhysRevLett.24.156

[7] A. Ashkin, "Optical levitation by radiation pressure," Applied Physics Letters, vol. 19, p. 283, 1971. [Online]. Available: http: //link.aip.org/link/?APL/19/283/1

[8] A. Ashkin, J. M. Dziedzic, J. E. Bjorkholm, and S. Chu, "Observation of a single-beam gradient force optical trap for dielectric particles," Optics Letters, vol. 11, no. 5, pp. 288-290, May 1986. [Online]. Available: http://ol.osa.org/abstract.cfm?URI=ol-11-5-288

[9] A. Ashkin and J. Dziedzic, "Optical trapping and manipulation of viruses and bacteria," Science, vol. 235, no. 4795, pp. $1517-1520$, 
Masters Thesis - Christopher Pope McMaster University - Eng. Physics

Mar. 1987. [Online]. Available: http://www.sciencemag.org/content/ 235/4795/1517.abstract

[10] S. Chu, L. Hollberg, J. E. Bjorkholm, A. Cable, and A. Ashkin, "Three-dimensional viscous confinement and cooling of atoms by resonance radiation pressure," Physical Review Letters, vol. 55, no. 1, p. 48, Jul. 1985. [Online]. Available: http://link.aps.org/doi/10.1103/ PhysRevLett.55.48

[11] S. Chu, J. E. Bjorkholm, A. Ashkin, and A. Cable, "Experimental observation of optically trapped atoms," Physical Review Letters, vol. 57, no. 3, p. 314, Jul. 1986. [Online]. Available: http: //link.aps.org/doi/10.1103/PhysRevLett.57.314

[12] A. Ashkin, "Optical trapping and manipulation of neutral particles usinglasers," Proceedings of the National Academy of Sciences of the United States of America, vol. 94, no. 10, pp. 4853-4860, May 1997. [Online]. Available: http://www.pnas.org/content/94/10/4853.abstract

[13] W. A. Hollerman, "The physics of solar sails," Apr. 2003. [Online]. Available: http://ntrs.nasa.gov/search.jsp?print =yes\&R=20030093608

[14] "IKAROS ProjectJAXA space exploration center," http://www.jspec.jaxa.jp/e/activity/ikaros.html. [Online]. Available: http://www.jspec.jaxa.jp/e/activity/ikaros.html

[15] "NASA - NanoSail-D home page," http://www.nasa.gov/mission_pages/smallsats/nanosaild.html, NanoSail-D index page. [Online]. Available: http: //www.nasa.gov/mission_pages/smallsats/nanosaild.html

[16] A. Clarke, "The wind from the sun," in The collected stories of Arthur C. Clarke., 1st ed. New York: Tom Doherty Associates, 2001, pp. 828-842.

[17] H. Minkowski, "Die grundgleichungen für die elektromagnetischen vorgänge in bewegten körpern," Nachrichten von der Gesellschaft der Wissenschaften zu Göttingen, Mathematisch-Physikalische Klasse, vol. 53, pp. 53-111, 1908.

[18] H. Minkowski, "Die grundgleichungen für die elektromagnetischen vorgänge in bewegten körpern," Mathematische Annalen, vol. 68, pp. 472-525, Dec. 1910. [Online]. Available: http://www.springerlink.com/ content/x709h877x251881u/ 
[19] M. Abraham, "Zur elektrodynamik bewegter körper," Rendiconti del Circolo Matematico di Palermo, vol. 28, pp. 1-28, Dec. 1909. [Online]. Available: http://www.springerlink.com/content/j8372431277v5702/

[20] M. Abraham, "Sullelettrodinamica di minkowski," Rendiconti del Circolo Matematico di Palermo, vol. 30, pp. 33-46, Dec. 1910. [Online]. Available: http://www.springerlink.com/content/h30845351ng5tv43/

[21] S. M. Barnett and R. Loudon, "The enigma of optical momentum in a medium," Philosophical Transactions of the Royal Society A: Mathematical, Physical and Engineering Sciences, vol. 368, no. 1914, pp. 927 -939, Mar. 2010. [Online]. Available: http: //rsta.royalsocietypublishing.org/content/368/1914/927.abstract

[22] S. M. Barnett, "Resolution of the Abraham-Minkowski dilemma," Physical Review Letters, vol. 104, no. 7, p. 070401, Feb. 2010. [Online]. Available: http://link.aps.org/doi/10.1103/PhysRevLett.104.070401

[23] M. Mansuripur, "Resolution of the Abraham-Minkowski controversy," Optics Communications, vol. 283, no. 10, pp. 1997-2005, May 2010. [Online]. Available: http://www.sciencedirect.com/science/article/pii/ S0030401810000131

[24] W. She, J. Yu, and R. Feng, "Observation of a push force on the end face of a nanometer silica filament exerted by outgoing light," Physical Review Letters, vol. 101, no. 24, p. 243601, Dec. 2008. [Online]. Available: http://link.aps.org/doi/10.1103/PhysRevLett.101.243601

[25] M. Mansuripur, "Comment on "Observation of a push force on the end face of a nanometer silica filament exerted by outgoing light"," Physical Review Letters, vol. 103, no. 1, p. 019301, Jul. 2009. [Online]. Available: http://link.aps.org/doi/10.1103/PhysRevLett.103.019301

[26] I. Brevik, "Comment on "Observation of a push force on the end face of a nanometer silica filament exerted by outgoing light"," Physical Review Letters, vol. 103, p. 219301, Nov. 2009. [Online]. Available: http://link.aps.org/doi/10.1103/PhysRevLett.103.219301

[27] V. Kaajakari, Practical MEMS. Las Vegas Nev.: Small Gear Pub., 2009.

[28] R. P. Feynman, "There's plenty of room at the bottom," Engineering and Science, vol. 23:5, Feb. 1960. [Online]. Available: http: //resolver.caltech.edu/CaltechES:23.5.0 
Masters Thesis - Christopher Pope McMaster University - Eng. Physics

[29] M. Madou, Fundamentals of microfabrication : the science of miniaturization, 2nd ed. Boca Raton: CRC Press, 2002. [Online]. Available: http://libcat.mcmaster.ca/index.jsp?Ntt1=1522329\&Ntk1= Bib+No.\&Ntx1=mode + matchallpartial

[30] A. M. Nicolson, "Generating and transmitting electric currents," 1918. [Online]. Available: http://www.google.com/patents?vid=2212845

[31] H. C. Nathanson and R. A. Wickstrom, "A RESONANT-GATE SILICON SURFACE TRANSISTOR WITH HIGH-Q BAND-PASS PROPERTIES," Applied Physics Letters, vol. 7, p. 84, 1965. [Online]. Available: http://link.aip.org/link/APPLAB/v7/i4/p84/s1

[32] H. C. Nathanson, W. E. Newell, R. A. Wickstrom, and J. R. Davis, "The resonant gate transistor," IEEE Transactions on Electron Devices, vol. 14, no. 3, pp. 117-133, Mar. 1967.

[33] F. Chen, J. Brotz, U. Arslan, C. Lo, T. Mukherjee, and G. K. Fedder, "CMOS-MEMS resonant RF mixer-filters," in 18th IEEE International Conference on Micro Electro Mechanical Systems, 2005. MEMS 2005. IEEE, Feb. 2005, pp. 24- 27.

[34] K. E. Petersen, "Silicon as a mechanical material," Proceedings of the IEEE, vol. 70, no. 5, pp. 420- 457, May 1982. [Online]. Available: http://dx.doi.org/10.1109/PROC.1982.12331

[35] K. E. Petersen, "Micromechanical light modulator array fabricated on silicon," Applied Physics Letters, vol. 31, p. 521, 1977. [Online]. Available: http://link.aip.org/link/APPLAB/v31/i8/p521/s1

[36] K. E. Petersen, "Silicon torsional scanning mirror," IBM Journal of Research and Development, vol. 24, no. 5, pp. 631-637, Sep. 1980.

[37] L. J. Hornbeck, "Digital light processing for high-brightness highresolution applications," in Proc. SPIE, vol. 3013. SPIE, 1997, pp. 27-40. [Online]. Available: http://focus.ti.com/download/dlpdmd/ 141_hornbeck.pdf

[38] G. Zhang and P. E. Jessop, "Microelectromechanical system based variable optical attenuator by vertically bending waveguides," in $J$. Vac. Sci. Technol. A, vol. 24. AVS, May 2006, pp. 802-806. [Online]. Available: http://link.aip.org/link/?JVA/24/802/1

[39] A. Gasparyan, H. Shea, S. Arney, V. Aksyuk, M. E. Simon, F. Pardo, H. B. Chan, J. Kim, J. Gates, J. S. Kraus, S. Goyal, D. Carr, and 
Masters Thesis - Christopher Pope McMaster University - Eng. Physics

R. Kleiman, "Drift-Free, 1000 g mechanical shock tolerant single-crystal silicon two-axis MEMS tilting mirrors in a 10001000-port optical crossconnect," in Optical Fiber Communications Conference, 2003. OFC 2003. IEEE, Mar. 2003, pp. PD36- P1-3 vol.3.

[40] H. Liu and F. Chollet, "Moving polymer waveguides and latching actuator for 2 x 2 MEMS optical switch," Journal of Microelectromechanical Systems, vol. 18, no. 3, pp. 715-724, Jun. 2009. [Online]. Available: http://ieeexplore.ieee.org/xpls/abs_all.jsp?arnumber $=4814551$

[41] J. E. Graebner, S. Pau, and P. L. Gammel, "All-optical excitation and detection of microelectrical-mechanical systems," Applied Physics Letters, vol. 81, p. 3531, 2002. [Online]. Available: http://link.aip.org/ link/APPLAB/v81/i19/p3531/s1\&Agg=doi

[42] M. L. Povinelli, M. Loncar, M. Ibanescu, E. J. Smythe, S. G. Johnson, F. Capasso, and J. D. Joannopoulos, "Evanescent-wave bonding between optical waveguides," Optics Letters, vol. 30, no. 22, pp. 3042-3044, Nov. 2005. [Online]. Available: http: //ol.osa.org/abstract.cfm?URI=ol-30-22-3042

[43] M. Li, W. H. P. Pernice, C. Xiong, T. Baehr-Jones, M. Hochberg, and H. X. Tang, "Harnessing optical forces in integrated photonic circuits," Nature, vol. 456, no. 7221, pp. 480-484, Nov. 2008. [Online]. Available: http://dx.doi.org/10.1038/nature07545

[44] M. Li, P. H. P., and T. X., "Broadband all-photonic transduction of nanocantilevers," Nat Nano, vol. 4, no. 6, pp. 377-382, Jun. 2009. [Online]. Available: http://dx.doi.org/10.1038/nnano.2009.92

[45] G. S. Wiederhecker, L. Chen, A. Gondarenko, and M. Lipson, "Controlling photonic structures using optical forces," Nature, vol. 462, no. 7273, pp. 633-636, Dec. 2009. [Online]. Available: http: //dx.doi.org/10.1038/nature08584

[46] G. Lin, R. E. Palmer, K. S. Pister, and K. P. Roos, "Miniature heart cell force transducer system implemented in MEMS technology," IEEE Transactions on Biomedical Engineering, vol. 48, no. 9, pp. 996-1006, Sep. 2001.

[47] J. B. Hartle, Gravity : an introduction to Einstein's general relativity. San Francisco: Addison-Wesley, 2003.

[48] D. Griffiths, Introduction to electrodynamics, 3rd ed. Upper Saddle River N.J.: Prentice Hall, 1999. 
[49] J. H. Poynting, "On the transfer of energy in the electromagnetic field," Philosophical Transactions of the Royal Society of London, vol. 175, pp. 343-361, Jan. 1884. [Online]. Available: http: //rstl.royalsocietypublishing.org/cgi/doi/10.1098/rstl.1884.0016

[50] I. Brevik, "Experiments in phenomenological electrodynamics and the electromagnetic energy-momentum tensor," Physics Reports, vol. 52, no. 3, pp. 133-201, May 1979. [Online]. Available: http://www. sciencedirect.com/science/article/pii/0370157379900747

[51] R. Pfeifer, T. Nieminen, N. Heckenberg, and H. Rubinsztein-Dunlop, "Colloquium: Momentum of an electromagnetic wave in dielectric media," Reviews of Modern Physics, vol. 79, pp. 1197-1216, Oct. 2007. [Online]. Available: http://link.aps.org/doi/10.1103/RevModPhys.79.1197

[52] N. L. Balazs, "The Energy-Momentum tensor of the electromagnetic field inside matter," Physical Review, vol. 91, no. 2, pp. 408-411, Jul. 1953. [Online]. Available: http://link.aps.org/doi/10.1103/PhysRev.91.408

[53] M. J. Padgett, "On diffraction within a dielectricmedium as an example of the minkowskiformulation of optical momentum," Optics Express, vol. 16, no. 25, pp. 20864-20868, Dec. 2008. [Online]. Available: http://www.opticsexpress.org/abstract.cfm?URI=oe-16-25-20864

[54] O. Reynolds, "On certain dimensional properties of matter in the gaseous state," Philosophical Transactions of the Royal Society of London, vol. 170, pp. $727-845$, Jan. 1879. [Online]. Available: http://rstl.royalsocietypublishing.org/content/170/727.short

[55] R. A. Beth, "Mechanical detection and measurement of the angular momentum of light," Physical Review, vol. 50, no. 2, p. 115, Jul. 1936. [Online]. Available: http://link.aps.org/doi/10.1103/PhysRev.50.115

[56] R. V. Jones and J. C. S. Richards, "The pressure of radiation in a refracting medium," Proceedings of the Royal Society of London. Series A. Mathematical and Physical Sciences, vol. 221, no. 1147, pp. $480-498$, Feb. 1954. [Online]. Available: http: //rspa.royalsocietypublishing.org/content/221/1147/480.abstract

[57] R. V. Jones and B. Leslie, "The measurement of optical radiation pressure in dispersive media," Proceedings of the Royal Society of London. A. Mathematical and Physical Sciences, vol. 360, no. 1702, pp. $347-363$, Apr. 1978. [Online]. Available: http: ///rspa.royalsocietypublishing.org/content/360/1702/347.abstract 
[58] A. Ashkin and J. M. Dziedzic, "Radiation pressure on a free liquid surface," Physical Review Letters, vol. 30, no. 4, pp. 139-142, Jan. 1973. [Online]. Available: http://link.aps.org/doi/10.1103/PhysRevLett.30.139

[59] M. Liu, T. Zentgraf, Y. Liu, G. Bartal, and X. Zhang, "Light-driven nanoscale plasmonic motors," Nat Nano, vol. 5, no. 8, pp. 570-573, 2010. [Online]. Available: http://dx.doi.org/10.1038/nnano.2010.128

[60] L. D. Landau and E. M. Lifshits, Theory of elasticity, 2nd ed. Oxford; New York: Pergamon Press, 1970.

[61] M. A. Hopcroft, W. D. Nix, and T. W. Kenny, "What is the young's modulus of silicon?" Journal of Microelectromechanical Systems, vol. 19, no. 2, pp. 229-238, Apr. 2010. [Online]. Available: http://dx.doi.org/10.1109/JMEMS.2009.2039697

[62] W. Weaver, S. P. Timoshenko, and D. H. Young, Vibration Problems in Engineering, 5th ed. Wiley-Interscience, Jan. 1990.

[63] N. Ida, Engineering electromagnetics, 2nd ed. New York: Springer, 2003.

[64] G. T. Reed and A. P. Knights, Silicon photonics : an introduction. Chichester; Hoboken, NJ: John Wiley, 2004.

[65] R. Serway and J. W. Jewett, Physics for scientists and engineers., 6th ed. Belmont CA: Thomson-Brooks/Cole, 2004.

[66] R. Soref, J. Schmidtchen, and K. Petermann, "Large single-mode rib waveguides in GeSi-Si and Si-on-SiO2," Quantum Electronics, IEEE Journal of, vol. 27, no. 8, pp. 1971-1974, 1991.

[67] S. Pogossian, L. Vescan, and A. Vonsovici, "The single-mode condition for semiconductor rib waveguides with large cross section," Lightwave Technology, Journal of, vol. 16, no. 10, pp. 1851-1853, 1998.

[68] G. Fowles and G. Cassiday, Analytical mechanics, 7th ed. Pacific Grove Calif.; London: Brooks/Cole, 2005.

[69] S. Campbell, The science and engineering of microelectronic fabrication. New York: Oxford University Press, 1996.

[70] K. Williams and R. Muller, "Etch rates for micromachining processing," Microelectromechanical Systems, Journal of, vol. 5, no. 4, pp. 256-269, 1996. [Online]. Available: http://dx.doi.org/10.1109/84.546406

[71] Google Inc., Google Sketchup. [Online]. Available: http://sketchup. google.com/ 
Masters Thesis - Christopher Pope McMaster University - Eng. Physics

[72] E. Ollier, "Optical MEMS devices based on moving waveguides," IEEE Journal of Selected Topics in Quantum Electronics, vol. 8, no. 1, pp. 155162, Feb. 2002.

[73] E. Huante-Ceron, "SOI ridge waveguide processing," McMaster University, Unpublished Guide, 2009.

[74] H. Seidel, L. Csepregi, A. Heuberger, and H. Baumgartel, "Anisotropic etching of crystalline silicon in alkaline solutions," Journal of The Electrochemical Society, vol. 137, no. 11, pp. 3612-3626, Nov. 1990. [Online]. Available: http://link.aip.org/link/?JES/137/3612/1

[75] Y. Uenishi, M. Tsugai, and M. Mehregany, "Micro-opto-mechanical devices fabricated by anisotropic etching of (110) silicon," Journal of Micromechanics and Microengineering, vol. 5, pp. 305-312, Dec. 1995. [Online]. Available: http://iopscience.iop.org/0960-1317/5/4/007

[76] D. Taillaert, P. Bienstman, and R. Baets, "Compact efficient broadband grating coupler for silicon-on-insulator waveguides," Optics Letters, vol. 29, no. 23, pp. 2749-2751, Dec. 2004. [Online]. Available: http://ol.osa.org/abstract.cfm?URI=ol-29-23-2749

[77] D. R. Lide, Ed., CRC Handbook of Chemistry and Physics, 92th Edition (Internet Version 2011), 92nd ed. Boca Raton, FL: CRC Press/Taylor and Francis, 2011. [Online]. Available: http://www.hbcpnetbase.com

[78] A. Cowen, G. Hames, D. Monk, S. Wilcenski, and B. Hardy, "SOIMUMPs design handbook," MEMSCAP Inc., Tech. Rep., 2011, revison 8.0. [Online]. Available: http://www.memscap.com/products/ mumps/soimumps/reference-material

[79] A. Rampal, "Experimental and theoretical study of quartz and InP piezoelectric MEMS resonators," Master's Thesis, McMaster University, Hamilton, Ontario, 2009.

[80] J. Wei, C. Yue, Z. L. Chen, Z. W. Liu, and P. M. Sarro, "A silicon MEMS structure for characterization of femto-farad-level capacitive sensors with lock-in architecture," Journal of Micromechanics and Microengineering, vol. 20, no. 6, p. 064019, Jun. 2010. [Online]. Available: http://iopscience.iop.org/0960-1317/20/6/064019

[81] CadSoft Computer, EagleCAD. [Online]. Available: http://www. cadsoftusa.com/ 
[82] J. Ardizzoni, "A practical guide to High-Speed Printed-Circuit-Board layout," Analog Dialog, vol. 39, no. 3, Sep. 2005. [Online]. Available: http: //www.analog.com/library/analogDialogue/archives/39-09/layout.html

[83] S. Guinta, "Ask the applications engineer - 21: Capacitance and capacitors," Analog Dialog, vol. 30, no. 2, 1996. [Online]. Available: http://www.analog.com/library/analogDialogue/Anniversary/21.html

[84] Andeed-Hagerling Inc, 2500A $1 \mathrm{kHz}$ Ultra-Precision Capacitance Bridge Manual, Cleveland, Ohio.

[85] Amptek Inc., A250 Charge Sensitive Preamplifier Datasheet, Bedford, MA. [Online]. Available: http://www.amptek.com/a250.html

[86] Stanford Research Systems, Model SR844 RF Lock-In Amplifier User's Manual, Sunnyvale, California, 2007. [Online]. Available: http://www.thinksrs.com/downloads/PDFs/Manuals/SR844m.pdf

[87] Stanford Research Systems, "About Lock-In amplifiers," Application Note. [Online]. Available: http://www.thinksrs.com/support/app.htm

[88] National Instruments Corporation, LabVIEW 8.2. [Online]. Available: http://www.ni.com/labview/ 